\title{
Taxonomic revision of Amorimia W.R. Anderson (Malpighiaceae)
}

\author{
- 0 Rafael Felipe de Almeida ${ }^{1,2,3}$
}

Received: 4.8.2017; accepted: 2.04 .2018

\begin{abstract}
Taxonomic revision of Amorimia W.R. Anderson (Malpighiaceae)). The taxonomic revision of Amorimia (Malpighiaceae) is presented, including typifications, and descriptions for all accepted species. The genus is endemic to Seasonally Dry Tropical Forests and Rainforests of South America, and its species can be distinguished by morphological details of leaves, indumenta, inflorescences, flowers, and fruits. This study includes an identification key for the subgenera and species of Amorimia, illustrations, distribution maps, conservation risk assessments, and comments on ecology, nomenclature, and taxonomy for all species. Additionally, I provide a key to differentiate Amorimia from the remaining genera of the Malpighioid clade.
\end{abstract}

Keywords: Malpighiales, Mascagnia, Neotropical flora, Seasonally Dry Tropical Forests, Taxonomy

RESUMO - (Revisão taxonômica de Amorimia W.R. Anderson (Malpighiaceae)). Apresento a revisão taxonômica de Amorimia (Malpighiaceae), incluindo tipificações e descrições para todas as espécies aceitas. O gênero é endêmico a Florestas Sazonais e Úmidas da América do Sul, e suas espécies podem ser distinguidas por detalhes da morfologia de folhas, indumento, inflorescências, flores e frutos. Este estudo inclui descrições morfológicas completas para todas as espécies, além de uma chave de identificação para seus subgêneros e espécies, ilustrações, mapas de distribuição, avaliações de risco de extinção e comentários sobre ecologia, nomenclatura e taxonomia de suas espécies. Ainda, apresento uma chave de identificação para diferenciar Amorimia dos demais gêneros do clado Malpighioide.

Palavras-chave: Flora Neotropical, Florestas Tropicais Sazonalmente Secas, Malpighiales, Mascagnia, Taxonomia

\section{Introduction}

Amorimia W.R. Anderson is a small genus of Neotropical lianas, comprising 15 species distributed throughout Seasonally Dry Tropical Forests (SDTF) and Rainforests in South America, ranging from Northern Colombia to Southern Brazil and Argentina (Anderson 2006, BFG 2015, Almeida et al. 2016a, 2016b, 2017a, 2017b). The genus is monophyletic and currently placed in a basal grade in the Malpighioid clade (sensu Almeida et al. 2017b), alongside Ectopopterys W.R. Anderson, Mascagnia (Bertero ex DC.) Bertero s.s., Calcicola W.R. Anderson \& C.C. Davis, Malpighia L., and several paleotropical lineages (i.e., Aspidopterys A. Juss., Caucanthus Forssk., Diaspis Nied., Digoniopterys Arènes, Madagasikaria C.C. Davis, Rhynchophora Arènes, Microsteira Baker, and Triaspis Burch.) (Davis \&
Anderson 2010, Almeida et al. 2017b). Amorimia is currently recognized by synapomorphies such as the presence of extra-floral nectaries on the abaxial side of bracts and bracteoles, petals pubescent on both sides, samaras with two free lateral wings larger than the dorsal wing, and the production of monofluoroacetate (Anderson 2006, Almeida et al. 2017b).

After extensive field and herbarium studies, in association with additional molecular studies (Almeida et al. 2017b), I presented new morphological, evolutionary and biogeographical data to the systematics of Amorimia (Almeida 2017). These systematic efforts paved the way for the elaboration of a new, comprehensive and updated taxonomic revision for the genus, herein presented. This study includes full morphological descriptions for all taxa, along with an identification key for its subgenera and species, illustrations, distribution maps, conservation risk

1. Universidade Estadual de Feira de Santana, Departamento de Ciências Biológicas, Programa de Pós-Graduação em Botânica, Av. Transnordestina s/n, Novo Horizonte, 44036-900 Feira de Santana, BA, Brasil

2. Universidade Federal de Minas Gerais, Instituto de Ciências Biológicas, Departamento de Botânica, Programa de Pós-Graduação em Biologia Vegetal, Av. Antonio Carlos, 6627, Pampulha, 31270-901 Belo Horizonte, MG, Brasil

3. Corresponding author: dealmeida.rafaelfelipe@gmail.com 
assessments, and comments on ecology, nomenclature, and taxonomy. Additionally, I provide a key to differentiate Amorimia from the remaining genera of the Malpighioid clade.

\section{Material and methods}

Morphological descriptions and phenology of the studied species were based on herbarium specimens (ALCB, AMAZ, ASE, BAH, BM, BHCB, BOTU, CEN, CEPEC, CESJ, CGMS, COL, CPAP, CVRD, CTES, CUZ, EAC, ESA, F, FLOR, FUEL, FURB, FZB, G, GH, GUA, HAS, HB, HCF, HEPH, HISA, HRB, HRCB, HSJRP, HST, HUCP, HUCS, HUEFS, HUEM, HUESC, HUFG, HUFU, HUPG, HURB, HUT, HUVA, IAC, IAN, ICN, INPA, IPA, JPB, L, LIL, K, MAC, MBM, MBML, MG, MICH, MO, MOSS, MPU, NY, OUPR, P, PACA, PAMG, PEUFR, PMSP, R, RB, RBR, RFA, S, SI, SMF, SP, SPF, SPSF, TEPB, U, UB, UEC, UFP, UFMS, UFMT, UFRN, UPCB, US, USZ, W, VIC, and VIES), and spirit specimens [herbaria acronyms according to Thiers (2017), continuously updated]. The indumenta terminology follows Anderson (1981), structure shapes follow Radford et al. (1974), the inflorescence terminology and morphology follow Weberling (1965, 1989), and fruit terminology follows Spjut (1994) and Anderson (1981). The conservation status was proposed following the recommendations of IUCN Red List Categories and Criteria, Version 3.1 (IUCN 2012). GeoCAT (Bachman et al. 2011) was used for calculating the Extent of Occurrence (EOO) and the Area of Occupancy (AOO). Maps were elaborated using ArcGIS 9.3 software (ESRI 2010), geographical coordinates were obtained from herbaria specimens and fieldwork data, and shapefiles were obtained from Sarkinen et al. (2011) and WWF (2017).

\section{Results and Discussion}

In general, most botanists still have a lot of difficulty differentiating the genera segregated from Mascagnia s.l. This can be evidenced by the great number of specimens from different genera erroneously assigned to Mascagnia in most from the 93 visited herbaria (pers. obs.). Similarities and differences between these various genera are presented in Anderson (2006) and partially in Almeida et al. (2016a). Amorimia is placed in the Malpighioid clade, a group combining all genera from the Malpighia clade + Amorimia and Ectopopterys (figure 1, Almeida et al. 2017b). The Malpighioid clade is macromorphologically diverse, but anatomical studies (Almeida et al. 2017a, Mello 2017) indicate that this group is micromorphologically supported. A key to distinguish the genera from the Malpighioid clade is presented below.

Key to the genera of the Malpighioid clade

1. Flowers actinomorphic, lacking sepal glands, stigmas terminal; Paleotropics

2. Styles truncate at apex

3. Flower buds smooth, petals with entire margins, reflexed; Asia .................Aspidopterys (figure1 k-1)

3. Flower buds keeled, petals with fimbriate to undulate margins, patent; Africa and Arabian Peninsula

4. Leaves spirally-alternate; petals with fimbriate margins, anthers with thecae smooth, 2-carpelate.... Diaspis

4. Leaves opposite; petals with undulate margins, anthers with thecae rugose, 3-carpelate ... Caucanthus

2. Styles with a projection (lobe) at apex

5. Plants monoecious; flower buds keeled, petals with fimbriate margins, style 1-lobed at apex;

Africa Triaspis (figure1 m-n)

5. Plants dioecious; flower buds smooth, petals with entire margins, style 2-lobed at apex (sometimes reduced to a triangular lobe); Madagascar

6. Leaves up to $5 \mathrm{~mm}$ wide; petals narrowly spatulate and densely sericeous over whole abaxial surface, styles longly-lobed Digoniopterys

6. Leaves at least $1 \mathrm{~cm}$ wide, mostly much wider; petals elliptical to orbicular, glabrous or thinly sericeous on abaxial keel, styles shortly-lobed

7. Stipules enlarged, leaf-like, persistent; flowers born in elongated axillary thyrsi of 8 or more 1-flowered cincinni, with elongated internodes .............. Madagasikaria (figure $1 \mathrm{q}-\mathrm{r}$ )

7. Stipules reduced, triangular, persistent to deciduous; flowers born in umbels or corymbs of 4-many cincinni, with inconspicuous internodes 
8. Ovary bearing initials for lateral wings and dorsal crest on each carpel, visible even in young flowers Microsteira (figure $1 \mathrm{~s}-\mathrm{t}$ )

8. Ovary lacking any obvious sign of initials for wings or crests Rhynchophora (figure 1 o-p)

1. Flowers zygomorphic, bearing sepal glands, stigmas lateral; Neotropics

9. Connectives of anthers bearing large glands, styles foliate at apex, dorsal wing of samaras larger than the laterals Ectopopterys (figure $1 \mathrm{a}-\mathrm{b})$

9. Connectives of anthers bearing an inconspicuous glandular tissue, styles truncate at apex, lateral wings of samaras larger than the dorsal

10. Mericarps indehiscent, fleshy, dorsal and lateral wings much reduced, free, fleshy at maturity

Malpighia (figure $1 \mathrm{i}-\mathrm{j}$ )

10. Mericarps dehiscent, dry, lateral wings larger, fused, dry

11. Bracts glandular, bracteoles 2-6-glandular; petals adaxially pubescent, lateral wings of samaras free Amorimia (figure $1 \mathrm{c}-\mathrm{d}$ )

11. Bracts eglandular, bracteoles $0-1$-glandular; petals adaxially glabrous, lateral wings of samaras fused

12. Lianas to scandent shrubs; leaves long-petiolate; thyrsi to corymbs; flower buds keeled Mascagnia (figure 1 e-f) 12. Erect shrubs; leaves subsessile; 2-4-flowered umbels; flower buds smooth ........ Calcicola (figure $1 \mathrm{~g}-\mathrm{h}$ )

\section{Taxonomic history}

Many species currently treated under Amorimia were previously treated by many authors as part of a much wider and polyphyletic Hiraea Jacq., or of an also polyphyletic Mascagnia. Based on recent systematic and taxonomic studies, we now know that these two artificial assemblages actually represent eight independent lineages (Anderson 2006, Davis \& Anderson 2010). Jussieu (1833) described under Hiraea the first two species of Amorimia. H. pubiflora A. Juss. and $H$. rigida A. Juss. Grisebach (1858) transferred these species to Mascagnia, a genus based on Hiraea sect. Mascagnia DC. Previously, Mascagnia included a single accepted species, Hiraea macradena DC., and the genus was only brought to light by Grisebach's monograph on Brazilian Malpighiaceae (1858). Mascagnia sensu Grisebach (1858) was characterized by the presence of samaras with two lateral wings, and pedicels articulate at mid length, while Hiraea was distinguished by its samaras with 1-2 lateral wings, and pedicels proximally articulate. The sections proposed by Grisebach (1858) for Mascagnia were based on the shape and size of lateral wings of the samaras, with M. pubiflora (A. Juss.) Griseb. and $M$. rigida (A. Juss.) Griseb. being placed in $M$. sect. Pleuropterys Griseb. This section was characterized by the samaras with distinct lateral wings, and a single and shortened (sometimes absent) dorsal wing. In Grisebach's monograph (1858), he proposed new combinations and taxonomic novelties for this section, which at the time comprised nine accepted names: Mascagnia biglandulosa (A. Juss.) Griseb., M. bunchosioides (A. Juss.) Griseb., M. coriacea Griseb., M. doniana Griseb., M. exotropica Griseb., M. fluminensis (Griseb.) Griseb., M. psilophylla (A. Juss.) Griseb., M. pubiflora, and M. rigida.

Niedenzu (1914) considered M. biglandulosa and M. bunchosioides as synonyms of Heladena multiflora (Hook. \& Arn.) Nied. The author also presented taxonomic novelties for this section, such as the description of $M$. amazonica Nied., and $M$. lehmanniana Nied. Few years later, Morton (1932) described a new species of $M$. sect. Pleuropterys endemic to Colombia, M. dumetorum C.V. Morton. However, Morton later noticed that his name was illegitimate, since it was a later homonym of $M$. dumetorum Griseb., and proposed M. concinna C.V. Morton as a replacement name. Johnson (1986) transferred M. psilophylla to Callaeum Small, while Anderson (1987) transferred M. fluminensis to Heteropterys Kunth, and Anderson and Davis (2007) transferred M. lehmanniana also to Heteropterys. Recently, aiming to finally render Mascagnia monophyletic, Anderson (2006) described Amorimia as a new genus, transferring all species from $M$. sect. Pleuropterys, based on morphological and molecular data (Cameron et al. 2001, Davis et al. 2001, 2002, Anderson 2006).

On the other hand, the broad concept of Amorimia rigida (A. Juss.) W.R. Anderson adopted by previous authors led to a misunderstanding of the morphological variation within this species distribution range 


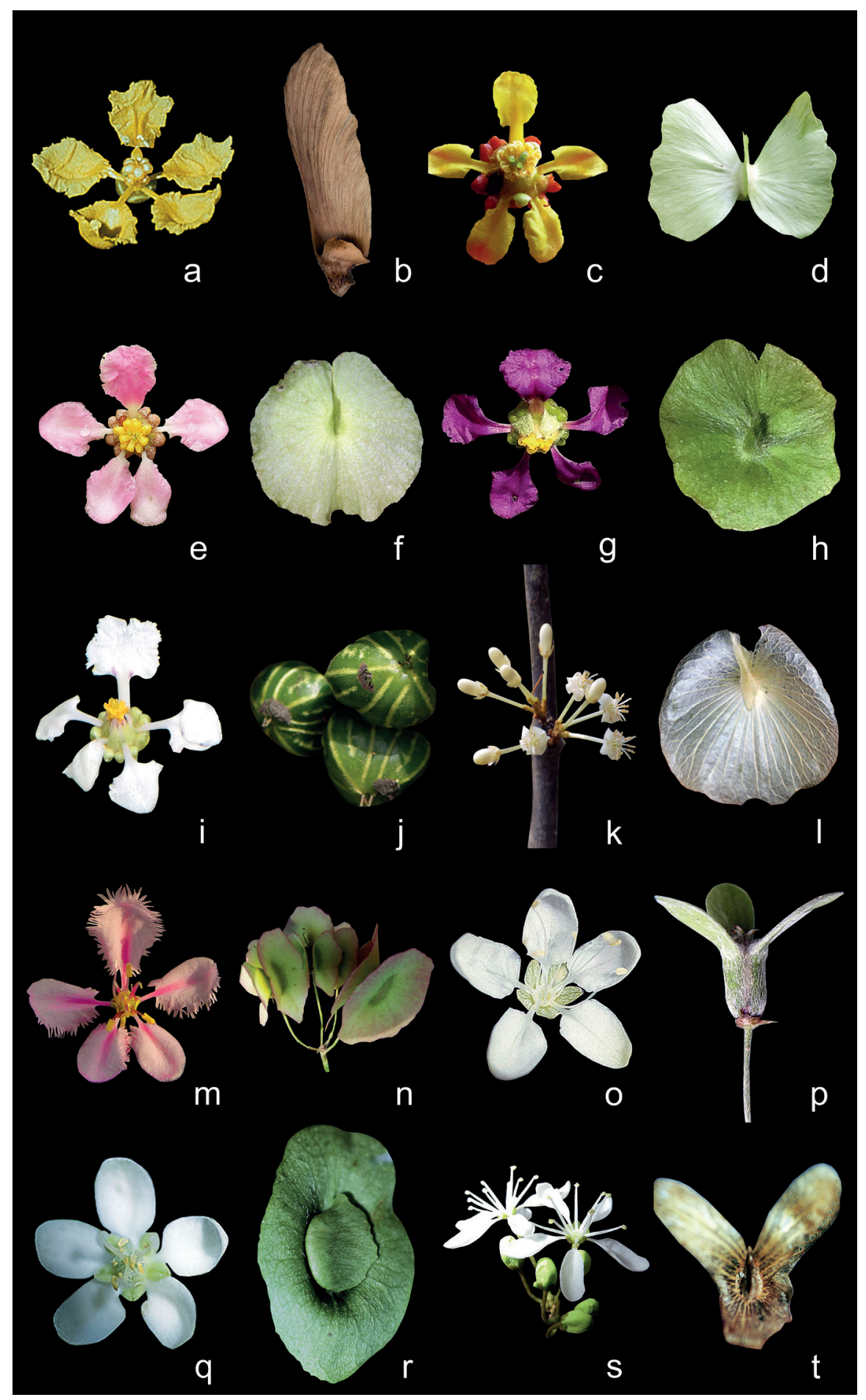

Figure 1. Morphological variation of flowers and fruits within the genera of the Malpighioid clade. a. flower in frontal view of Ectopopterys soejartoi. b. samara in side view of Ectopopterys soejartoi. c. flower in frontal view of Amorimia maritima. d. samara in dorsal view of Amorimia maritima. e. flower in frontal view of Mascagnia cordifolia. f. samara in dorsal view of Mascagnia sepium. g. flower in frontal view of Calcicola sericea. h. samara in dorsal view of Calcicola sericea. i. flower in frontal view of Malpighia fucata. j. drupes in dorsal view of Malpighia fucata. $\mathrm{k}$. flower in frontal view of Aspidopterys canariensis. 1. samara in dorsal view of Aspidopterys concava. m. flower in frontal view of Triaspis glaucophylla. n. samara in dorsal view of Triaspis glaucophylla. o. flower in frontal view of Rhynchophora phillipsonii. p. samaras in side view of Rhynchophora phillipsonii. q. flower in frontal view of Madagasikaria andersonii. r. samara in dorsal view of Madagasikaria andersonii. s. flower in side view of Microsteira pluriseta. t. samara in dorsal view of Microsteira sp. (photographs a-b by D.D. Soejarto, c-d by F. Flores, e by R. Sartin, f by M.O.O. Pellegrini, g-h by M.R. Pace, i-j by P. Acevedo-Rodríguez, k by D. Valke, 1 by L. Pok, m by B. Pilenaar, $n$ by L. Ann, o-r and t by C.C. Davis, s. by L. Nusbaumer). 
(Anderson 2006, Almeida et al. 2016b). Consequently, 10 species have been recognized in the $A$. rigida complex in the past few years: A. andersonii R.F. Almeida, A. camporum W.R. Anderson, A. candidae R.F. Almeida, $A$. coriacea (Griseb.) R.F. Almeida, $A$. kariniana W.R. Anderson, A. pellegrinii R.F. Almeida, A. rigida s.s., A. septentrionalis W.R. Anderson, A. tumida R.F. Almeida \& A.C. Marques, and A. velutina W.R. Anderson (Anderson 2006, Almeida et al. 2016b, Almeida et al. 2017a) (figure 2).

\section{Conservation}

Amorimia has been the subject of countless toxicology studies in veterinary medicine since the 1960's in Brazil (Tokarnia et al. 1961, Tokarnia \& Döbereiner 1973). As a result, a couple of species (e.g., A. rigida and A. maritima; commonly referred either as $A$. rigida or $A$. aff. rigida) have been usually considered as responsible for bovine sudden death in different parts of the country (Tokarnia et al. 1961, 1990, 1994, 1996, 2000, 2002, Tokarnia \& Döbereiner 1973, 1985, Medeiros et al. 2002, Silva et al. 2006, 2008, Barbosa et al. 2008, Melo et al. 2008, Vasconcelos et al. 2008, Assis et al. 2009, 2010, Garcez et al. 2009, Lago et al. 2009, Borboleta et al. 2011, 2012, Nogueira et al. 2011, Pavarini et al. 2011, Schons et al. 2011, Soares et al. 2011, Lee et al. 2012, Becker et al. 2013, Duarte et al. 2013, Neto et al. 2013, Pessoa et al. 2013, Barros \& Meneguetti 2014, Arruda

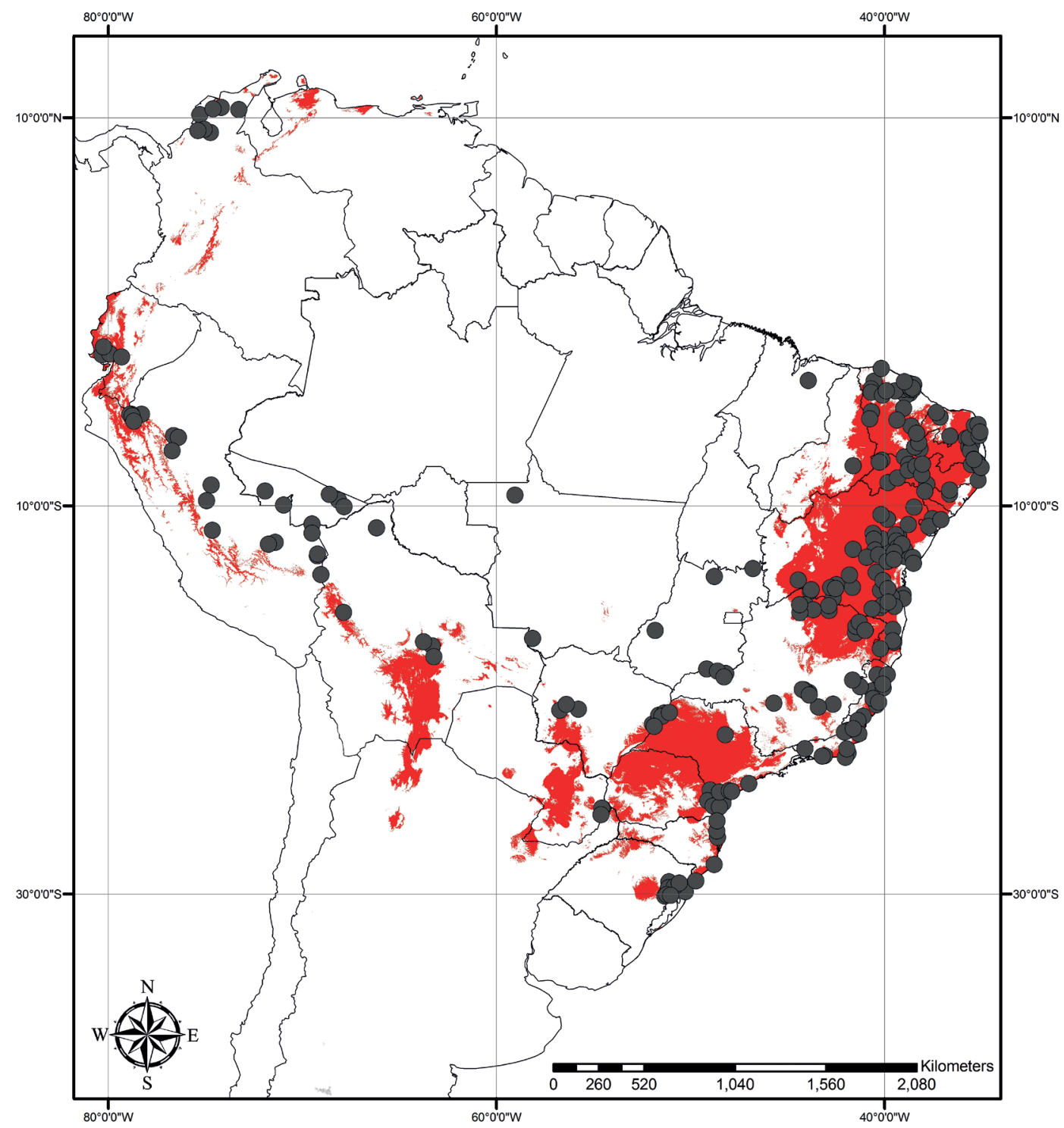

Figure 2. Amorimia distribution in Seasonally Dry Tropical Forests (red) of South America. 

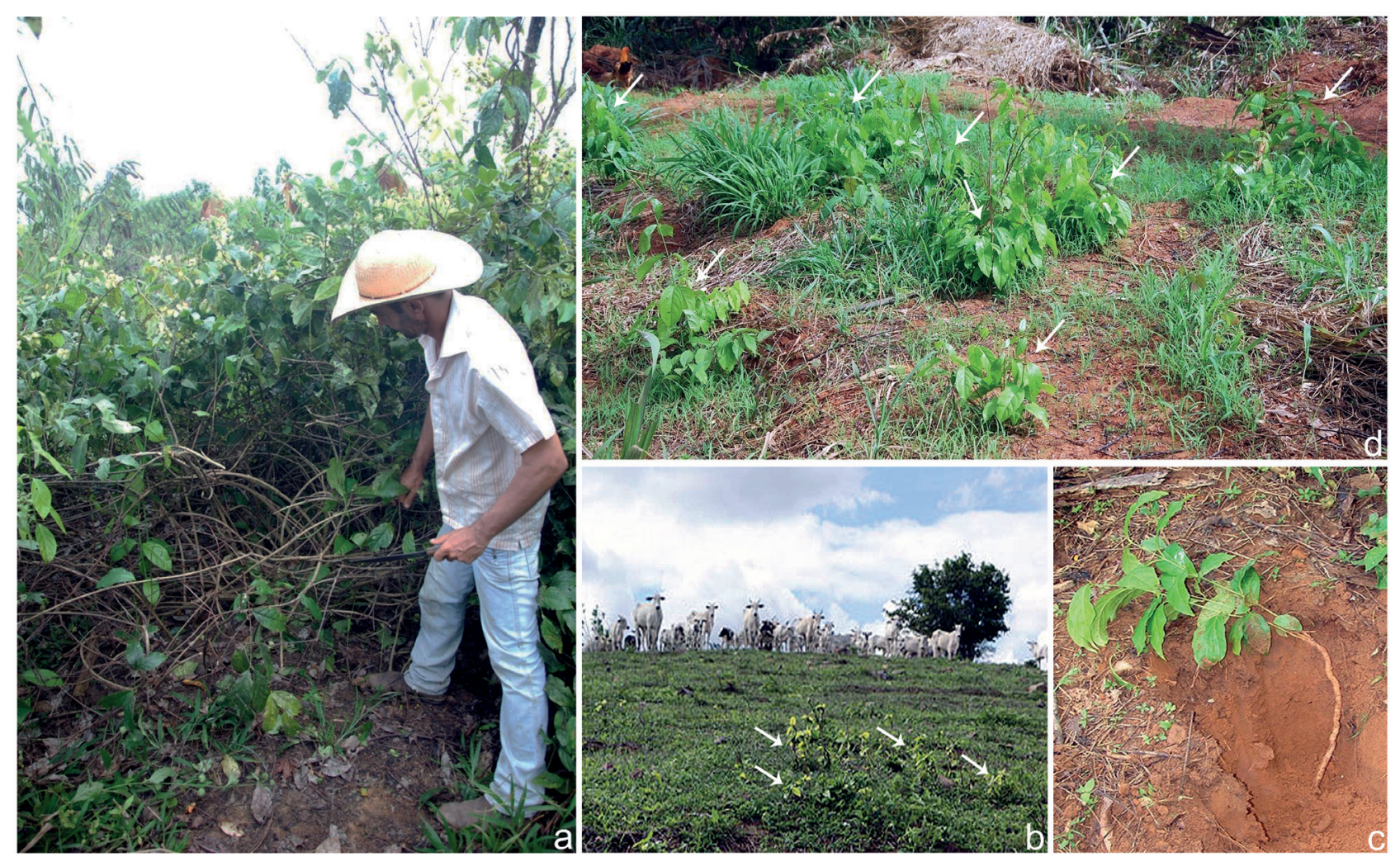

Figure 3. Natural populations of Amorimia pubiflora in pastures of Central Brazil. a. lianescent habit of an individual growing over a fence. b. resprouts growing on a pasture near cattle. c. stolons. d. closer image of resprouts of the same individual growings near each other due to its stolons (photographs by E. Moletta).

et al. 2017). Recently, broad phytochemical studies have identified monofluoracetate as the compound responsible for Amorimia's toxicity to mammals (Lee et al. 2012). In the past 60 years, some methods for controlling Amorimia outbreaks became widespread among Brazilian farmers and veterinarians, such as biological, manual, and mechanical control (Duarte et al. 2013, Pessoa et al. 2013, figure 3). Since most species of Amorimia commonly occur associated with gallery forests and waterstreams (figure 4), I believe that deforestation, associated with the increase of removal of mature individuals by farmers, has drastically affected populations of Amorimia in the past six decades. Evidence to corroborate my hypothesis resides in herbarium collections, in which most specimens of Amorimia were collected between mid 1980's and late 1990's (speciesLink 2017). Additionally, during fieldwork in Eastern Brazil, I could notice a significant number of localities known to harbor species of the genus turned into rice crops, damns, wind farms, but more commonly turned into pastures (pers. obs., figure 4).

Even though most species of Amorimia show a wide distribution range, most of them fall within the endangered conservation status, if the AOO is taken into account (e.g., A. amazonica, A. exotropica, A. maritima, A. pubiflora, A. rigida, A. septentrionalis, and $A$. velutina). It is widely known that tropical environments are highly threatened due to human activities (Myers et al. 2000, Marchese 2015). However, when considering plants regarded as harmful to livestock economy, the level of threat is significantly higher. In Brazil, is estimated that ca. $5 \%$ of the cattle and horse population annually dies from different causes, and about $15 \%$ of this total is caused by plant poisoning (Pessoa et al. 2013). Few studies regarding the natural abundance of toxic weeds in tropical environments are available in the literature (Carvalho \& Pitelli 1992). However, based on the information available, it is possible to assume that Amorimia populations are sparsely distributed within natural habitats (Carvalho \& Pitelli 1992). I confirmed this assumption during field trips to recollect species of Amorimia in central and eastern Brazil, where large populations were only found in wellpreserved gallery forests.

\section{Morphology}

Indumentum - The indumenta in Amorimia predominantly consist of $\mathrm{V}$ and $\mathrm{T}$-shaped hairs densely 

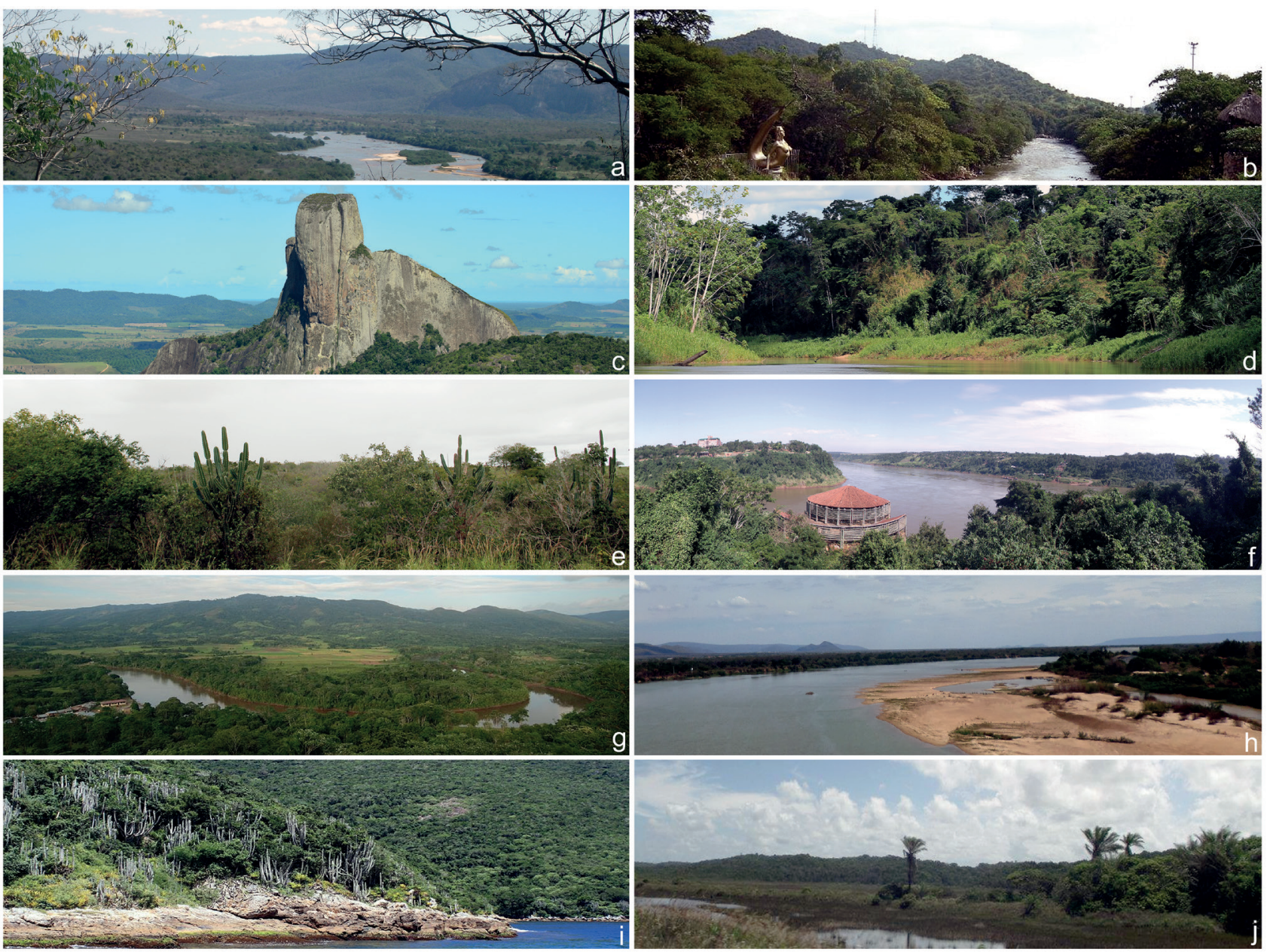

Figure 4. Several habitats occupied by species of Amorimia in South America. a. dry forests from the Jequitinhonha River, Minas Gerais, Brazil. b. dry forests from the Valledupar valley, Colombia. c. seasonally dry forests from Itamarajú, Bahia, Brazil. d. rainforests from Purus river basin, Acre, Brazil. e. dry forests from Itaberaba, Bahia, Brazil. f. seasonally dry forests from Paraná river, triple borders of Argentina, Brazil, and Paraguay. g. seasonally dry forests of Mayo river, Peru. h. dry forests of São Francisco river, Bahia, Brazil. i. dry forests from costal plains in Cabo Frio, Rio de Janeiro, Brazil. j. rainforests from Ilhéus, Bahia, Brazil (photographs a, e, f, h, j by R.F. Almeida, b by G. Barros, c by A.M.A. Amorim, d by H. Medeiros, g by A.R. Silva, i by M.O.O. Pellegrini).

to sparsely intertwined (figure 5). All species bear densely haired young stems, leaves, inflorescences, and fruits that mostly become glabrescent to completely glabrous at age. The only species that consistently show hairy leaves throughout their life span are A. concinna and A. pubiflora. Due to its mixed nature, it is quite difficult sometimes to classify the indumentum of Amorimia in a single category. Thus, in order to make it easier for general botanists, I adopted only three types of indumenta in Amorimia. tomentose, sericeous-velutine and velutine. The first type includes any indumentum made of mostly T-shaped hairs, the second by a similar proportion of V, Y and T-shaped hairs, and the latter by $\mathrm{Y}$ and $\mathrm{V}$-shaped hairs (figure 5).

Stipules - All species of Amorimia show interpetiolar stipules (figure $6 \mathrm{~b}$ ), instead of epipetiolar stipules as stated by Almeida et al. (2016a). In fact, the stipules might move during inflorescence development and end up placed at the base of leaves associated to the inflorescence and/or bracts (figure 6a). The shape of stipules seems to be a conserved character among the subgenera of Amorimia. In Amorimia subg. Amorimia stipules are triangular and in Amorimia subg. Uncinae they are narrowly-triangular (figure 6).

Leaves - The leaves in Amorimia vary in shape from elliptic to obovate, with bases ranging from cuneate to rounded or cordate, and apices ranging from rounded to acute or mucronate (figures 7-8). It is also quite common that the leaves associated with the inflorescences become wide-elliptic to orbicular, bearing mucronate to emarginate apices (figures 7-8). 

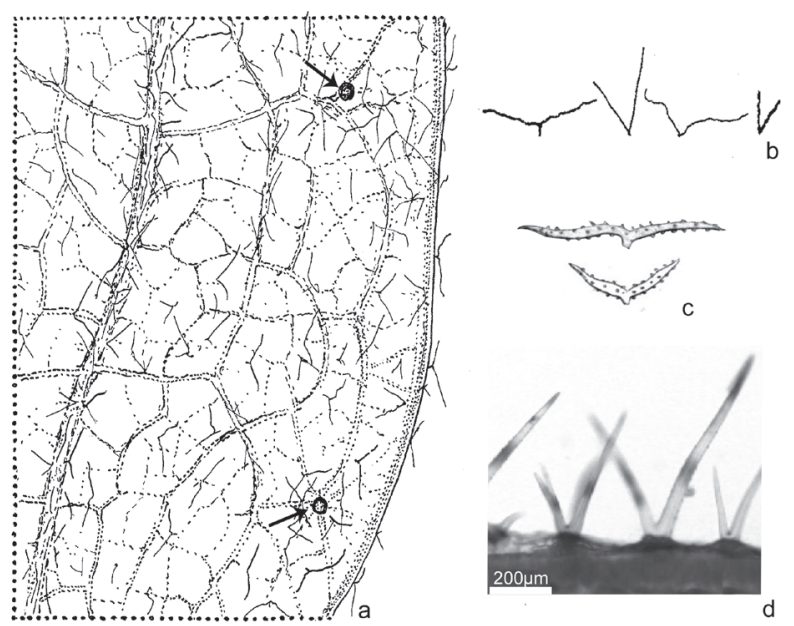

Figure 5. Indumentum and hair types in Amorimia. a. sericeousvelutine indumentum of $A$. kariniana, evidencing $\mathrm{T}$ and $\mathrm{V}$-shaped hairs. b. different shapes of $\mathrm{T}$ and $\mathrm{V}$ hairs. c. unicellular hairs with rugae in their cell walls. d. side view of V-shaped hairs (images a-b by Karin Weishaar Douthit, c by Grisebach 1858, d by A.C. Marques).

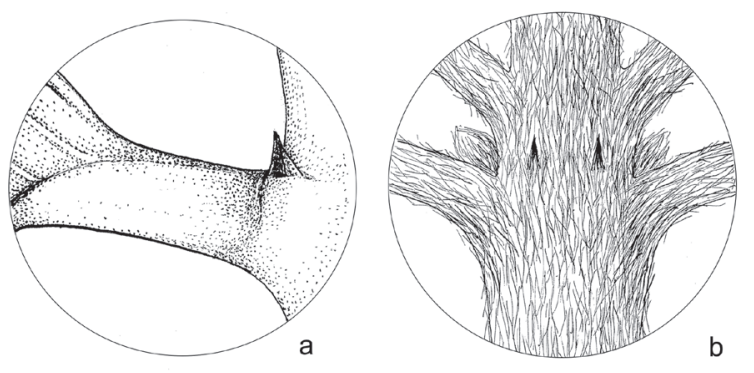

Figure 6. Position and shape of stipules in Amorimia. a. triangular, epipetiolar stipule. b. narrow-triangular, intrapetiolar stipules (drawings by Karin Weishaar Douthit).

Inflorescences - The inflorescence in Amorimia follows the general pattern of Malpighiaceae, being composed of 1-flowered cincinni arranged in a thyrse or a panicle (figure 9). When comparing its inflorescence architecture with the remaining genera of the Malpighioid clade, a reductionary pattern is

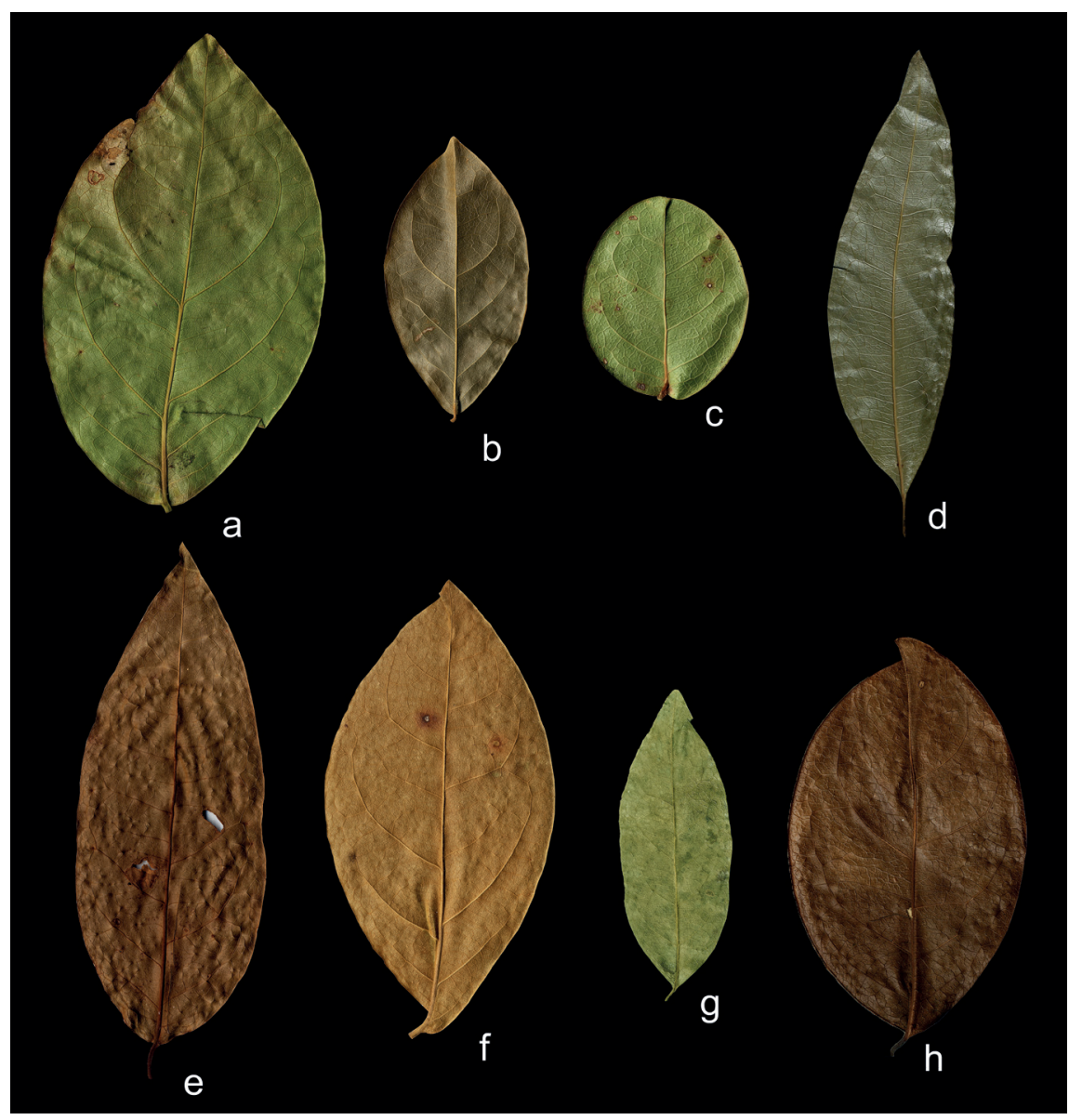

Figure 7. Leaf shapes in Amorimia subg. Amorimia. a. Amorimia rigida (R.F. Almeida 561, HUEFS). b. Amorimia pellegrinii (R.F. Almeida 614, HUEFS). c. Amorimia candidae (R.F. Almeida 594, HUEFS). d. Amorimia andersonii (L.C. Marinho 654, CEPEC). e. Amorimia exotropica (R.F. Almeida 549, HUEFS). f. Amorimia velutina (Shepherd 4409, UEC). g. Amorimia coriacea (R.F. Almeida 615, HUEFS). h. Amorimia maritima (P. Salzmann s.n., G). 


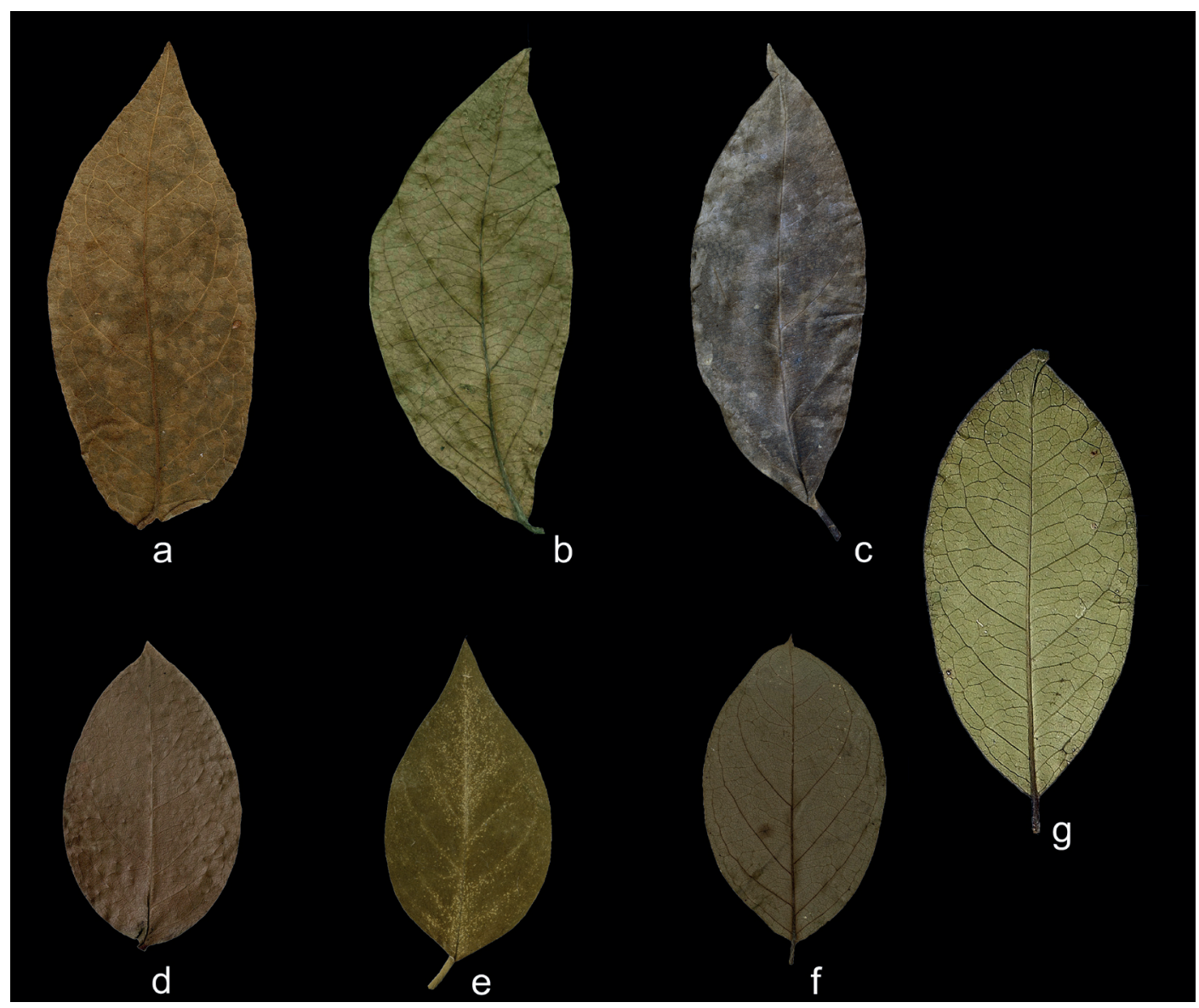

Figure 8. Leaf shapes in Amorimia subg. Uncinae. a. Amorimia pubiflora (A. Pott 10411, CGMS). b. Amorimia septentrionalis (R.F. Almeida 800, HUEFS). c. Amorimia amazonica (P. Ule 9478, MG). d. Amorimia camporum (Campos 3266, NY). e. Amorimia concinna (Castaneda 9292, NY). f. Amorimia kariniana (Haught 3070, NY). g. Amorimia tumida (M.N. Coelho 2794, HUEFS).

observed. Ectopopterys is the first lineage to diverge in this clade, showing few 1-flowered cincinni arranged in umbels, corymbs or thyrsi. Amorimia is the second lineage to diverge, showing (10-)30-40(-50) 1-flowered cincinni arranged in a thyrse, rarely in a panicle. Mascagnia and the remaining genera of the Malpighioid clade show 1-flowered cincinni, either arranged in a thyrse with reduced internodes, a corymb, or completely reduced to an umbel in Malpighia.

The 1-flowered cincinnus is divided into a peduncle, which always bears a bract at base and two bracteoles at or near the apex, and a pedicel that bears the flower (figure 9). The most defining character that distinguishes Amorimia from the remaining Malpighiaceae genera is its glandular bracts (figure 9). Its bracteoles bear (0-)1-3-pairs of glands. Nonetheless, this character is not exclusive to Amorimia, also occuring in Tetrapterys p.p.

Sepals - The sepals in Amorimia vary in pubescence, shape and posture of the apex (figure 10). The abaxial side of sepals are pubescent in Amorimia subg. Amorimia, and glabrous in Amorimia subg. Uncinae (figure 10). The apex of sepals is rounded in Amorimia subg. Amorimia, and acute in Amorimia subg. Uncinae (figure 10). Aditionally, the apex of sepals are straight in Amorimia subg. Amorimia, and revolute in Amorimia subg. Uncinae (figure 10).

Elaiophores - The elaiophores (sepal glands) in Amorimia follow an interesting color pattern that easily differentiates both subgenera. Those from Amorimia subg. Amorimia vary in color from green, yellow, orange to red at age (figure 11). While the elaiophores of Amorimia subg. Uncinae vary in color from green to ocher at age (figure 11). Naturalists and modern systematists have long ignored shape and color of elaiophores in Malpighiaceae in their classification systems. Future broad taxonomic studies in Malpighiaceae should pay attention to this neglected character, which might reveal itself as important as it is in Amorimia. 


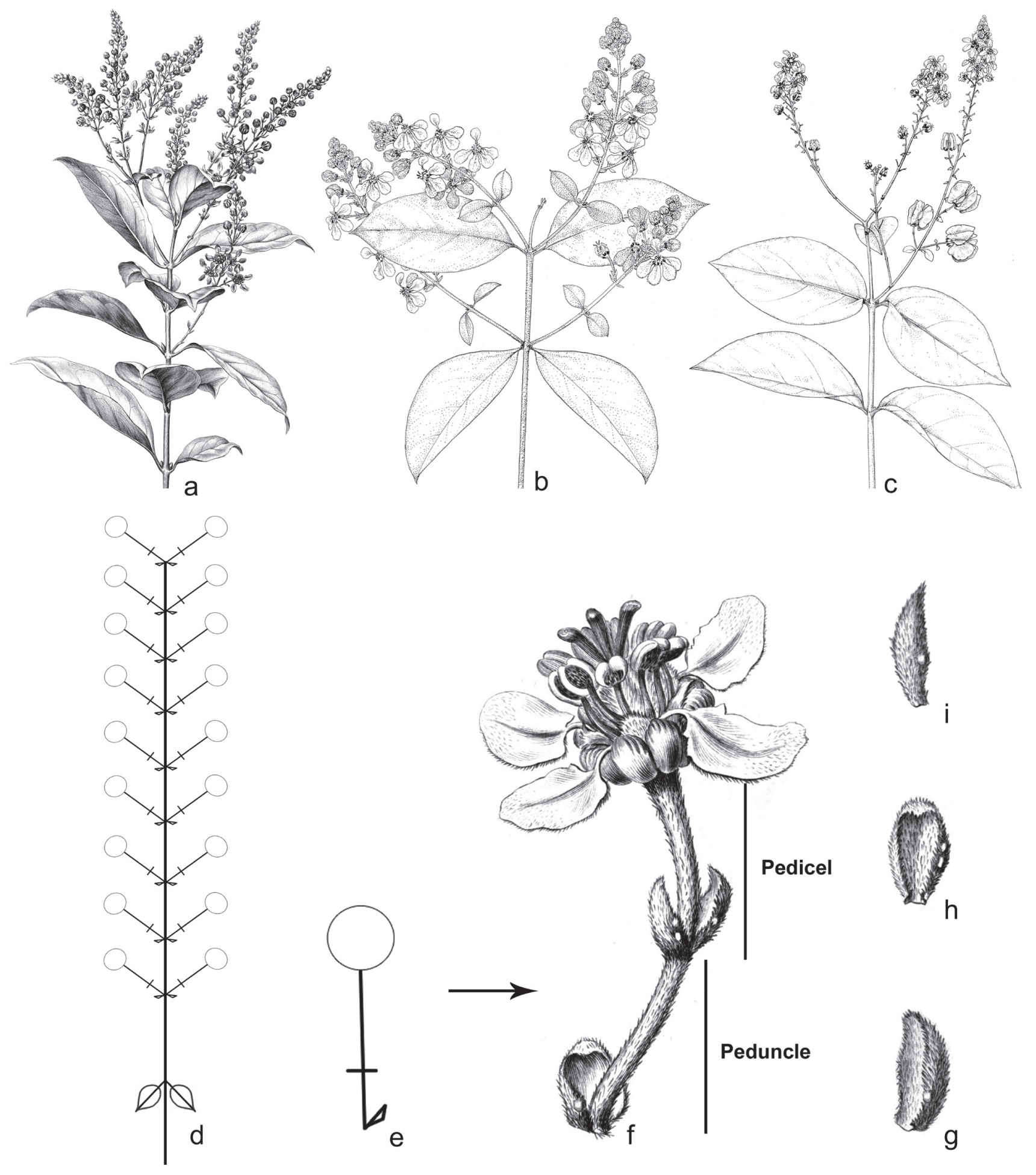

Figure 9. Inflorescence architecture in Amorimia. a. panicles of Amorimia coriacea. b. thyrsi of Amorimia kariniana. c. panicle and thyrsi of Amorimia rigida. d. thyrse bearing a pair of reduced leaves at base, and opposite to spirally alternate 1-flowered cincinni. e. 1-flowered cincinnus. f. 1-flowered cincinnus showing the peduncle bearing a bract at base and a pair of bracteoles at apex, and the pedicel bearing the flower. g. side view of a bract with a gland. h. bracteole in adaxial view evidencing two glands. i. bracteole in side view showing a gland (drawings a and f-i by Grisebach, b-c by Karin Weishaar Douthit, d-e by R.F. Almeida). 

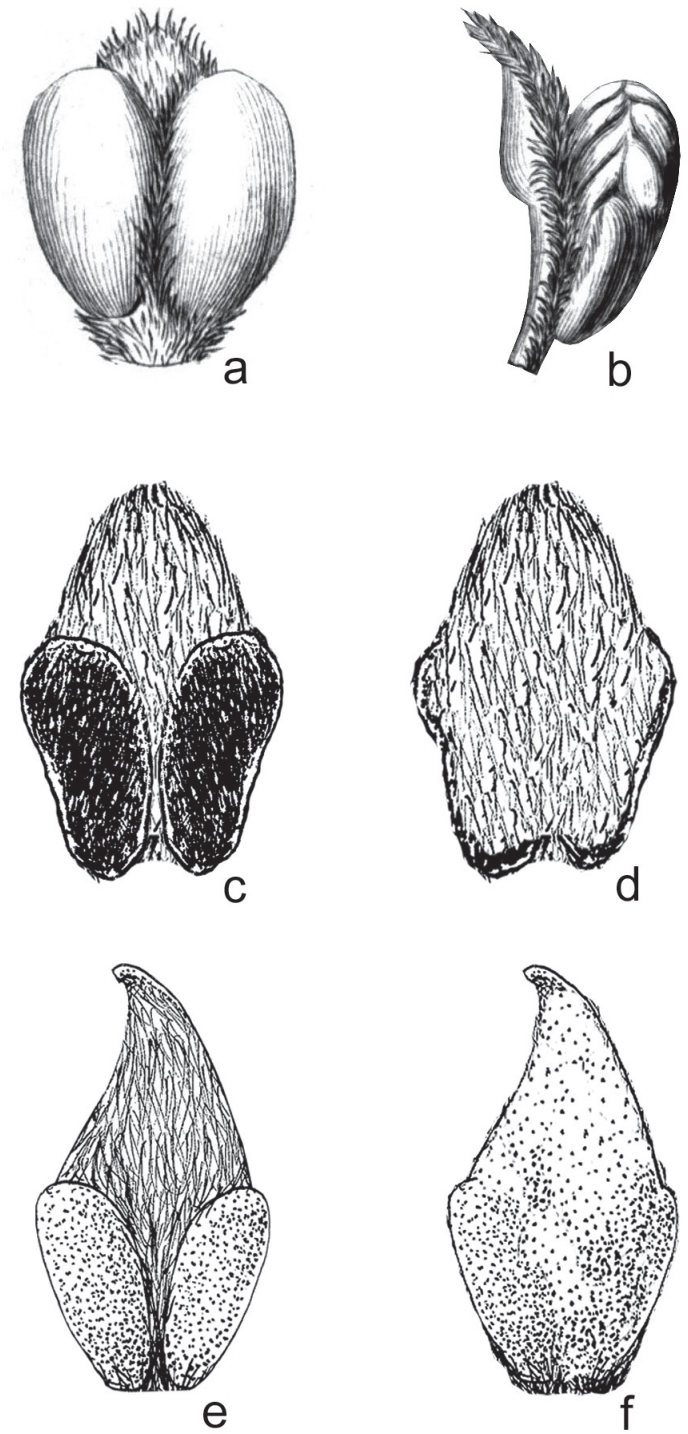

Figure 10. Indumenta and shape of sepals in Amorimia. a. sepal in abaxial view. b. sepal in side view showing an elaiophore. c. sepal in abaxial view showing rounded apex. d. sepal sericeous-velutine in adaxial view. e. sepal in abaxial view showing acute apex. $f$. sepal glabrous in adaxial view (drawings a-b by Grisebach, c-f by Karin Weishaar Douthit).

Petals - The petals in Amorimia are mostly elliptic, but they might also vary from wide-elliptic to obovate (figures 12-13). The margins are always entire, the base of the petal limbs might vary from truncate to cuneate, and the claws might vary in length and from canaliculate to plane (figures 12-13). The abaxial side is consistently pubescent in all species, but only adaxially pubescent in species from the Amorimia subg. Amorimia (figures 12-13). All species show plane petals at anthesis, except A. concinna, which shows cucullate lateral-petals at anthesis (figures 12-13).
Androecium - The androecium in Amorimia is composed of two whorls of five stamens each, both connate at base in a single ring of stamens, as most genera of Malpighiaceae (Anderson 1981). The filaments are usually glabrous in most species, being entirely pubescent or pubescent only at apex in $A$. tumida and $A$. andersonii, respectively. The length of filaments in species from Amorimia subg. Amorimia might vary from filaments opposite petals shorter than those opposite sepals (A. andersonii, A. coriacea, and A. exotropica); to filaments opposite petals longer than those opposite sepals (A. candidae, A. maritima, $A$. pellegrinii, and $A$. velutina); or filaments opposite the anterior sepal, lateral-posterior sepals and posterior petal longer than those opposite the lateral petals ( $A$. rigida) (figure 14). In species from Amorimia subg. Uncinae, the length of stamens filaments might vary from filaments opposite petals longer than those opposite sepals (A. amazonica, A. camporum, A. pubiflora, and $A$. septentrionalis) to filaments opposite petals shorter than those opposite sepals (A. concinna, and A. kariniana) (figure 14).

The anthers in Amorimia are mostly elliptic, erect at anthesis and reflexed at post anthesis (figure 14). The connectives are covered by a glandular tissue, easily visualized under a stereomicroscope (figure 15). However, it does not form a protuberant gland as in other genera of Malpighiaceae (e.g., Banisteriopsis C.R.Rob. and Stigmaphyllon A.Juss.) (Anderson 1997, Gates 1982). The pollen sacs are rimose and commonly liberate pollen grains during the pre-anthesis, inside the floral buds. The indument of anthers shows significant taxonomic relevance in distinguishing species of Amorimia (figure 15). The anthers might be entirely pubescent (i.e., A. candidae, $A$. pelegrinii, and $A$. rigida), pubescent only at apex and/or base (i.e., A. andersonii, A. pubiflora and $A$. septentrionalis), pubescent at connectives and base (i.e., A. coriacea, A. exotropica, A. maritima, and A. velutina), or entirely glabrous (i.e., A. amazonica, $A$. camporum, A. concinna, and A. kariniana) (figure 15).

Gynoecium - All species of Amorimia show densely sericeous-velutine to velutine ovaries, bearing primordial dorsal and lateral wings (figure 16). The styles are usually cylindrical at base and flattened towards the apex, entirely glabrous, and pubescent at base and middle (pubescent at apex only in A. tumida) (figure 16). The shape of the apex of styles is an important character that distinguishes both subgenera in Amorimia. styles truncate at apex are diagnostic of 

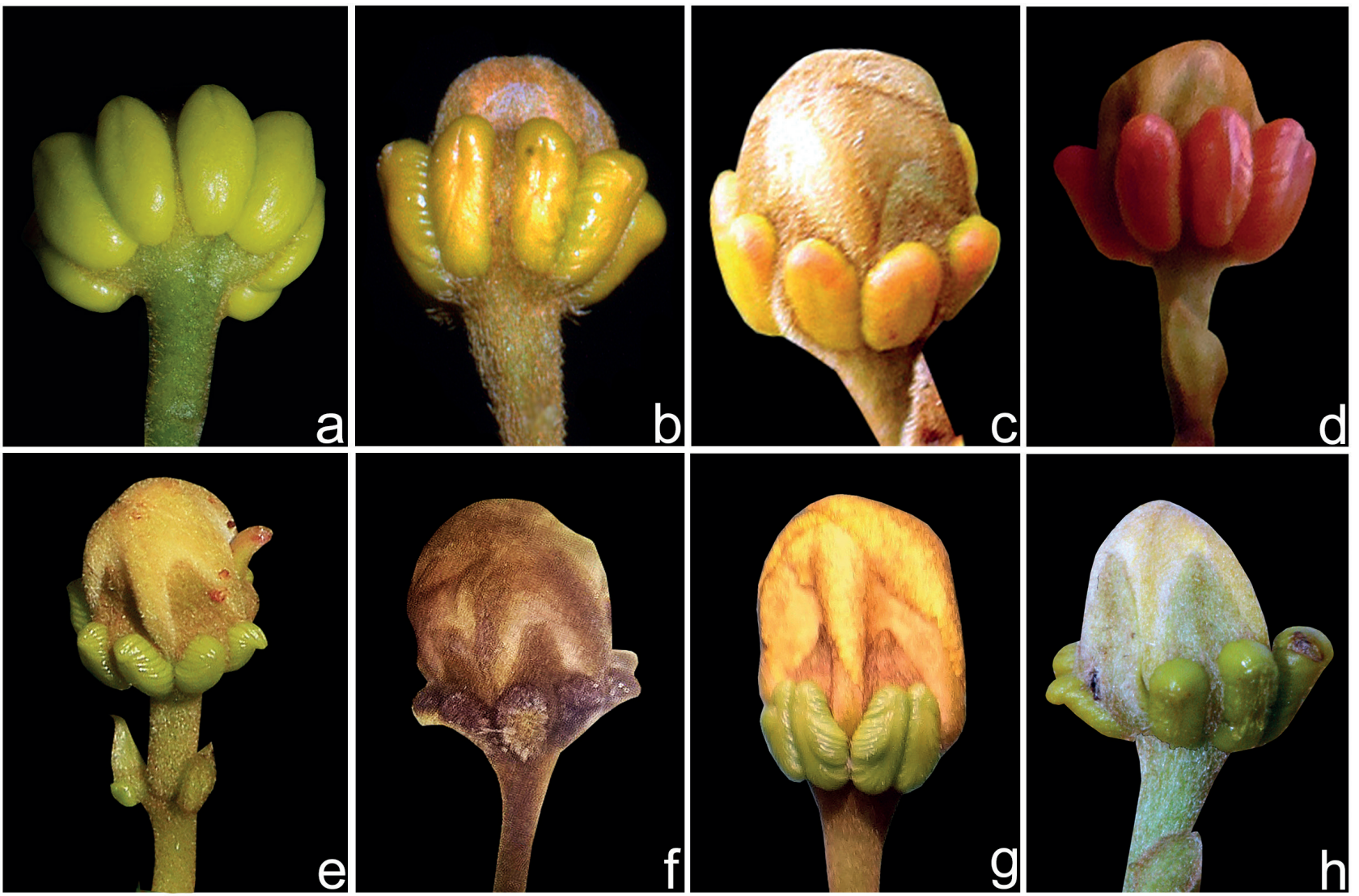

Figure 11. Color of elaiophores in Amorimia subg. Amorimia. a. green - Amorimia andersonii. b. yellow - Amorimia coriacea. c. orange - Amorimia candidae. d. red - Amorimia maritima. Color of elaiophores in Amorimia subg. Uncinae: e. green - Amorimia amazonica. f. ocher - Amorimia camporum. g. green - Amorimia pubiflora. h. green - Amorimia septentrionalis (photographs a by F. Michelangeli, b, h by M.O.O. Pellegrini, d by F. Flores, e by H. Medeiros, $f$ by R.F. Almeida, g by E. Moletta).

species belonging to $A$. subg. Amorimia, while Styles uncinate at apex are diagnostic of species belonging to A. subg. Uncinae (figure 16).

Fruits - The fruit in Amorimia is a schizocarp, separating into three winged mericarps (Anderson 2006). All mericarps resemble a bow tie due to two lateral wings longer than the dorsal wing (figure 17). The dorsal wing might be consistently rounded (all species of $A$. subg. Uncinae, and $A$. candidae from A. subg. Amorimia) to triangular, sericeous-velutine to velutine, and sometimes bears a projection at apex (e.g., A. coriacea, and A. velutina) (figure 17). The lateral wings are usually flabellate, sinuate, sericeousvelutine to velutine, and might vary on the angle of insertion in the upper or lower part of the nut (figure 17). The nuts are always ovoid, smooth, and densely sericeous-velutine to velutine (figure 17). The areole is the insertion of the mericarp into the floral receptacle, and might vary in shape from narrow to wide-elliptic (figure 17).
Seed and Embryo - The seed in Amorimia is usually ovoid with a smooth testa, uniquely rugose in $A$. tumida (figure 18). The embryos are consistently ovoid, showing folded cotyledons (figure 18). I noticed that most species show well-developed seeds bearing an embryo and cotyledons with still moist and likely viable tissue, even in seeds from herbarium specimens from the 1970's (i.e., A. amazonica). It is possible that these seeds are still viable, making it possible to perform a study on chromosome numbers in the genus. Amorimia is the only Neotropical genus of the Malpighioid clade with still unknown chromosome numbers (pers. obs.).

\section{Taxonomy}

Amorimia W.R.Anderson, Novon 16(2): 176. 2006. Type: Amorimia rigida (A.Juss.) W.R.Anderson.

= Mascagnia sect. Pleuropterys Griseb., Fl. Bras. 12(1): 91. 1858. Lectotype (designated by Anderson 

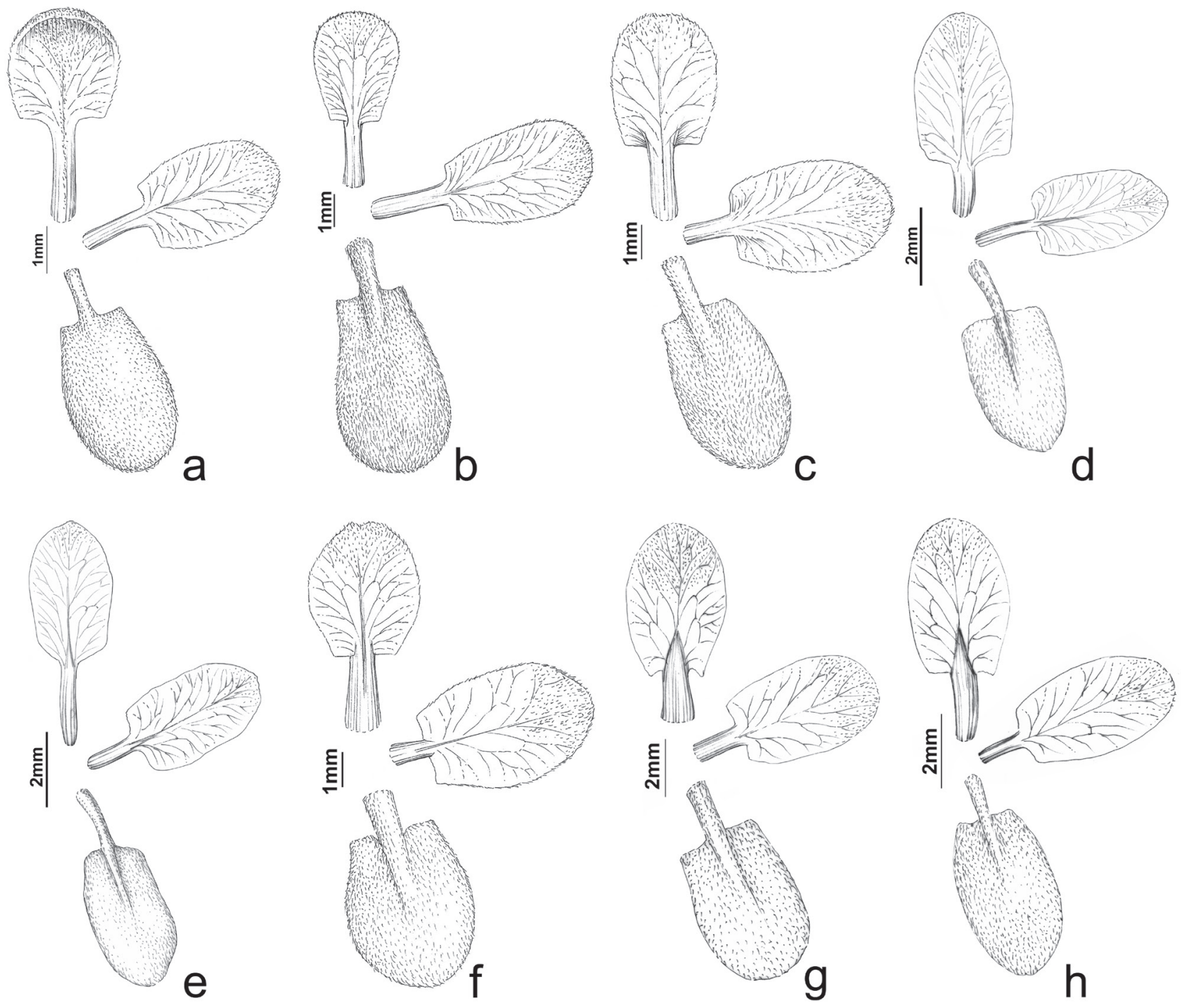

Figure 12. Shape of petals from Amorimia subgenus Amorimia (posterior petal/posterio-lateral petal/anterio-lateral petal). a. Amorimia andersonii. b. Amorimia candidae. c. Amorimia coriacea. d. Amorimia exotropica. e. Amorimia maritima. f. Amorimia pellegrinii. g. Amorimia rigida. h. Amorimia velutina (drawings by Klei Sousa).

2006): Hiraea pubiflora A. Jussieu, Fl. Bras. Mer. (quarto ed.) 3(21): 14. $1833 \equiv$ Mascagnia pubiflora (A.Juss.) Griseb.Fl.Bras. 12(1):91.1858 $\equiv$ Amorimia pubiflora (A.Juss.) W.R.Anderson, Novon 16(2): 183. 2006.

Figures 2-40

Woody vines to scandent shrubs; indumenta from throughout the plant varying from sericeous-velutine (composed of a mixture of short $\mathrm{T}$ and V-Y-shaped unicellular hairs) to velutine (composed only by V-Yshaped hairs); stipules minute, triangular, interpetiolar in vegetative branches, epipetiolar in inflorescence branches, persistent to deciduous. Leaves usually opposite, sometimes subopposite to alternate near inflorescences, often reduced in inflorescences; petioles canaliculate, eglandular, rarely biglandular at apex; leaf blade narrowly elliptic to elliptic to widely elliptic, base obtuse or cuneate to attenuate, apex acute to acuminate to mucronate, eglandular or bearing 2-many small glands impressed abaxially, near midvein, base or margins. Thyrsi (pseudoracemes) to panicles, axillary to terminal; cincinni 1-flowered, decussate to spirally arranged; bracts parallel, spreading to reflexed, elliptic, ovate to lanceolate, bearing 1-2(-3) pairs of glands; peduncles short to elongate; bracteoles borne at or below apex of peduncles, parallel, spreading to reflexed, elliptic, ovate, lanceolate to filiform, eglandular to 1(-2) pairs of glands. Flowers bisexual, zygomorphic, chasmogamous; pedicels short to elongate, sometimes thickened in fruits; 

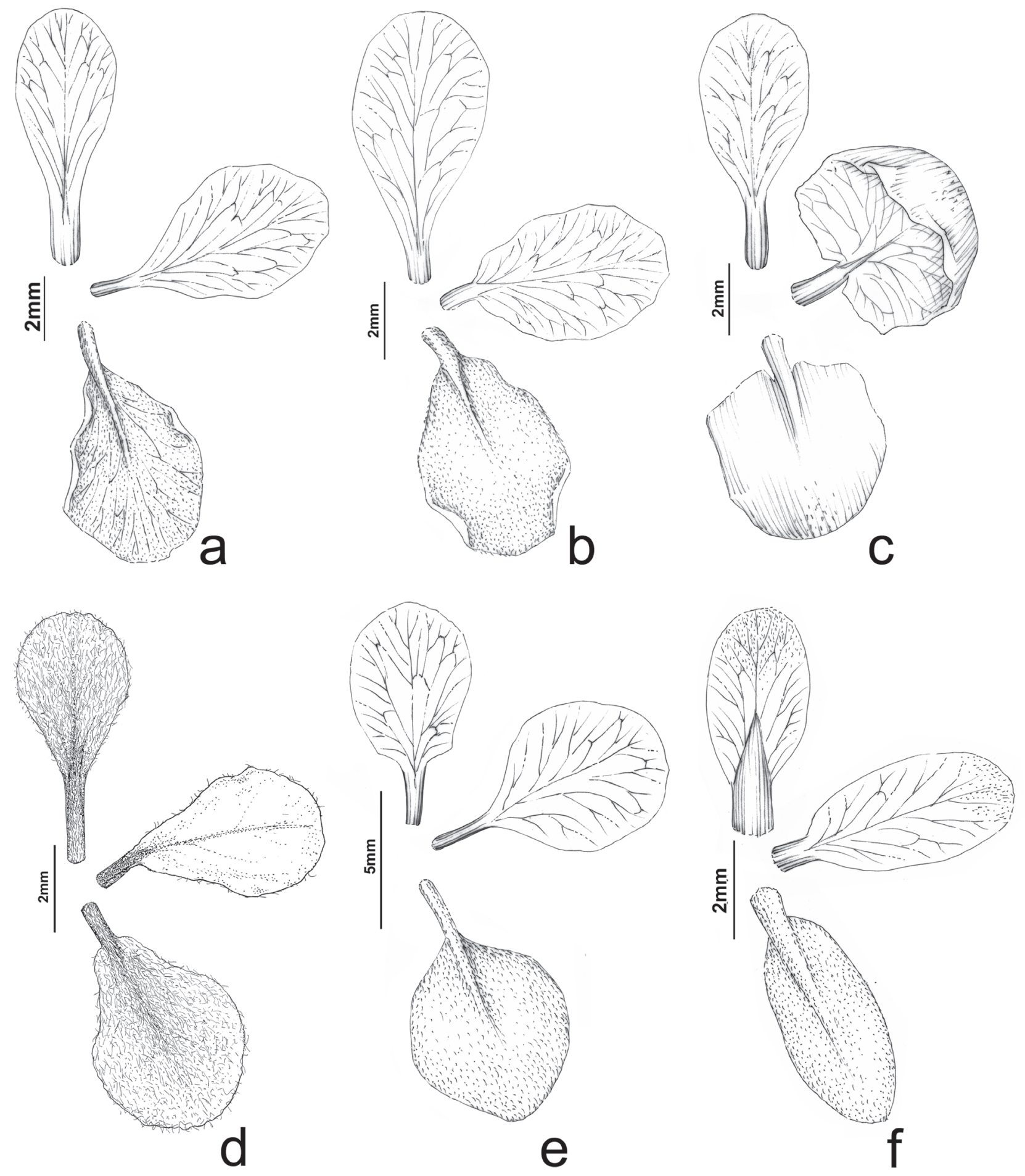

Figure 13. Shape of petals from Amorimia subgenus Uncinae (posterior petal/posterio-lateral petal/anterio-lateral petal). a. Amorimia amazonica. b. Amorimia camporum. c. Amorimia concinna. d. Amorimia kariniana. e. Amorimia pubiflora. f. Amorimia septentrionalis (drawings a-c, e-f by Klei Sousa, d by Karin Weishaar Douthit). 

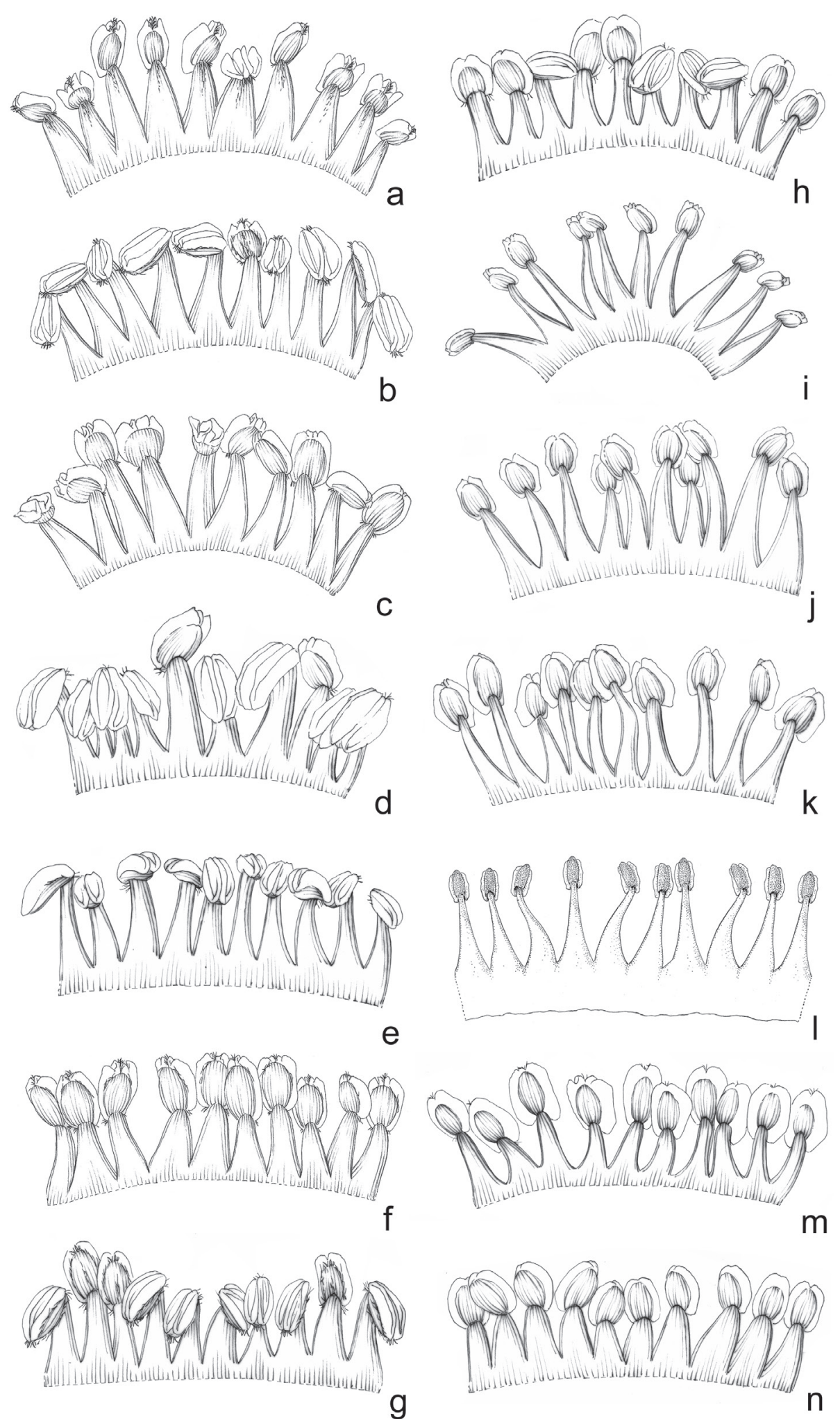

Figure 14. Androecium morphology (the first stamen to the left is always the stamen opposite the anterior sepal) in Amorimia subg. Amorimia. a. Amorimia andersonii. b. Amorimia candidae. c. Amorimia coriacea. d. Amorimia exotropica. e. Amorimia maritima. f. Amorimia pellegrinii. g. Amorimia rigida. h. Amorimia velutina. Androecium morphology in Amorimia subg. Uncinae: i. Amorimia amazonica. j. Amorimia camporum. k. Amorimia concinna. 1. Amorimia kariniana. m. Amorimia pubiflora. n. Amorimia septentrionalis (drawings a-k, m-n by Klei Sousa, 1 by Karin Weishaar Douthit). 

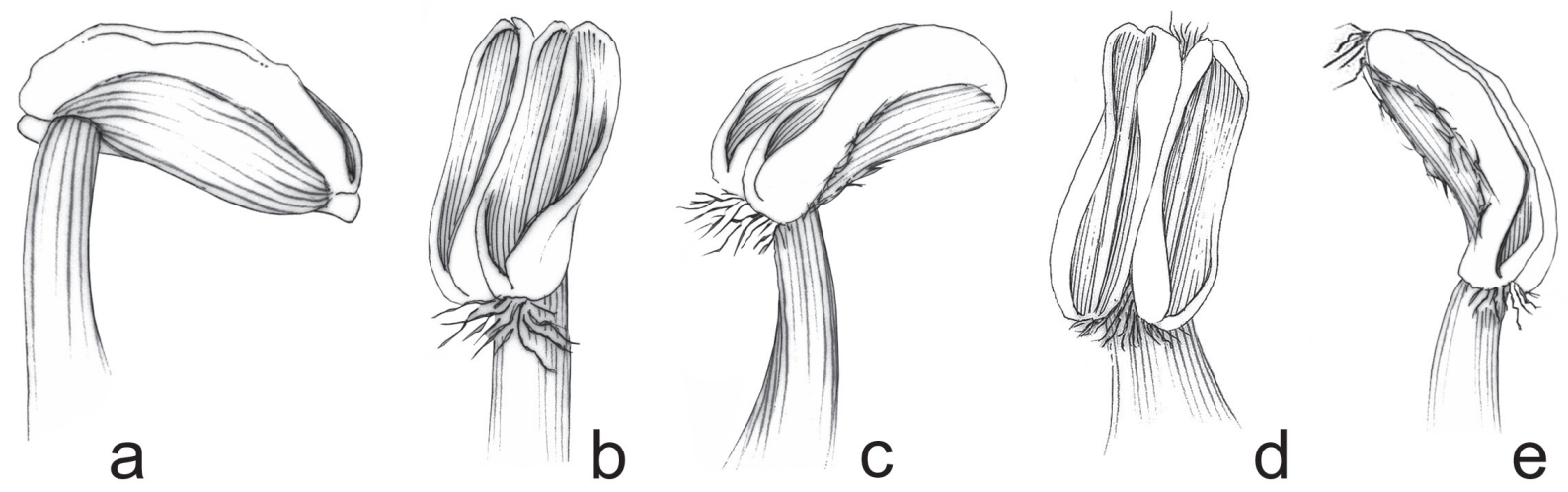

Figure 15. Indumentum arrangement in anthers. a. glabrous. b. pubescent at base. c. pubescent at base and apex. d. pubescent at base and apex. e. pubescent at base, connective and apex (drawings by Klei Sousa).

sepals leaving petals exposed during enlargement of bud, apex appressed to the androecium or revolute at anthesis; lateral sepals bearing 2 glands abaxially; the anterior eglandular, rarely bearing 1 gland; petals clawed, yellow, usually turning orange or red at age, adaxially sericeous-velutine to velutine distally or sometimes uniformly, abaxially uniformly sericeousvelutine to velutine; limb elliptic to wide elliptic to spatulate to obovate to orbicular, base cuneate to hastate to truncate to obtuse, margin entire, sinuate to plane, apex rounded, claw plane to canaliculate, posterior petal erect, lateral petals usually reflexed in age. Stamens 10, all fertile, filaments connate at base, straight, varying from longer opposite sepals to longer opposite petals, anthers monomorphic, rarely dimorphic, glabrous or pubescent at base, connective and/or apex. Gynoecium with carpels connate their whole length in the ovary, styles cylindrical or laterally flattened toward apex, erect to recurved, subequal or the anterior somewhat shorter, slenderer, and more recurved than the posterior 2, apex of styles dorsally rounded or truncate or acute to short-hooked, stigma lateral, crateriform to discoid. Fruits dry, splitting into 3 samaroid mericarps, detaching from a short or moderately high pyramidal torus; samara butterflyshaped to depressed-elliptical with lateral wings
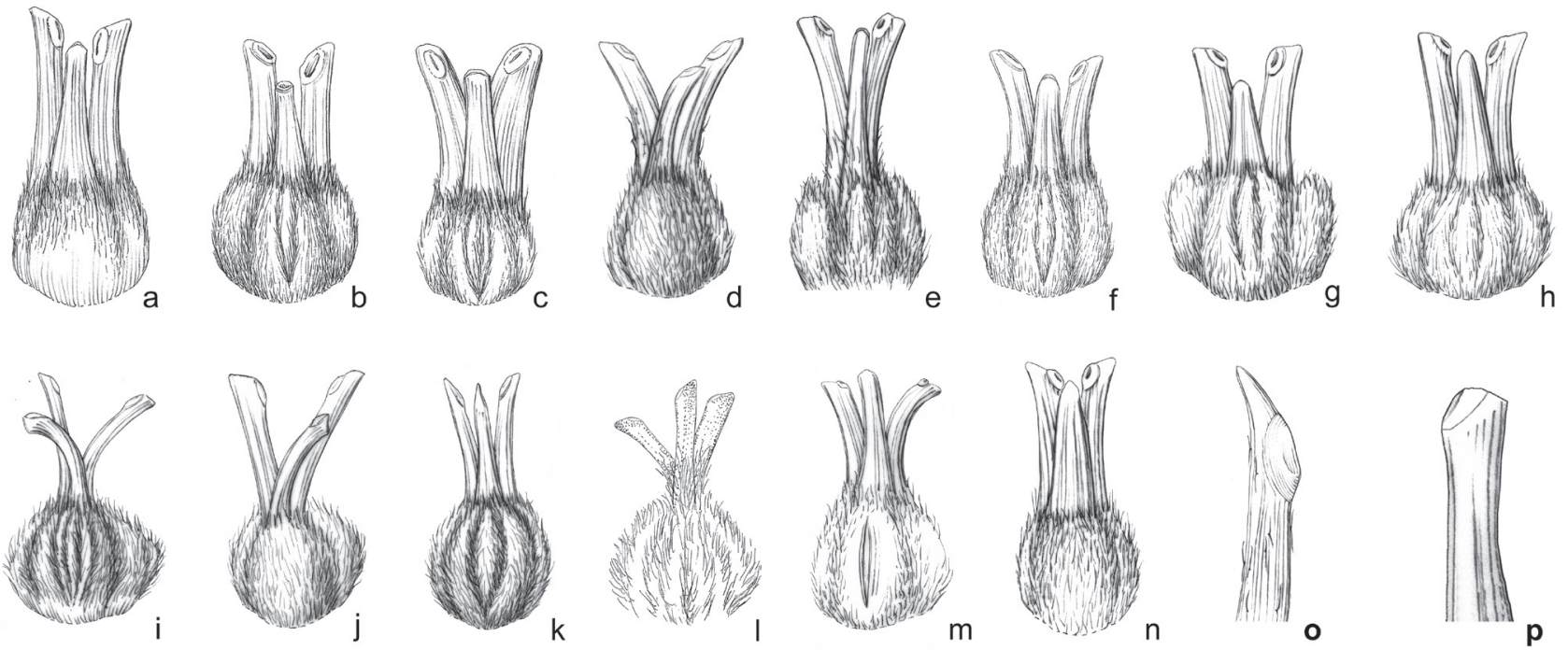

Figure 16. Gynoecium morphology of Amorimia subg. Amorimia. a. Amorimia andersonii. b. Amorimia candidae. c. Amorimia coriacea. d. Amorimia exotropica. e. Amorimia maritima. f. Amorimia pellegrinii. g. Amorimia rigida. h. Amorimia velutina. Gynoecium morphology of Amorimia subg. Uncinae: i. Amorimia amazonica. j. Amorimia camporum. k. Amorimia concinna. 1. Amorimia kariniana. m. Amorimia pubiflora. n. Amorimia septentrionalis. o. style with uncinate apex. p. style with truncate apex (drawings by Klei Sousa). 


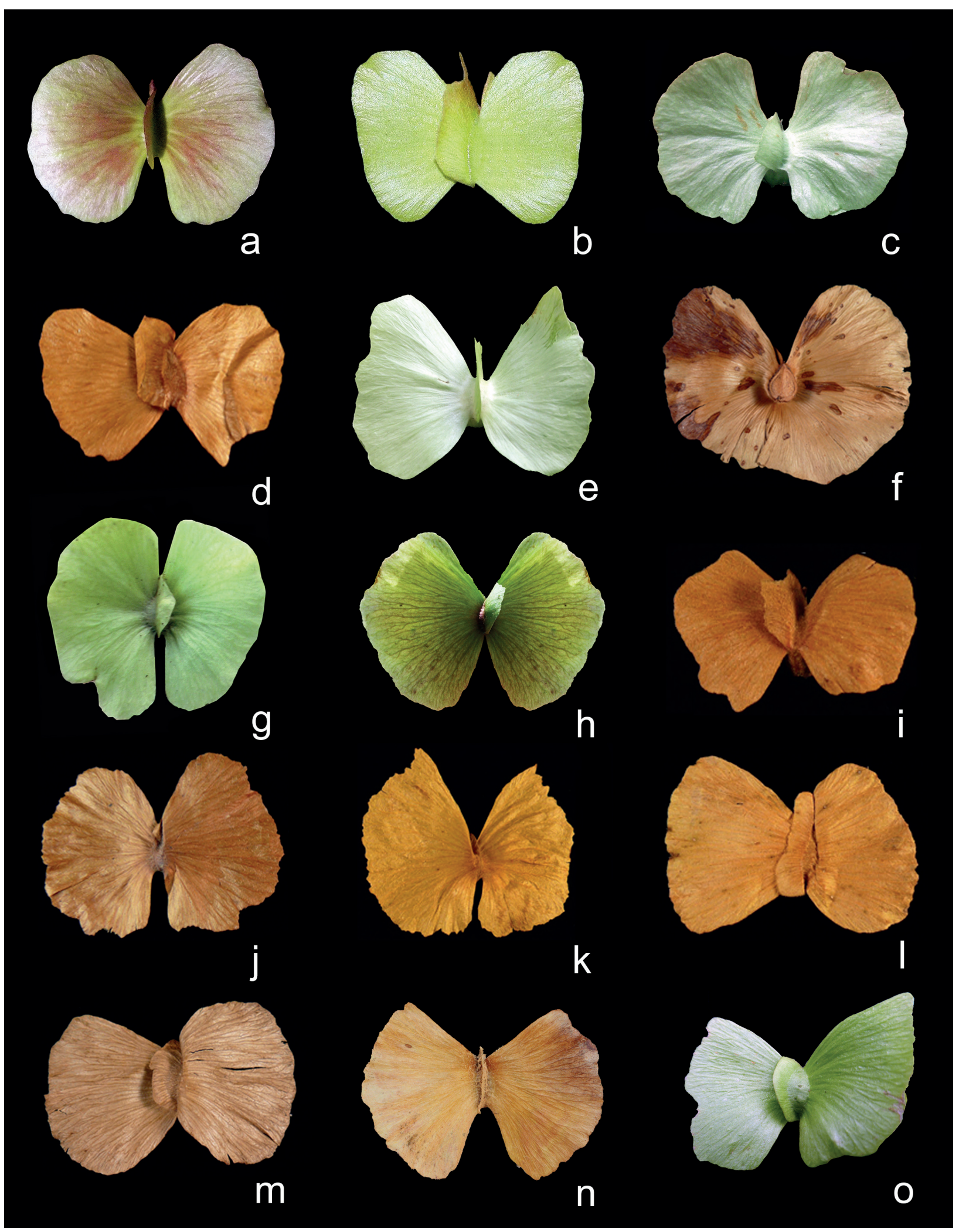

Figure 17. Samara morphology of Amorimia subg. Amorimia. a. Amorimia candidae. b. Amorimia coriacea. c. Amorimia exotropica. d. Amorimia exotropica. e. Amorimia maritima. f. Amorimia maritima. g. Amorimia pellegrinii. h. Amorimia rigida. i. Amorimia velutina. Samara morphology of Amorimia subg. Uncinae: j. Amorimia amazonica. k. Amorimia camporum. 1. Amorimia concinna. m. Amorimia pubiflora. n. Amorimia septentrionalis. o. Amorimia tumida (photographs a, c, e, g, h, i by R.F. Almeida, d, f, j, k, l, m by W.R. Anderson, b by C.N. Fraga, o by M.N. Coelho). 


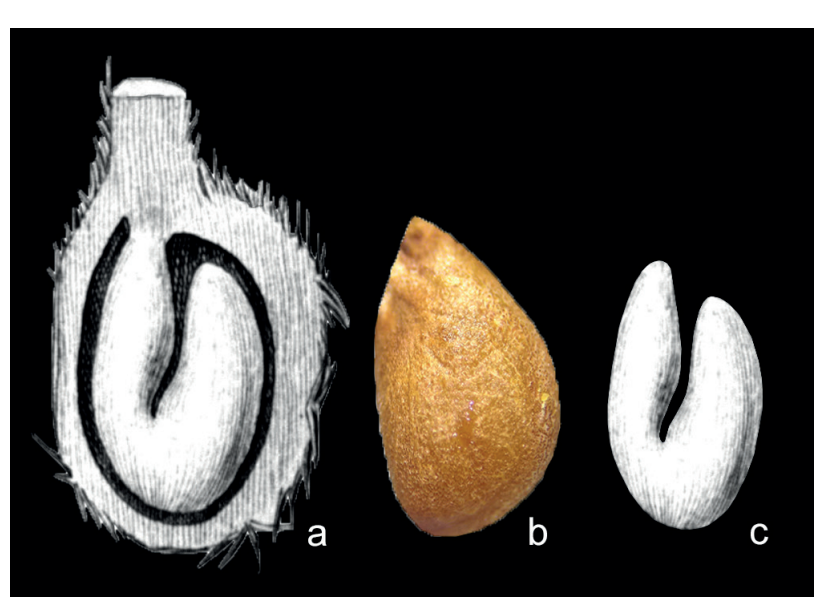

Figure 18. Seed and embryo morphology in Amorimia. a. nut in side view showing an embryo with cotyledons folded. b. seed in side view showing a smooth testa. c. embryo showing its cotyledons folded (a,c by A. Grisebach, b by R.F. Almeida).

dominant, chartaceous with many fine parallel veins, cleft towards nut at apex, continuous at base or cleft part-way or completely towards nut, margin entire or sinuate to coarsely dentate; dorsal wing distinct at apex and base or confluent with lateral wings at base; nut almost always smooth between lateral and dorsal wings; ventral areole triangular, ovate, orbicular to very narrowly elliptical. Seeds smooth to rugose; embryos narrowly ovoid to ovoid, cotyledons bent.

Etymology - The genus honors the Brazilian botanist André Márcio Araujo Amorim (b. 1966), contributor to the study of the Neotropical genus Heteropterys (Malpighiaceae).

Distribution and ecology - Amorimia comprises 15 species found in Evergreen and Seasonally Dry Tropical Forests, usually associated to annual or perennial water streams in South America (figures 2, 4).
Recommendations for field collectors - Most Amorimia species are differentiated by the morphology of their indumenta, bracts/bracteoles, flowers and fruits. Therefore, it is recommended, whenever possible, to collect young vegetative branches, as well as reproductive branches bearing flowers and fruits, besides recording the color of glands, petals and fruits during pre and post-anthesis.

\section{Key to the subgenera of Amorimia}

1. Sepals abaxially pubescent, petals elliptic, base truncate to hastate, claws canaliculate, styles truncate at apex, acuminate to rounded (slightly uncinate in $A$. andersonii), pollen grain polygonal . Amorimia subg. Amorimia

1. Sepals abaxially glabrous, petals obovate to spatulate, base cuneate, claws plane, styles uncinate at apex, pollen grain spherical . Amorimia subg. Uncinae

1. Amorimia W.R.Anderson subg. Amorimia. Type: Amorimia rigida W.R.Anderson.

Differs from A. subg. Uncinae R.F. Almeida by its stipules triangular; bracteoles concave; sepals adaxially pubescent; petals elliptic, base truncate to hastate, adaxially entirely to distally pubescent, claws canaliculate; anthers always pubescent (at base, connective and/or apex); styles with apex truncate, acuminate to rounded; pollen grains polygonal; samaras with dorsal wing usually confluent with lateral wings at base.

Notes - This subgenus includes eight species (i.e., $A$. andersonii, A. candidae, A. coriacea, A. exotropica, A. maritima, A. pellegrinii, A. rigida, and A. velutina) mostly restricted to SDTF to Rainforests from Eastern South America (figure 19).

\section{Key to the species of Amorimia subg. Amorimia}

1. Leaves lanceolate elliptic to narrowly elliptic, sparsely velutine to glabrous at age, with 8-16 pairs of secondary veins; bracts and bracteoles deflexed, bracteoles inserted below apex of peduncle; sepals erect with revolute apex at anthesis, glands turning ocher, filaments pubescent, styles uncinate at apex; samaras with dorsal wing depressed ovate; Atlantic Forest Inselbergs from southern State of Bahia

1. Leaves ovate to elliptic to widely elliptic to orbicular, sericeous-velutine to glabrous at age, with 8 or fewer pairs of secondary veins; bracts and bracteoles parallel, bracteoles inserted at apex of peduncle; sepals appressed to the androecium with straight apex at anthesis, glands turning brown or red, petals adaxially proximally glabrous, filaments glabrous, styles truncate at apex; samaras with dorsal wing obtrapezoidal or triangular; Caatinga, Cerrado and Atlantic Forest domains 
2. Claw of the posterior petal up to $1 \mathrm{~mm}$ long; stamens opposite the anterior sepal, posteriorlateral sepals and posterior petal longer than those opposite lateral petals; Atlantic Forest from States of Bahia and Minas Gerais, Brazil A. rigida

2. Claw of the posterior petal more than $1 \mathrm{~mm}$ long; all stamens opposite petals longer or shorter than those opposite sepals

3. Stamens opposite petals shorter than those opposite sepals

4. Leaves conduplicate; peduncles exceeding bracts at anthesis, peduncles equaling pedicels at anthesis; restinga from State of Rio de Janeiro, Brazil

A. coriacea

4. Leaves plane; peduncles not exceeding bracts at anthesis, peduncles shorter than pedicels at anthesis; SDTFs and Rainforests from Southern Brazil, Argentina and Paraguay

3. Stamens opposite petals longer than those opposite sepals

5. Petals turning only red at post-anthesis, posterior petal 2-glandular at base of limb; samaras with dorsal wing trapezoidal with rounded angles; Caatinga from central Bahia State, Brazil ... A. candidae

5. Petals turning orange to red at post-anthesis, posterior petal eglandular; samaras with dorsal wing triangular or obtrapezoidal bearing a projection at apex

6. Lateral petals patent at anthesis, anthers pubescent at apex, connectives and base; samaras with dorsal wing triangular; Caatinga from Northeastern Brazil ..... A. pellegrinii

6. Lateral petals reflexed at anthesis, anthers pubescent only at base and/or connectives; samaras with dorsal wing obtrapezoidal

7. Leaves bullate; peduncles as long as pedicels at anthesis; anterior-lateral petals overlapping; anthers pubescent only at base; samaras with dorsal wing bearing two aciculate projections at apex; SDTF from States of Bahia, Goiás, and Minas Gerais, Brazil A. velutina

7. Leaves not bullate; peduncles shorter than pedicels at anthesis; anterior-lateral petals divergent; anthers pubescent at base and connectives; samaras with dorsal wing bearing a reduced single aciculate projection at apex; Atlantic Forest from States of Bahia, Espírito Santo and Rio de Janeiro, Brazil

A. maritima

1.1. Amorimia andersonii R.F.Almeida, Phytotaxa 284(1): 4. 2016. Holotype: BRAZIL. Bahia: Mun. Itamarajú, Serra ao lado do Morro do Pescoço, entrando pela Fazenda Novo Horizonte e passando pela propriedade do Sr. Ailton, área rochosa no alto da serra, 16-II-2014, fl., L.C. Marinho, A.M.A. Amorim, R. Goldenberg \& L. Daneu 654 (CEPEC!; isotypes: HUEFS!, P!, RB!).

Figures 20, 22

Woody vines to scandent shrubs; branches smooth, sparsely lenticellate, lenticels inconspicuous, sparsely velutine to glabrous at age; stipules 0.4-0.6 mm long, triangular, glabrous, interpetiolar on branches, epipetiolar on inflorescences, deciduous. Leaves subopposite, not reduced in inflorescences; petioles 6.5-8 $\mathrm{mm}$ long, canaliculate, sparsely sericeousvelutine to glabrous at age, eglandular at apex; leaf blades 5.15-11.5 × 1.6-4.3 cm, plane, not bullate, lanceolate to elliptic to narrow-elliptic, base cuneate to attenuate, margin revolute, apex acute, adaxially glabrous, abaxially sparsely velutine to glabrous, 1 pair of glands, $0.6-0.7 \mathrm{~mm}$ diam., near base or up to 0.8-0.9 mm of margins; midvein adaxially impress, abaxially prominent, $10-16$ pairs of secondary veins, arching $60-64^{\circ}$, subopposite to alternate, adaxially impress, abaxially prominent, reticulum adaxially impressed and inconspicuous, abaxially prominent and conspicuous. Thyrsi (pseudoracemes), axillary; main axis 9.4-15.2 cm long, cylindrical, smooth, velutine; cincinni 20-40, 1-flowered, proximally spirally alternate, distally decussate; reduced leaves absent; bracts 3.5-5 $\times 1-1.2 \mathrm{~mm}$, lanceolate, plane, subsessile, deflexed to the peduncle, 1 pair of marginal glands near base, both sides minutely velutine; peduncle $5-8 \times 1-1.3 \mathrm{~mm}$, cylindrical, velutine; bracteoles 1.5-2.5 $\times 1.0-1.2 \mathrm{~mm}$, elliptic, concave, sessile, inserted $0.5-2 \mathrm{~mm}$ below the apex of peduncles, opposite to subopposite, reflexed to the peduncle, 1-2 glands near base, both sides velutine. Flowers 13-14 mm diam. at anthesis; floral buds 5-6 $\times 4-5 \mathrm{~mm}$ at anthesis; pedicels 6-7.5 $\times 1.5-1.7 \mathrm{~mm}$, cylindrical, velutine. Sepals 0.5-1 × 0.5-0.55 mm, narrowly oblong, not appressed to the androecium, apex acute, 
obtuse to rounded, revolute at anthesis, both sides sericeous-velutine; glands yellow turning ocher at age, $1-1.2 \times 0.3-0.5 \mathrm{~mm}$. Petals bright yellow, not turning darker at age, margin sinuate, anterior-lateral petals overlapping; lateral petals patent at anthesis, limb 2.4-3.4 × 1-1.2 mm, wide elliptic, base truncate, adaxially entirely velutine, abaxially sericeousvelutine; claws $0.5-0.8 \times 0.2-0.3 \mathrm{~mm}$, plane, adaxially velutine, abaxially sericeous-velutine; posterior petal erect at anthesis, limb 1.4-1.5 × 1.2-1.3 mm, wide elliptic, base cuneate, eglandular, adaxially velutine, abaxially sericeous-velutine; claw 1-1.2 $\times 0.3-0.4 \mathrm{~mm}$, plane, adaxially velutine, abaxially sericeousvelutine. Stamens opposite petals shorter than those opposite sepals, except for the one opposite to the anterior sepal which is shorter than others; filaments $1-1.6 \times 0.4-0.6 \mathrm{~mm}$, connate $0.4-0.5 \mathrm{~mm}$ long at base, sericeous at apex; anthers dimorphic, recurved, with a glandular connective, $0.8-1 \times 0.4-0.6 \mathrm{~mm}$, reflexed at anthesis, base, connective and apex pubescent. Ovary 1-1.1 × 0.9-1 mm, each carpel with primordial lateral and dorsal wings, sericeousvelutine; styles 3, cylindrical at base, flattened and curved at apex, parallel at base, divergent at middle, apex truncate to slightly uncinate, sericeous-velutine at base, glabrous at middle and apex, anterior style 1.2-1.4 mm long, posterior styles 1.4-1.6 mm long; stigma lateral, discoid. Samaras (immature) green in

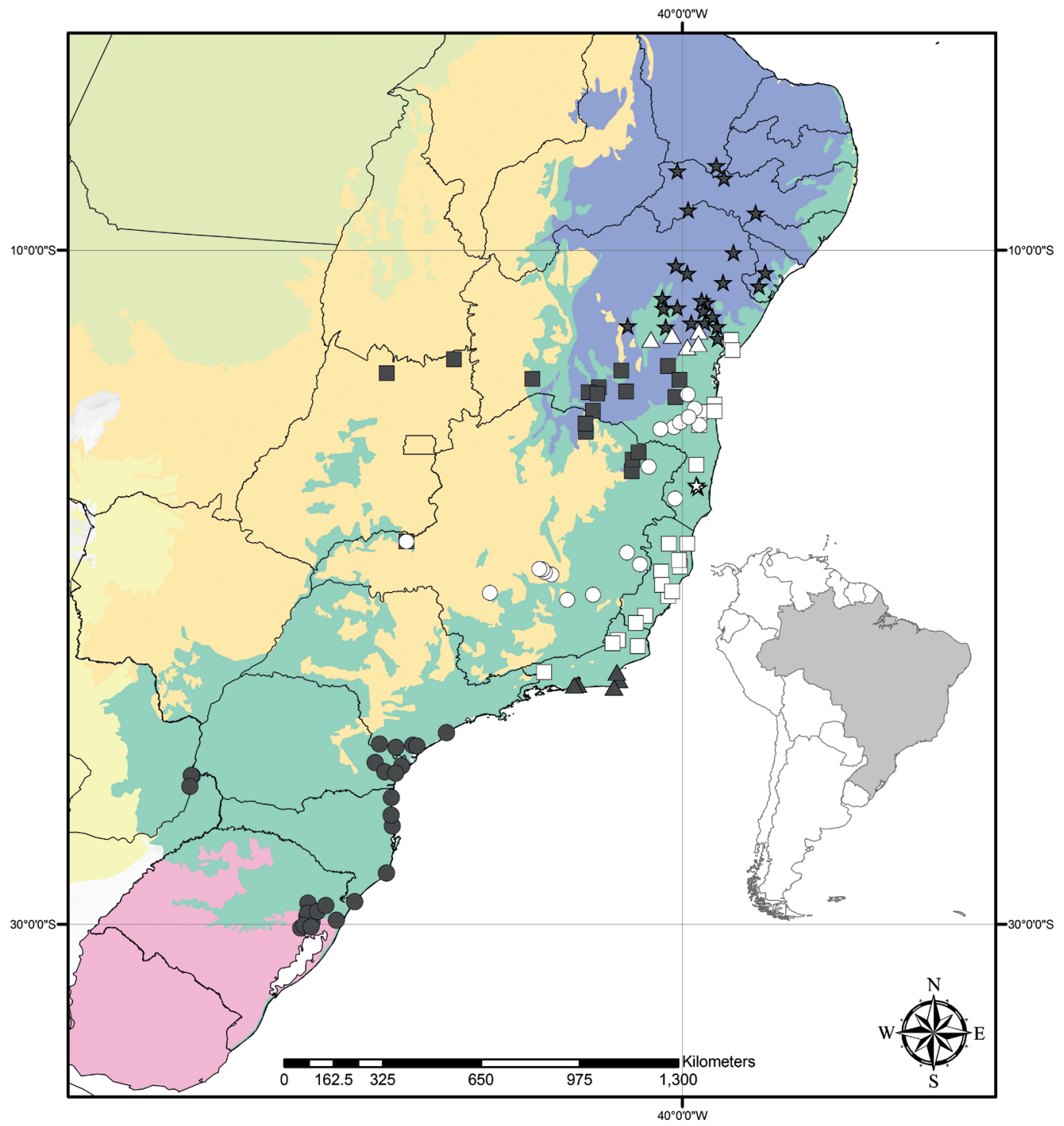

Figure 19. Distribution map of species from the Amorimia subg. Amorimia $\bullet \bullet-$ Amorimia exotropica. $\circ-$ Amorimia rigida. $\mathbf{\Delta}-$ Amorimia coriacea. $\Delta$ - Amorimia candidae. - Amorimia velutina. $\square-$ Amorimia maritima. Black star - Amorimia pellegrinii. White star - Amorimia andersonii. Pink - Pampa, dark green - Atlantic Forest, violet - Caatinga, light green - Amazon Forest, and yellow - Chaco/Pantanal. 
vivo; dorsal wing ca. $6.3 \times 1.5 \mathrm{~mm}$, depressed ovate, margin entire, plane, velutine on both sides; lateral wings 8-7.2 $\times 4-17.9 \mathrm{~mm}$, flabelliform, margin entire, sinuate, upper angle $58^{\circ}$, lower angle $63^{\circ}$ from the nut, both sides velutine to glabrous; nut ca. $1.3 \times 0.7 \mathrm{~mm}$, ellipsoid, velutine; areole ca. $1 \times 0.5 \mathrm{~mm}$. Seeds not seen.

Specimens analyzed: BRAZIL. BAHIA: Mun. Itamaraju, Morro do Pescoço, ca. $15 \mathrm{~km}$ da entrada da cidade, 11-II-2007, fl., Amorim 6856 (CEPEC); loc. cit., 16-II-2014, fl., Marinho 676 (CEPEC).

Distribution, habitat and phenology: Amorimia andersonii is only known from Semi-deciduous forests associated to rocky outcrops in Southern State of Bahia, Brazil (figure 19), flowering in February.
Conservation status: Amorimia andersonii is known from only three collections from Semi-deciduous Forests, associated to rocky outcrops within the Atlantic Forest of southern Bahia. Despite my efforts to recollect this species in December 2015, no individuals were found at Pedra do Pescoço, its type locality. Thus, this species should be regarded as Critically Endangered [CR, A3b; B1ab(v); C2a(i,ii); D1; E], due to its EOO being about $4.678 \mathrm{~km}^{2}$ and continually declining habitat quality.

Etymology: the epithet honors the North American botanist Dr. William Russell Anderson (*1942-2013†), colleague and longtime contributor in the studies of Neotropical Malpighiaceae.

Taxonomic notes: Amorimia andersonii is similar to $A$. pellegrinii and $A$. rigida, due to their plane leaf blades
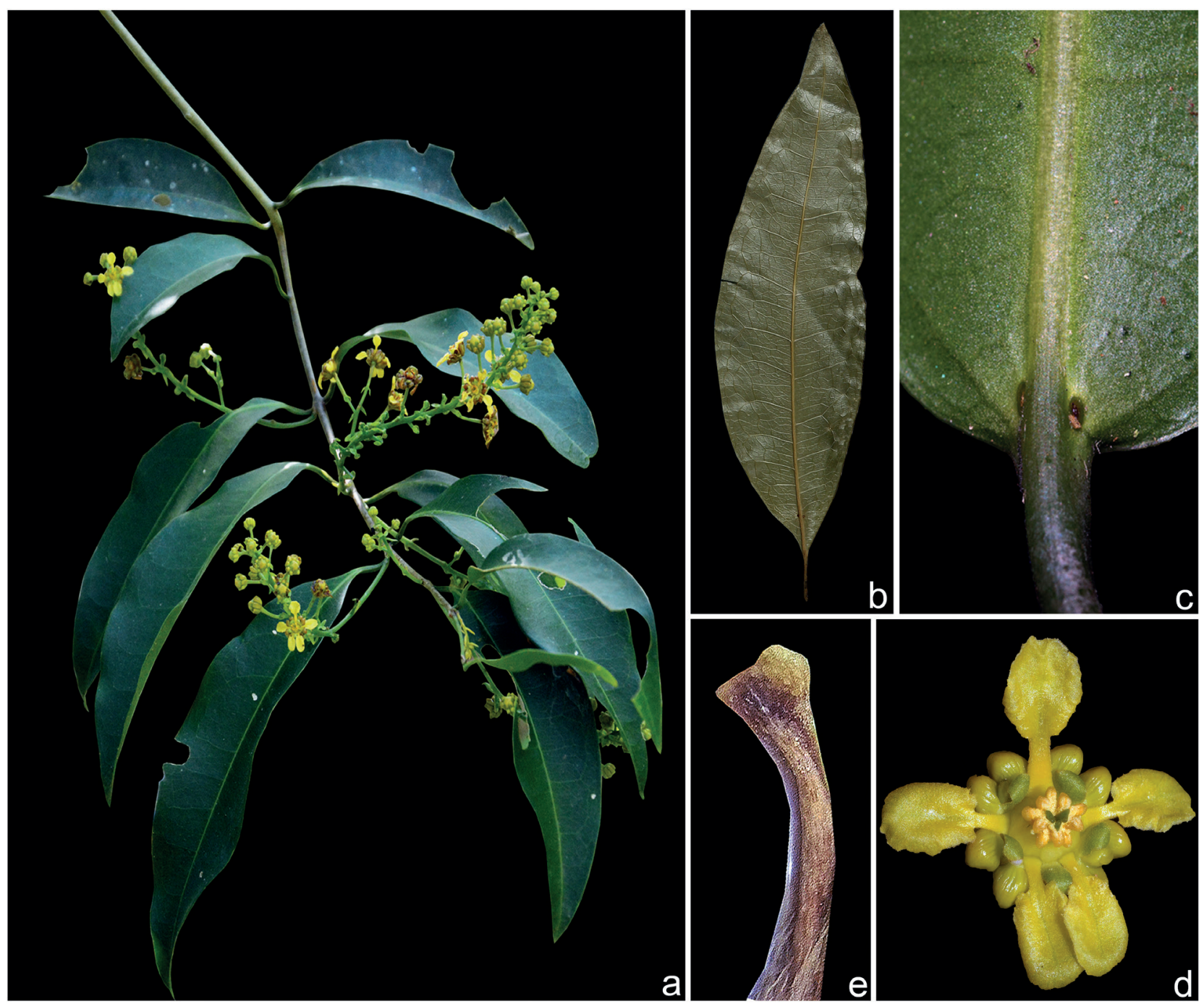

Figure 20. Amorimia andersonii R.F. Almeida: a. flowering branch. b. leaf in abaxial view. c. leaf base showing a pair of glands. d. flower in frontal view. e. detail of the apex of a style (photographs a, c and d by F. Michelangeli, b and e by R.F. Almeida). 
with a pair of glands near base or near margin, patent lateral petals at anthesis, eglandular posterior petals, and anthers pubescent at apex, connectives and base. However, $A$. andersonii can be easily differentiated from $A$. pellegrinii and $A$. rigida by its non-bullate, velutine leaf blades, with 10-16 pairs of right-angled secondary veins, reflexed bracteoles, sepals erect at anthesis, petal claws adaxially velutine, overlapping anterior-lateral petals, filaments pubescent at apex, uncinate style apex, and samaras with depressed ovate dorsal wings.

\subsection{Amorimia candidae R.F.Almeida, Phytotaxa} 284(1): 8. 2016. Holotype: BRAZIL. Bahia: Mun. Itaberaba, margens da rodovia BR-242, 16-VII-2013, fl. fr., R.F. Almeida, M. Alves, L.M.M. Conti, E.C. Chagas \& C.F. Hall 594 (HUEFS!; isotypes: CEPEC!, $\mathrm{MICH}$ !, NY!, P!, RB!, US!).

Figures 21-22
Woody vines to scandent shrubs; branches striated, sparsely lenticellate, lenticels brown, sparsely sericeous-velutine to glabrous at age; stipules 0.4-0.5 mm long, triangular, glabrous, interpetiolar on branches, epipetiolar on inflorescences, persistent to deciduous. Leaves opposite, reduced in inflorescences; petioles 2-5 mm long, canaliculate, sparsely sericeousvelutine to glabrous, eglandular at apex; leaf blades 3-7.7 $\times 1.8-4.4 \mathrm{~cm}$, conduplicate, not bullate, ovate, widely elliptic to orbicular (frequently when associated to the inflorescence), base cordate to rounded, margin revolute, apex mucronate, rounded to retuse, both sides glabrous, 1-2 pairs of glands near base or up to $3 \mathrm{~mm}$ of margins on the distal half, 0.4-0.5 mm diam.; midvein adaxially impressed, abaxially prominent, 5-7 pairs of secondary veins, adaxially impressed, abaxially reddish, prominent, arching $22-24^{\circ}$, subopposite to alternate, reticulum prominent on both sides. Thyrsi
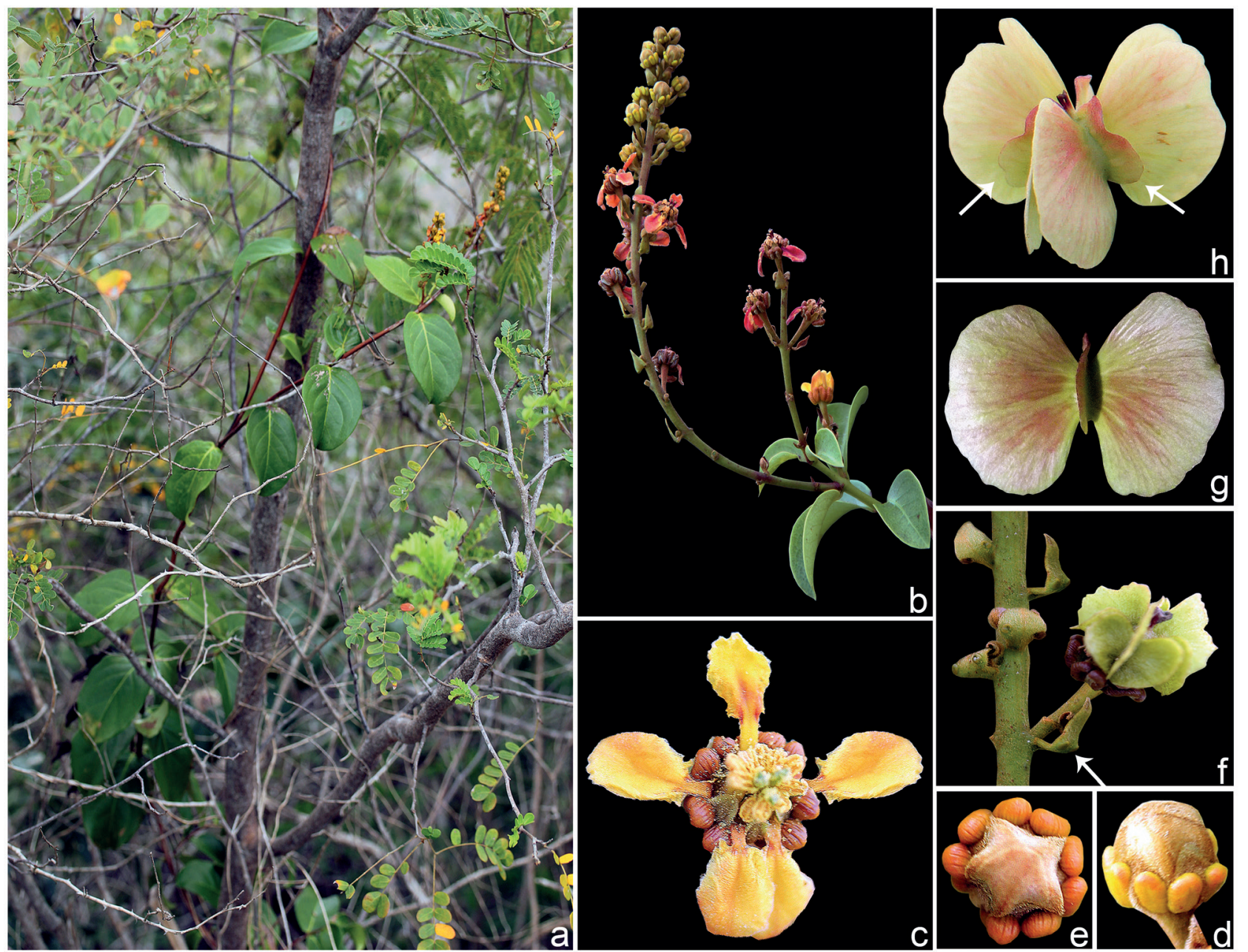

Figure 21. Amorimia candidae R.F. Almeida: a. habit. b. flowering branch showing conduplicate leaves. c. flower in frontal view. d. floral bud in side view. e. floral bud in frontal view. f. detail of a glandular bract. g. samara in dorsal view. h. winged mericarps showing the dorsal wing with rounded angles (white arrows) (photographs by R.F. Almeida). 
(pseudoracemes) or panicles, axillary; main axis 5-24 cm long, cylindrical, striated, sericeous-velutine; cincinni 28-32, 1-flowered, decussate; reduced leaves orbicular, apex rounded to mucronate; bracts 3.5-4 $\times 1.5-2 \mathrm{~mm}$, ovate to elliptic (sometimes leaflike), plane, sessile, parallel to the peduncle, 1 pair of glands at base, both sides sericeous-velutine; peduncle
1-1.5 $\times 0.9-1 \mathrm{~mm}$, cylindrical, sericeous-velutine; bracteoles 1.5-2 × 1.4-1.5 mm, elliptic, concave, inserted at the apex of peduncles, opposite, parallel to the pedicel, eglandular, both sides sericeousvelutine. Flowers 9-11 mm diam. at anthesis; floral buds 3.5-4.5 × 3.5-4 $\mathrm{mm}$ at anthesis; pedicels 3.5-4 $\times 0.9-1 \mathrm{~mm}$, cylindrical, sericeous-velutine. Sepals
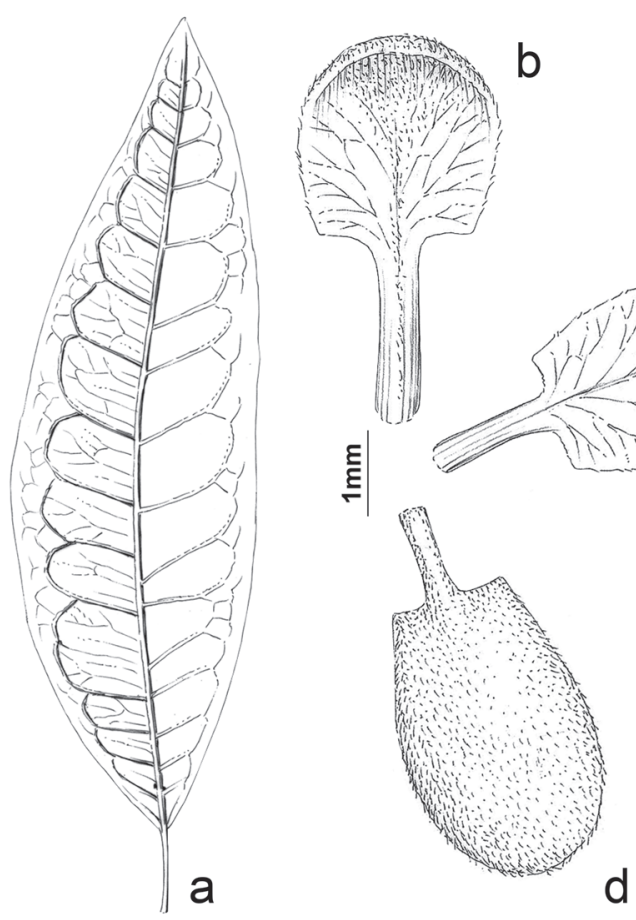

d

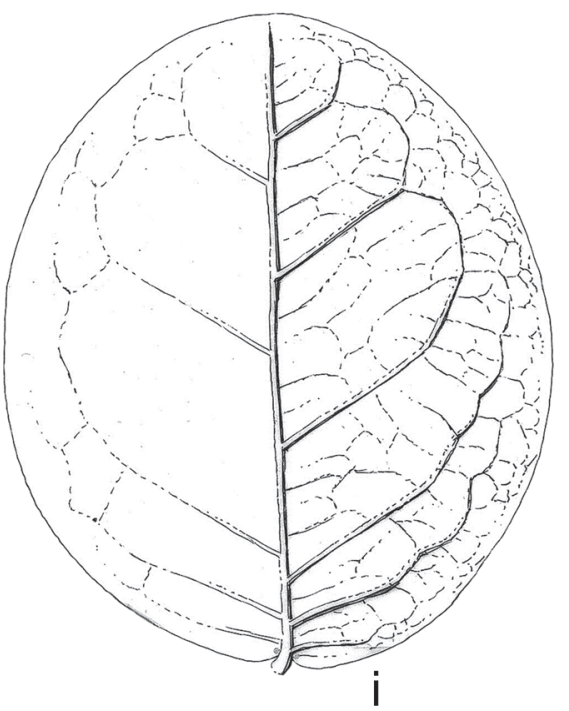

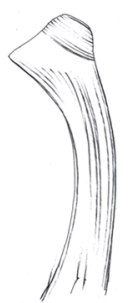

$\mathrm{h}$

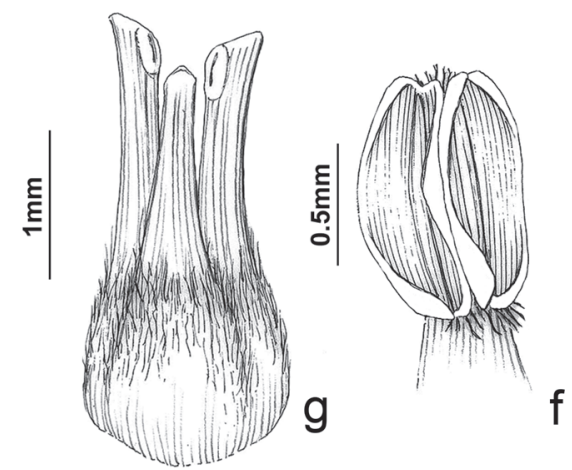

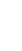
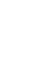
1.8-2 $\times 1-1.5 \mathrm{~mm}$, ovate, appressed to the androecium, apex obtuse to rounded, straight at anthesis, both sides sericeous-velutine; glands yellow turning orange to dark-orange at age, 1.8-2 $\times 0.7-0.8 \mathrm{~mm}$. Petals golden yellow turning red at age, margin sinuate, anteriorlateral petals overlapping at anthesis; lateral petals patent at anthesis, limb 4.5-5 × 3-3.5 mm, elliptic to obovate, truncate at base, adaxially sericeousvelutine distally, abaxially sericeous-velutine; claws 1.4-1.5 × 0.4-0.5 mm, canaliculate, adaxially glabrous, abaxially sericeous-velutine; posterior petal erect at anthesis, limb 4-4.2 × 3.4-3.5 mm, elliptic, truncate at base, 1-pair of red glands at base, adaxially sericeous-velutine distally, abaxially sericeous-velutine; claw 2.3-2.5 × 0.70-0.75 mm, canaliculate, adaxially glabrous, abaxially sericeousvelutine. Stamens opposite petals longer than those opposite sepals; filaments $2.25-2.5 \times 0.25-0.5 \mathrm{~mm}$, connate $0.9-1 \mathrm{~mm}$ long at base, glabrous; anthers dimorphic, those opposite sepals elliptic, those opposite petals obovate, straight, with a glandular connective, $1-1.25 \times 0.5-0.6 \mathrm{~mm}$, reflexed at anthesis, base, connective and apex pubescent. Ovary 1.3-1.5 × 1.3-1.5 mm, each carpel with primordial dorsal and lateral wings, sericeous-velutine; styles 3 , cylindrical at base and apex, parallel at base, divergent at middle, apex truncate with apiculate angle, rarely geniculate, sericeous-velutine at base, glabrous at middle and apex, anterior style 1.1-1.25 mm long, posterior styles 1.4-1.5 mm long; stigma lateral, discoid. Samaras whitish to reddish in vivo; dorsal wing 6-7 × 3-3.25 mm, obtrapezoidal with rounded angles, margin entire, sinuate, both sides sericeousvelutine; lateral wings $1.7-2.5 \times 1.6-2 \mathrm{~cm}$, flabellate, margin erose, sinuate, upper angle $20^{\circ}$, lower angle $25^{\circ}$ from the nut, both sides sparsely sericeous-velutine; nut 5-5.5 $\times 2.5-3 \mathrm{~mm}$, ovoid, sericeous-velutine; areole 4.5-5 × 2-2.2 mm, elliptic. Seeds 3.6-4 × 2-2.25 mm, testa smooth.

Specimens analyzed: BRAZIL. Bahia: Mun. Ibiquera, I-1980, fr., Pinto 5-1980 (CEPEC, FLOR, HRB, $\mathrm{MG})$; Mun. Itaberaba, pastagem, $12^{\circ} 28^{\prime} \mathrm{S}, 40^{\circ} 18^{\prime} \mathrm{W}$, 15-X-2002, fl., Moura 3 (HUEFS); loc. cit., beira da BR, XI-2015, fr., Marques 13, 14 (BHCB); loc. cit., 8-II-1979, fl., Costa 2124 (ALCB); loc. cit., Fazenda Morro da Pedra, 15-VI-1982, fl., Bastos 266 (BAH, CEPEC, HUEFS, IPA); loc. cit., UEP/ Paraguaçú, 6-VIII-1988, fr., Bastos 818 (BAH); loc. cit., 11-III-1982, fl., Ferreira 233 (BAH, MO); loc. cit., Fazenda Itaberaba, morro de Itaberaba, sítio com pinturas, 12 29'57''S, 4004'56' W, 5-VI-2005, fr., Melo 3936 (CEPEC, HUEFS); loc. cit., margens do Paraguaçu, VI-1973, fl., Pinto s.n. (ALCB1929); Mun. Milagres, caatinga arbustiva, IX-2009, fl., Brito 23 (HUEFS); loc. cit., caatinga arbustiva, XII-2009, fl., Brito 28 (HUEFS); loc. cit., BR-116, embaixo da ponte, 19-VIII-2015, fr., Aona 4208 (HUEFS, HURB); Mun. Rafael Jambeiro, Fazenda Coqueiro Rosarinho, km 29 road BR-242, 2-VII-2002, fl., Santos s.n. (CEPEC, HUEFS64423); loc. cit., rio do Peixe, km 22 da BR-242, 11-V-1975, fl., Barroso s.n. (ALCB1930); loc. cit., km 30 da BR-242, 11-V-1975, fl., Barroso s.n. (ALCB1937); Mun. Santa Terezinha, Serra da Jibóia, 12॰47'46”S, 39³1'37'”W, 303 m, 9-X-2010, fl., Melo 8557 (HUEFS). loc. cit., 1247'46”'S, 39³1'37'”W, 303 m, 9-X-2010, fl., Melo 8563 (HUEFS); loc. cit., V-1958, fl., Pinto s.n. (ALCB17936); loc. cit., Serra do Leão, Fazenda Limeira, 12-II-2016, fr., Costa 1630 (HURB); loc. cit., represa da cidade, $12^{\circ} 39^{\prime} 53^{\prime}$ 'S, 39³5'54'’W, 4-IX-2016, fr., Costa 1998, 2006 (HURB, SP).

Distribution, habitat and phenology: Amorimia candidae is known only from SDTF within Caatinga vegetation in Bahia State, Brazil (figure 19). Flowering from July to December. Fruiting from June to July.

Conservation status: Amorimia candidae is represented by only few records restricted to five municipalities, AOO of approximately $20.000 \mathrm{~km}^{2}$ in anthropically modified Caatinga vegetation. Thus, this species should be regarded as Critically Endangered [CR, A2ab; B1b(ii,iii,v); C2a(i); D2; E], due to its EOO being less than $100 \mathrm{~km}^{2}$ and continually declining habitat quality.

Etymology: The epithet honors the Brazilian botanist Dr. Maria Candida Henrique Mamede (b. 1956), colleague, former advisor, and longtime contributor in the studies of Brazilian Malpighiaceae.

Taxonomy notes: Amorimia candidae is similar to $A$. pellegrinii and $A$. rigida, due to its sericeous-velutine leaf blades, with 4-8 pairs of secondary veins, parallel bracteoles, sepals appressed to the androecium, with straight apex at anthesis, elaiophores turning brown at post-anthesis, petal claws adaxially glabrous, patent lateral petals at anthesis, filaments glabrous, anthers pubescent at apex, connectives and base, and styles truncate at apex. Nonetheless, A. candidae can be easily differentiated by its conduplicate, 
non-bullate leaf blades, petals turning red at postanthesis, posterior petal 2-glandular at base of limbs, anterior-lateral petals overlapping, and samaras with obtrapezoidal dorsal wings, with rounded angles.

\subsection{Amorimia coriacea (Griseb.) R.F.Almeida,} Phytotaxa 284(1): 13.2016. Basionym: Mascagnia coriacea Griseb., F1. Bras. 12(1): 92. 1858. $\equiv$ Mascagnia rigida subsp. coriacea (Griseb.) Nied., Arbeiten Bot. Inst. Königl. Lyceums Hosianum Braunsberg 3: 19. 1908. Lectotype (designated by Anderson 2006): BRAZIL. Rio de Janeiro: Woods of Tijuca, XII-1840, fl., G. Gardner 5394 (K barcode 000427423!; isolectotype: BM barcode 000611548!). Figures 23, 25

Woody vines to scandent shrubs; branches striated, sparsely lenticellate, lenticels blackish, sparsely sericeous-velutine to glabrous at age; stipules 0.5-0.6 mm long, triangular, interpetiolar on branches, epipetiolar on inflorescences, persistent to deciduous. Leaves decussate, reduced in inflorescences; petioles 3-5 mm long, canaliculate, sparsely sericeous-velutine to glabrous at age, eglandular to 2-glandular at apex; leaf blades 3-9 × 2-4.5 cm, conduplicate, not bullate, elliptic to ovate, base cuneate, margin plane, apex acute to acuminate, both sides glabrous, eglandular to 1-2 pairs of glands, $0.5-1 \mathrm{~mm}$ diam., at base or distally, up to ca. $3 \mathrm{~mm}$ from the margin; midvein adaxially impressed, abaxially prominent, secondary veins 5-7 pairs, arching $40-50^{\circ}$, subopposite to alternate, adaxially impressed, abaxially prominent, reticulum prominent on both sides. Thyrsi (pseudoracemes) or panicles, terminal; main axis 9-19 cm long, cylindrical, smooth, sericeous-velutine; cincinni $25-40$, 1-flowered, alternate to decussate; reduced leaves elliptic, apex acute; bracts 1.9-2.1 × 1.4-1.5 mm, ovate, plane, sessile, parallel to the peduncle, 1 pair of glands at base, both sides sericeous-velutine; peduncle 4-5 × 0.5-0.7 mm, cylindrical, sericeousvelutine; bracteoles 1.7-2 × 1.5-1.75 mm, wideelliptic, concave, inserted at the apex of the peduncle, parallel to pedicel, 1-2 pairs of glands at base, both sides sericeous-velutine. Flowers 9-12 mm diam. at anthesis; floral buds $3-4 \times 3.5-4.5 \mathrm{~mm}$ at anthesis; pedicels 4.5-5.5 $\times$ 0.4-0.5 mm, cylindrical, sericeousvelutine. Sepals 1.3-1.5 × 1-1.1 mm, ovate, appressed to the androecium, apex obtuse to rounded, straight to revolute at anthesis, both sides sericeous-velutine; glands yellow turning orange to reddish at age, $1.5-2 \times 0.75-1 \mathrm{~mm}$. Petals yellow turning orange to red at age, margin sinuate, anterior-lateral petals not overlapping at anthesis; lateral petals reflexed at anthesis, limb 2.5-4 × 1.5-2 mm, elliptic, base hastate, adaxially sericeous-velutine distally, abaxially sericeous-velutine; claws 0.67-0.75 $\times 0.3-0.4 \mathrm{~mm}$, plane, adaxially glabrous, abaxially sericeousvelutine; posterior petal erect at anthesis, limb 1.5-1.7 × 1.4-1.5 mm, elliptic, base hastate, eglandular, adaxially sericeous-velutine distally, abaxially sericeous-velutine; claw 1.4-1.5 $\times 0.4-0.5 \mathrm{~mm}$, plane, adaxially glabrous, abaxially sericeousvelutine. Stamens opposite petals shorter than those opposite sepals; filaments 1-1.25 × 0.3-0.4 mm, connate $0.15-0.2 \mathrm{~mm}$ long at base, glabrous; anthers dimorphic, those opposite sepals shorter, those opposite petals longer, straight, with a glandular connective, $1-1.25 \times 0.5-0.75 \mathrm{~mm}$, reflexed in anthesis, base pubescent, connective and apex glabrous. Ovary $1-1.25 \times 1-1.25 \mathrm{~mm}$, each carpel with primordial dorsal and lateral wings, sericeous-velutine; styles 3 , cylindrical at base and apex, parallel at base, divergent at middle, apex truncate with obtuse angle, glabrous at base, middle and apex, anterior style 1-1.25 mm long, curved, posterior styles 1.3-1.5 mm long; stigma lateral, crateriform. Samaras green to ocher in vivo; dorsal wing 7.5-8 $\times$ 2.5-3.5 mm, depressed ovate with 1 triangular projection at apex, margin entire, sinuate, both sides sericeous-velutine; lateral wings $1.7-2 \times 1-1.3 \mathrm{~cm}$, flabellate, margin erose, sinuate, upper angle $50-55^{\circ}$, lower angle $60-65^{\circ}$ from the nut, both sides sericeous-velutine; nut 5.2-5.8 $\times 2.5-3 \mathrm{~mm}$, orbicular, sericeous-velutine; areole 6-6.5 $\times 4.5-5 \mathrm{~mm}$, ellipsoid to orbicular. Seeds $4.5-5 \times 3.9-4 \mathrm{~mm}$, testa smooth.

Specimens analyzed: BRAZIL. Rio de JANEIRO: Mun. Armação de Búzios, Área de Proteção Ambiental da Azeda, 2244'44"'S, 4152'09' W, 20-IV-2004, fr., Dantas 610 (HUEFS, RB); loc. cit., Azeda, 22 44'40"S, 41 52 '50"W, 4-IV-2004, fr., Dantas 283 (CEPEC, HUEFS, RB); loc. cit., José Gonçalves, 22 48'00”'S, 41 55'00'”, 20-II-2004, fl., Dantas 176 (HUEFS, RB); loc. cit., Ponta Zapata, 22॰43'00"S, 41 ${ }^{\circ} 57^{\prime} 00$ '”W, 16-II- 2004, fl. fr., Dantas 104 (CEPEC, HUEFS, RB); loc. cit., Ponta do Olho do Boi, 19-I-2000, fl., Fernandes 403 (CEPEC, HUEFS, RB); Mun. Arraial do Cabo, Morro do Miranda, 17-I-2000, fl., Farney 3963 (CEPEC, HUEFS, RB); Mun. Cabo Frio, Morro da Gamboa, próximo ao Bairro da Gamboa, 22 ${ }^{\circ} 53^{\prime}$ S, $42^{\circ} 01^{\prime} \mathrm{W}, 14-\mathrm{III}-2004$, 

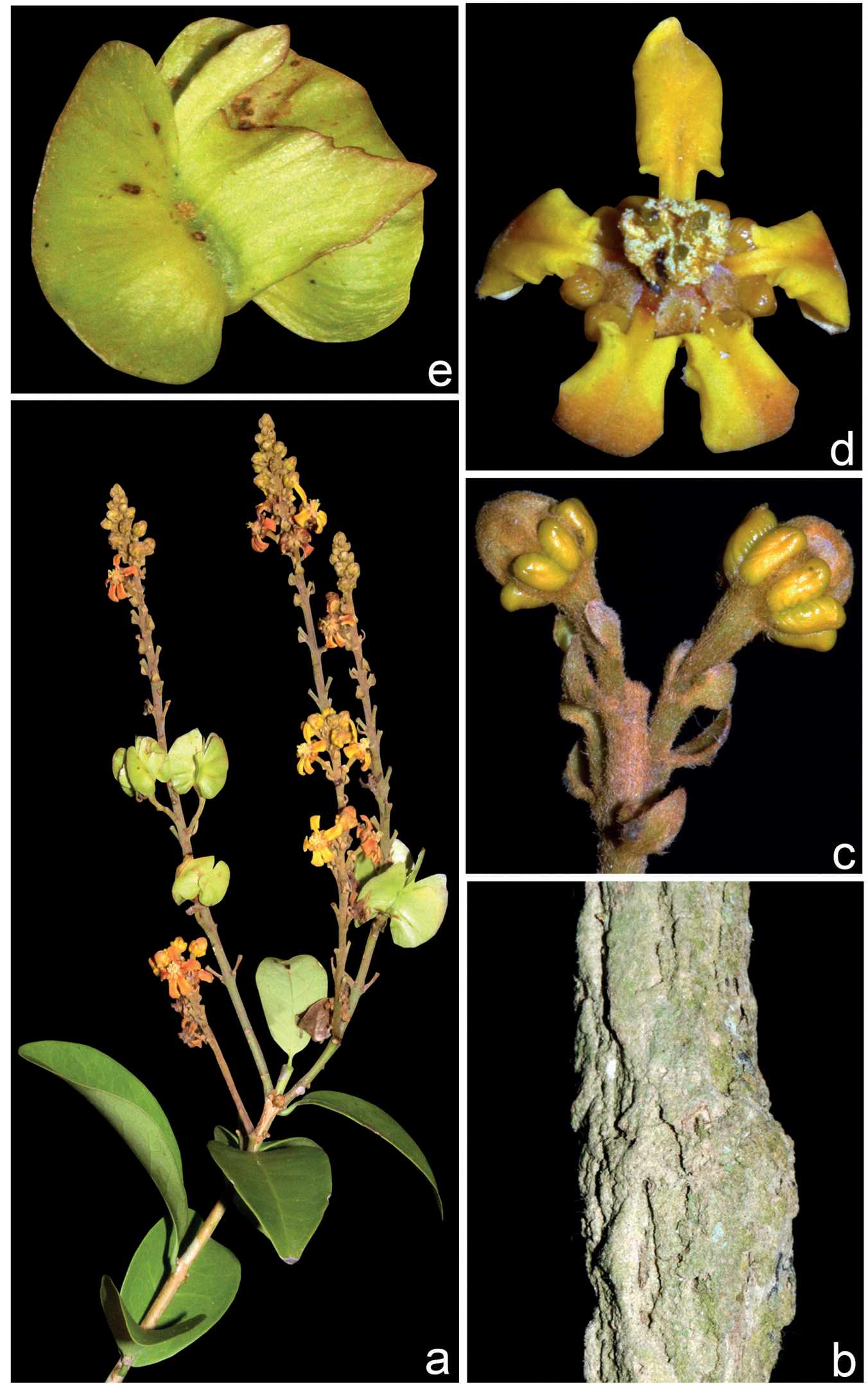

Figure 23. Amorimia coriacea (Griseb.) R.F. Almeida: a. habit. b. stem. c. inflorescence and floral buds in side view. d. flower in frontal view. e. samara in side view (photographs by M.O.O. Pellegrini). 
fl. fr., Jardim 4228 (CEPEC, HUEFS); loc. cit., 20-I-1967, fl., Sucre 1421 (CEPEC, RB, UB); loc. cit., 18-XII-1996, fl., Farag 309 (CEPEC, HUEFS, RB); loc. cit., morro do Mico, 29-III-2007, fl., Farney 4650 (CEPEC, HUEFS, RB); loc. cit., estrada nova para Armação de Búzios, Baia Formosa, entrada para o Capão da Pedra do Sr. Henrique Massala, 6-V-1987, fl. fr., Lima 2877 (CEPEC, HUEFS, RB); loc. cit., Peró, praia das Conchas, 14-I-2016, fl. fr., Almeida \& Pellegrini 615 (CEPEC, HUEFS, RB); Mun. Niterói, Itaipuaçu, Pico Alto Moirão, 17-VI-1985, fr., Andreata 708 (CEPEC, HUEFS, RB); loc. cit., Pico Alto Moirão, 14-I-1982, fl., Andreata 373 (CEPEC, HUEFS, RB); loc. cit., Jurujuba, APA Morro do Morcego, Morro do Pico, 22 57'92,3”S, 4300'49,7”W, 66 m, 15-V-2013, fr., Barros 4909 (RB); Mun. Rio das Ostras, praia, 4-IV-1971, fl., Krieger 10298 (CEPEC, CESJ, HUEFS, MBM). Mun. Rio de Janeiro, Mundo Novo, Botafogo, VI-1921, fl., Kuhlmann 3534 (HUEFS, RB); loc. cit., s.dat., fl., Pohl 5780 (W); loc. cit., s.dat., fl., Schuch 5721 (W68979, W68980).

Distribution, habitat and phenology: Amorimia coriacea is known only from restingas (sand dunes vegetation) and rocky outcrops along the coast of State of Rio de Janeiro, Brazil (figure 19). Flowering from January to June, and fruiting from February to June.

Conservation status: Amorimia coriacea is represented by records restricted to an EOO of approximately $3,620.000 \mathrm{~km}^{2}$ and AOO of $32.000 \mathrm{~km}^{2}$ in anthropically modified restinga vegetation in the State of Rio de Janeiro, Brazil. Thus, it should be regarded as Endangered [EN, B1ab(iii,iv,v); C2a(i,ii); D2], due to its range of distribution being less than $100 \mathrm{~km}^{2}$ and continually declining habitat quality.

Etymology: The epithet probably makes reference to the coriaceous texture of its leaves.

Nomenclatural notes: Mascagnia coriacea was lectotypified by Anderson (2006) based on two sheets from BM and K. However, the type locality from the lectotype at K is cited only as Woods “Tejuco", Brazil. After some research, the author might have mistakenly assumed that the type locality was "Tejuco" (Arraial do Tejuco), a locality currently named Diamantina, in State of Minas Gerais, Brazil. He probably was led to this assumption based on the type locality of Mascagnia rigida, which is somewhere near Northern Minas Gerais (Jequitinhonha municipality). Nonetheless, after analyzing the label on the isolectotype at BM, I observed that it clearly States
Woods Tejuca, State of Rio de Janeiro as the type locality. "Tejuca" is a misspelling of the tupi-guarani word "Tijuca", meaning swampy area. Woods of Tijuca currently comprehends the area of the Tijuca National Park in the municipality of Rio de Janeiro, State of Rio de Janeiro.

Taxonomic notes: Amorimia coriacea is similar to $A$. maritima and $A$. rigida due to its leaf blades with 5-8 pairs of secondary veins, parallel bracts and bracteoles, sepals apressed to the androecium at anthesis, petal claws adaxially glabrous, posterior petals eglandular, anterior-lateral petals divergent, and styles truncate at apex. Nevertheless, $A$. coriacea can be differentiated from $A$. maritima and $A$. rigida by its conduplicate leaf blades, peduncles exceeding the bracts at anthesis, lateral petals reflexed at anthesis, filaments opposite sepals longer than those opposite petals, anthers pubescent only at base, and samaras with dorsal wing obtrapezoidal bearing an aciculate projection at apex.

\subsection{Amorimia exotropica (Griseb.) W.R.Anderson,} Novon 16(2): 180. 2006. Basionym: Mascagnia exotropica Griseb., F1. Bras. 12(1): 93. 1858. Lectotype (designated by Anderson 2006): BRAZIL. Rio Grande do Sul: near Mun. Porto Alegre, s.dat., fl., H. Fox 19 (K barcode 000427411!).

\section{Figures 24-25}

Woody vines to scandent shrubs; branches striated, sparsely lenticellate, lenticels whitish, sparsely sericeous-velutine to glabrous at age; stipules 0.7-1.2 mm long, triangular, interpetiolar on branches, epipetiolar on inflorescences, persistent to deciduous. Leaves opposite, reduced in inflorescences; petioles 8-15 mm long, canaliculate, sparsely sericeous-velutine to glabrous at age, eglandular; leaf blades 5-11.5 × 2-7 cm, plane, not bullate, elliptic to ovate, base rounded to obtuse, margin plane, apex acuminate, both sides glabrous, 1 pair of glands at base, $0.25-0.5 \mathrm{~mm}$ diam., 1-3 pairs of glands up to 1.5-4.0 mm of margins; midvein adaxially impressed, abaxially prominent, 11 pairs of secondary veins, arching $15^{\circ}$, subopposite to alternate, adaxially impressed, abaxially prominent, reticulum prominent on both sides. Thyrsi (pseudoracemes) or panicles, axillary; main axis 6-18 cm long, flattened, irregularly longitudinally costate, sericeous-velutine; cincinni 26-42, 1-flowered, spirally alternate to decussate; reduced leaves elliptic, apex acute; bracts 3-3.5 $\times 1-1.5 \mathrm{~mm}$, ovate to lanceolate, plane, petiolate, parallel to the peduncle, 1-3 pairs of marginal 

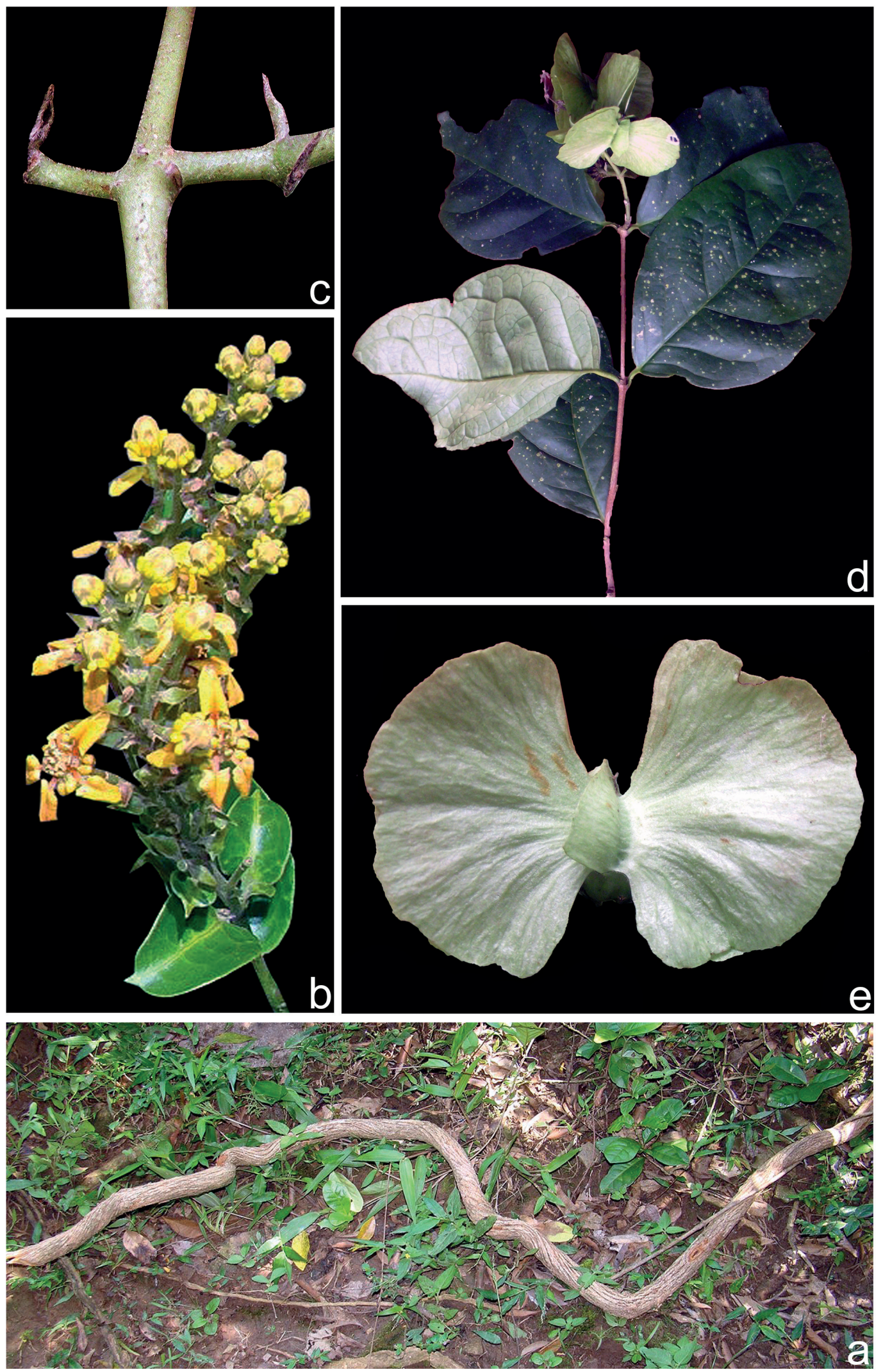

Figure 24. Amorimia exotropica (Griseb.) W.R. Anderson: a. habit and stem. b. inflorescence. c. detail of the inflorescence bracteoles. d. fruiting branch. e. fruit in frontal view (photographs a-b by A. Gava, c-e by R.F. Almeida). 

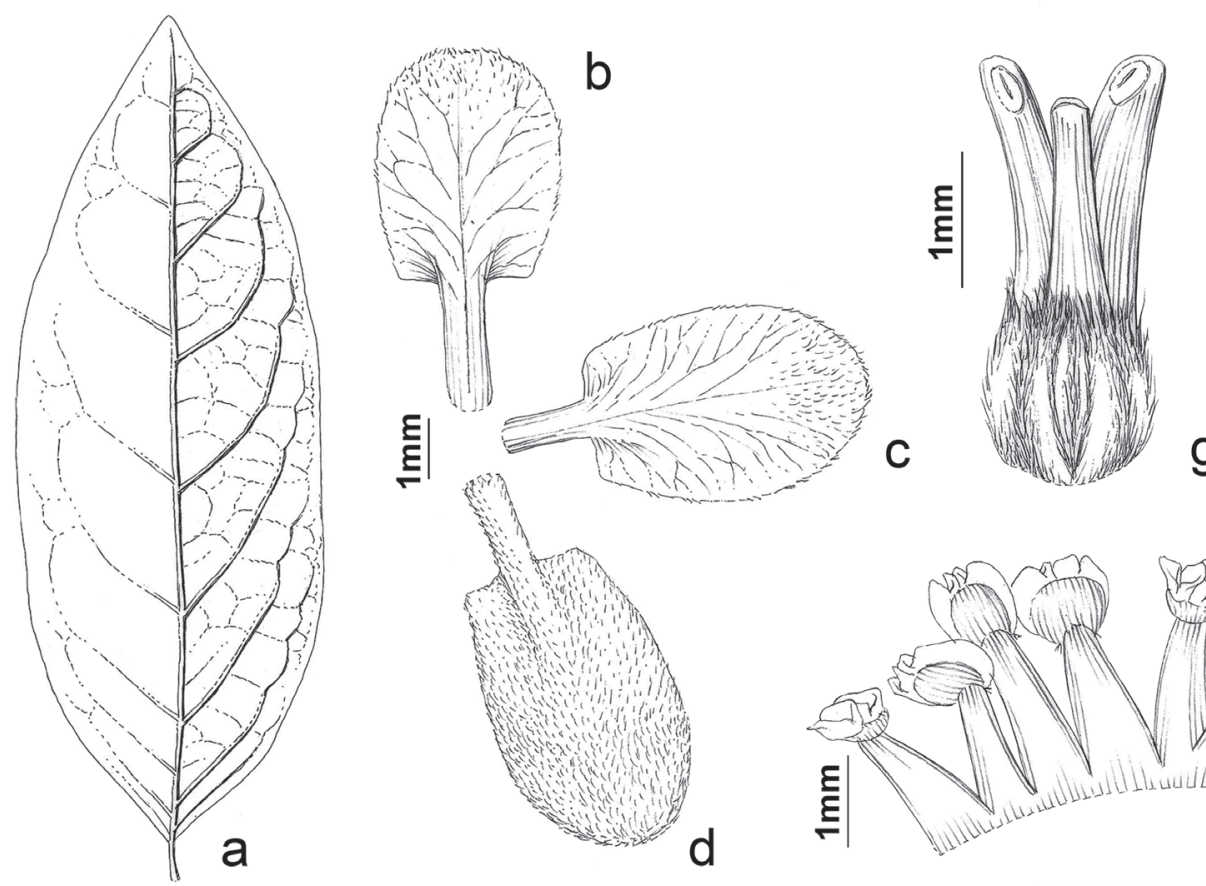

$\frac{1}{201}$
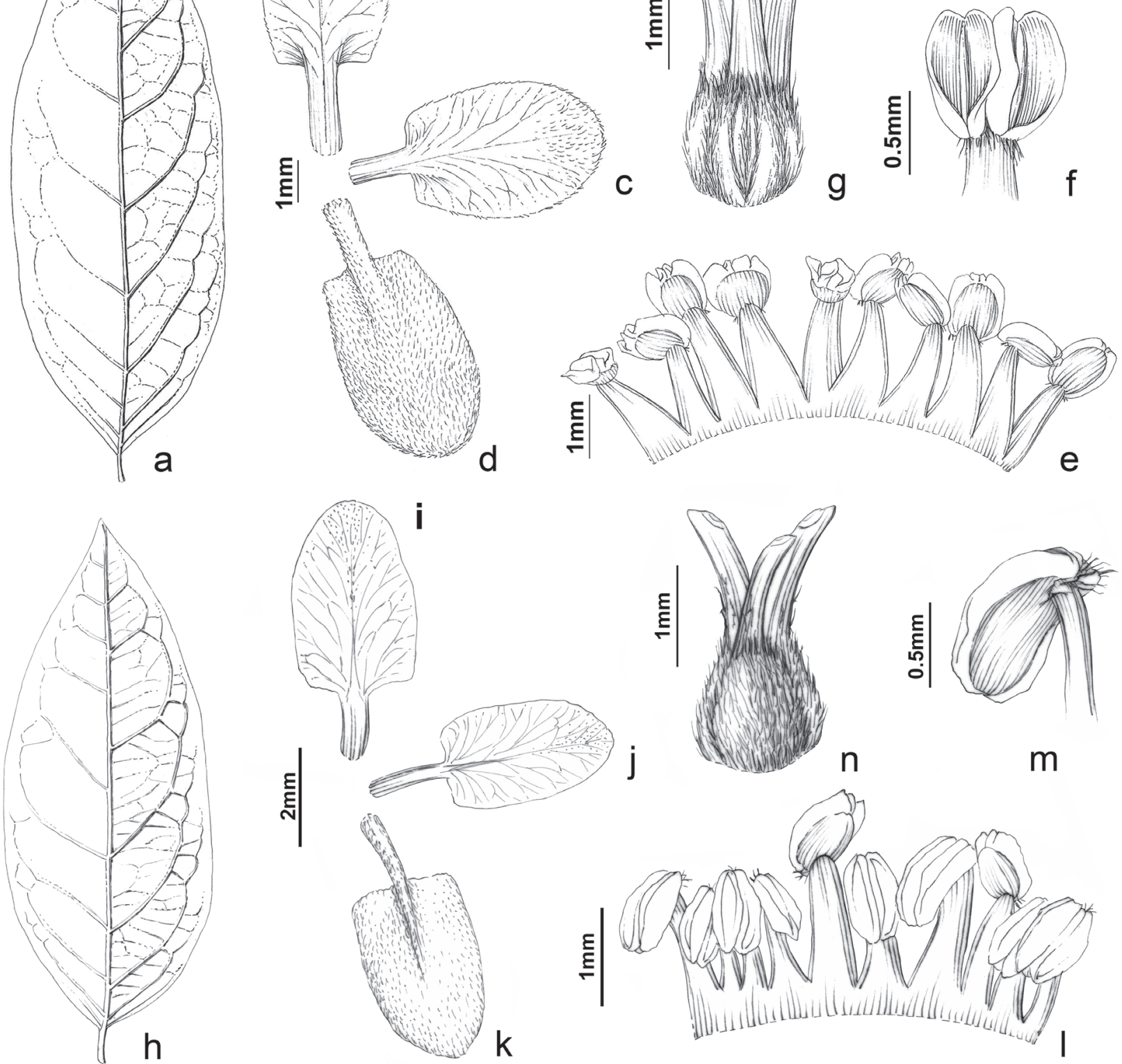

m

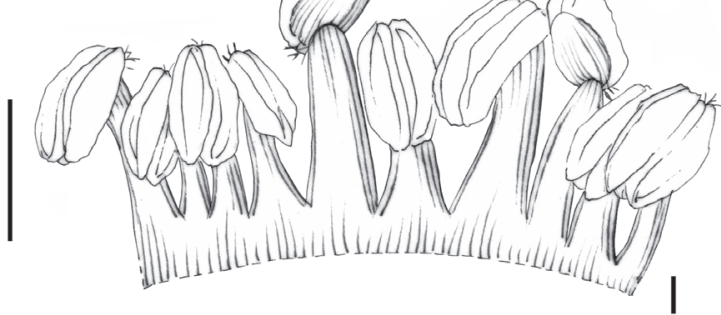

Figure 25. Amorimia coriacea (Griseb.) R.F. Almeida: a. abaxial side of a leaf evidencing vein pattern. b. adaxial side of a posterior petal. c. adaxial side of a posterio-lateral petal. d. abaxial side of a anterio-lateral petal. e. androecium. f. detail of a stamen evidencing anther hairs. g. gynoecium (drawings by Klei Sousa). Amorimia exotropica (Griseb.) W.R. Anderson: h. abaxial side of a leaf showing vein pattern. i. adaxial side of a posterior petal. j. adaxial side of a posterio-lateral petal. k. abaxial side of a anterio-lateral petal. 1. androecium. m. detail of a stamen evidencing anther hairs. n. gynoecium (drawings by Klei Sousa). 
glands, both sides sericeous-velutine; peduncle 2.5-4 × 0.4-0.5 mm, cylindrical, sericeous-velutine; bracteoles $2-3 \times 0.5-1 \mathrm{~mm}$, elliptic to lanceolate, concave, opposite, inserted bellow the apex of peduncles, spreading to the pedicel, eglandular to 1 pair of marginal glands, both sides sericeousvelutine. Flowers $1.2-1.3 \mathrm{~mm}$ diam. at anthesis; floral buds 3.5-4 $\times 3-3.5 \mathrm{~mm}$ at anthesis; pedicels 3.5-7.5 $\times$ 0.5-0.6 mm, cylindrical, sericeous-velutine. Sepals 1-1.5 $\times 0.5-0.75 \mathrm{~mm}$, ovate, appressed to the androecium, apex obtuse to rounded, straight to revolute at anthesis, adaxially sericeous-velutine, abaxially velutine; glands yellow turning orange or red, 2-2.5 $\times 1-1.2 \mathrm{~mm}$. Petals yellow turning orange, margin sinuate, anterior-lateral petals not overlapping; lateral petals patent at anthesis, limb 3.5-4.5 × 2-2.5 mm, elliptic, base truncate, adaxially sericeous-velutine distally, abaxially sericeousvelutine; claws $1.4-1.5 \times 0.45-0.5 \mathrm{~mm}$, plane, adaxially glabrous, abaxially velutine; posterior petal erect at anthesis, limb 3.5-4 × 2.5-3 mm, elliptic, base truncate, eglandular, adaxially sericeous-velutine distally, abaxially sericeous-velutine; claw 1.9-2 $\times 0.5-0.6 \mathrm{~mm}$, plane, adaxially glabrous, abaxially velutine. Stamens opposite petals shorter than those opposite sepals; filaments 1.1-2.5 $\times 0.25 \mathrm{~mm}$, connate ca. $0.5 \mathrm{~mm}$ long at base, glabrous; anthers monomorphic, straight, with a glandular connective, $0.75-1 \times 0.48-0.5 \mathrm{~mm}$, reflexed in anthesis, base, connective and apex pubescent. Ovary 1-1.4 × 1-1.4 mm, each carpel with primordial dorsal and lateral wings, sericeous-velutine; styles 3, cylindrical at base and apex, parallel at base, divergent at middle, apex rounded, base sericeous-velutine, middle and apex glabrous, anterior style $1.25-1.3 \mathrm{~mm}$ long, posterior styles 1.45-1.5 mm long; stigma lateral, crateriform. Samaras green to ocher in vivo; dorsal wing 8-10 $\times$ 4.5-5 mm, trapezoidal with right angles and an aciculate projection at apex, margin entire, sinuate, both sides sericeous-velutine; lateral wings 1.8-2 $\times 1.3-1.5 \mathrm{~cm}$, flabelliform, margin entire, sinuate, upper angle $35^{\circ}$, lower angle $55^{\circ}$ from the nut, both sides sericeous-velutine; nut 7.5-8 $\times 3.5-3.8 \mathrm{~mm}$, ovoid, sericeous-velutine; areole 7.5-8 $\times 3.3-3.5 \mathrm{~mm}$, ovate. Seeds 6-6.5 $\times 3.8-4 \mathrm{~mm}$, testa smooth.

Specimens analyzed: ARGENTINA. Misiones: Dep. Iguazú, Parque Nacional Iguazú, 16-XII-1991, fl., Vanni 2903 (CTES); loc. cit., Cataratas, senderos superiors, 8-XII-1988, fl., Guaglianone 2128 (F, SI); loc. cit., Puerto Bemberg, 15-III-1945, fl., Hayward s.n. (LIL, NY). BRAZIL. Paraná: sin. loc., s.dat., fl., Dusén 14093 (NY); Mun. Adrianópolis, Colônia Tatupeva, 19-IV-1995, fr., Cordeiro 1217 (CEPEC, MBM); Mun. Bocaiuva do Sul, Descampado, 22-IV-1957, fl., Hatschbach 3780 (MBM); Mun. Foz do Iguaçu, Porto Meira, em frente ao portão de entrada do porto, 8-VI-2013, fr., Almeida 549 (FUEL, HUEFS, MBM); loc. cit., 10-III-1980, fl., Buttura 567 (MBM); loc. cit., Parque Nacional do Iguaçu, rio Iguaçu, $25^{\circ} 36^{\prime} 50,7^{\prime} \mathrm{S}, 54^{\circ} 23^{\prime} 36,3^{\prime \prime} \mathrm{W}$, 193 m, 16-X-2015, fl. fr., Caxambu 7035 (HCF); Mun. Guaraqueçaba, Serrinha, 30-50 m, 8-III-1968, fl., Hatschbach 18685 (MBM, MO); loc. cit., Tagaçaba de Cima, 25-IV-1980, fl., Hatschbach 42983 (MBM); Mun. Morretes, Barra do Passa 7, rio Nhundiaquara, 16-I-1976, fl., Hatschbach 37957 (MBM, MO, P); loc. cit., Colônia Floresta, 23-I-1969, fl. fr., Hatschbach 20871 (MBM); Mun. Paranaguá, Ilha do Mel, Praia Grande, Morro do Meio, 24-IV-1987, fl. fr., Britez s.n. (UPCB32213); Mun. Rio Branco do Sul, caverna de Bromado, 25-V-1996, fr., Tiepolo 632 (EFC). Rio Grande do Sul: Mun. Dois Irmãos, XII-1983, fl., Neves 332 (HAS); Mun. Gravataí, Morungava, 9-XII-1983, fl., Mentz s.n. (ICN95118); loc. cit., 27-III-1983, fr., Schenkel s.n. (ICN95120); Mun. Guaíba, Morro José Lutzenberger, 12-IX-2013, fr., Grings 1774 (ICN); Mun. Nova Petrópolis, Panelão, s.dat., fl., Grings 1186 (ICN); loc. cit., Bairro Joaneta, Margem sul da Serra Geral, 15-I-1990, fl., Schlindwein 532 (MPUC, UFP); loc. cit., Bairro Joaneta, Margem sul da Serra Geral, 4-I-1990, fl., Schlindwein 534 (MPUC); Mun. Novo Hamburgo, Ferrabraz, 12-I-1949, fl., Rambo 39952 (PACA); Mun. Osório, Lagoa dos Barros, encosta da serra, 19-XII-1945, fl., Schultz 463 (ICN, HAS); loc. cit., 7-I-1965, fl., Hagelund 3210 (ICN); loc. cit., Maquiné, Estação Experimental Fitotécnica, I-1981, fl., Mattos 22457 (HAS); loc. cit., Morro da Borússia, 23-II-2011, fl., Durigon 570 (ICN); loc. cit., 27-III-1982, fr., Sobral s.n. (MBM73963); Mun. Parecis, Monte Negro, 28-XI-1945, fl. fr., Sehnem 1390 (PACA); loc. cit., 25-I-1946, fl., Trieder 33074 (PACA); loc. cit., 1-VII-1952, fr., Rambo 52768 (PACA); Mun. Parobé, morro do Pinhal, 3-IV-1986, fl., Ritter s.n. (F2111927); Mun. Porto Alegre, morro da Glória, 17-V-2009, fr., Grings 752 (HUCS); loc. cit., Vila Conceição, 28-V-1949, fr., Emrich s.n. (PACA52618, PACA32867); loc. cit., Montserrat, 25-I-1939, fl., Emrich s.n. (PACA26863, PACA30022); loc. cit., Reserva Biológica do Lami, 1-I-2012, fl., Silva-Filho s.n. (MPUC17129); loc. cit., Morro Santa Teresa, 29-IV-1949, fr., Rambo 41303 (L, PACA, W); loc. cit., Belém Novo, 5-III-1944, fr., 
Schultz 61 (ICN); loc. cit., estrada da Ponta Grossa, 20XI-1972, fl., Vianna s.n. (ICN9660, U1367689); Mun. São Francisco de Paula, a $26 \mathrm{~km}$ da cidade, caminho a Taquara, 29-I-1994, fl. fr., Krapovickas 44714 (CTES, U); loc. cit., Fazenda 3 Cachoeiras, I-1999, fl., Senna 135 (HAS); loc. cit., Taquara, a $10 \mathrm{~km}$ de São Francisco de Paula, III-1981, fr., Mattos 22640 (HAS); loc. cit., José Velho, 780 m, 21-I-2001, fl., Wasum 914 (HUCS, MBM); Mun. Torres, Morro Azul, 18-I-1979, fl., Waechter 1176 (ICN); Mun. Viamão, praia do cego, 1-VI-1968, fr., Irgang 384 (ICN). Santa Catarina: Mun. Itapema, IX-1897, fr. Schwacke 12939 (RB); loc. cit., Praia do Cabeço, 10 m, 11-IV-2001, fl., Cervi 8134 (MBM); Mun. Laguna, Morro Nossa Senhora da Glória, 24-I-1984, fl., Krapovickas 39391 (CTES, MBM); Mun. Penha, Morro sobre el mar, coordenadas, 16-I-1985, fl., Krapovickas 39599 (CTES, MO); Mun. Praia Grande, 25-III-2005, fl., Marchett 155 (HUCS, MBM). São Paulo: Mun. Barra do Turvo, Rio do Turvo, 22-II-2009, fl., Ribas 8101 (MBM); Mun. Eldorado, Fazenda Itaipava/Catre, 243'22,7''S, 4806'18,5'’W, 40 m, 27-III-2015, fr., Caxambu 6011 (HCF); Mun. Iguape, Estação Ecológica Juréia-Itatins, Serra da Juréia, trilha do Imperador, 9-XI-1993, fr., Nicolau 1482 (SP); Mun. Peruíbe, Estação Ecológica Juréia-Itatins, Núcleo Guarau, Morro do Fernando, 27-I-2000, fl., Cordeiro 1997 (HUEFS, SP).

Distribution, habitat and phenology: Amorimia exotropica is known to Semi-Deciduous Forests and Rainforests in Southern Brazil, Argentina, and probably Paraguay (figure 19). Flowering from November to February and fruiting from February to August.

Conservation status: Despite possessing a wide EOO (ca. 307,263.546 $\mathrm{km}^{2}$ ), A. exotropica possesses a rather small AOO (ca. $16.000 \mathrm{~km}^{2}$ ). After extensive field trips, I was able to recollect $A$. exotropica in only one of the several visted localities. Most of the visited localities had been greatly affected by human activities, especially rice crops and urban development. Thus, A. exotropica should be regarded as Endangered [EN, B1ab(iii,iv,v); C2a(i,ii); D2].

Etymology: the epithet makes reference to its distribution outside the tropics.

Taxonomic notes: Amorimia exotropica is similar to $A$. coriacea and $A$. maritima due to its elliptic to ovate, sericeous-velutine, non-bullate leaf blades, concave bracteoles, sepals appressed to the androecium, petal claws adaxially glabrous, eglandular posterior petal, posterior petal as long as the lateral petals, and anterior-lateral petals divergent. However, $A$. exotropica can be differentiated by its leaf blades with 11 pairs of secondary veins, bracteoles subopposite and inserted below the apex of the peduncle, petal claws abaxially velutine, anthers pubescent at base, connective and apex, and style apex rounded.

1.5. Amorimia maritima (A.Juss.) W.R.Anderson, Novon 16(2): 181.2006. Basionym: Hiraea maritima A.Juss., Ann. Sci. Nat., Bot., sér. 2 13: 259. 1840. 三 Triopterys polycarpa Salzm. ex A.Juss., Arch. Mus. Hist. Nat. 3: 562 1843. nom. illeg. $\equiv$ Mascagnia rigida subsp. typica Nied.,Arbeiten Bot. Inst. Königl. Lyceum Hosianum Braunsberg 3: 19. 1908. nom. illeg. Holotype: BRAZIL. BAHIA: in petrosis maritimis (rocky coast), s.dat., fl. fr., P. Salzmann s.n. (Gbarcode 00352762!; isotypes: HAL barcode 0118194!, K barcode 000427424 !, K barcode 000427425 !, MO barcode 2155376!, MPU barcode 020107!, MPU barcode 020108!, MPU barcode 020109!, MPU barcode 020110!, MPU barcode 020111!, MPU barcode 020185 !, P barcode 02429158 !, P barcode 02429159!, P barcode 02429160!, P barcode 02429161 !, W315362!).

Figures 26-27

Woody vines to scandent shrubs; branches striated, sparsely lenticellate, lenticels whitish, sparsely sericeous-velutine to glabrous age; stipules 0.5-0.6 mm long, triangular, interpetiolar on branches, epipetiolar on inflorescences, glabrous, persistent to deciduous. Leaves opposite to subopposite, reduced in inflorescences; petioles 7-17 mm long, canaliculate, sparsely sericeous-velutine to glabrous at age, eglandular to biglandular at apex; leaf blades $7-17.7 \times 2.7-8.7 \mathrm{~cm}$, plane, not bullate, elliptic to obovate, base cuneate, margin plane, apex acute to acuminate, both sides glabrous, 1-2 pairs of glands, $0.5-2 \mathrm{~mm}$ diam., near base or up to $4 \mathrm{~mm}$ of margins; midvein adaxially impressed, abaxially prominent, 6-8 pairs of secondary veins, arching $40^{\circ}$, subopposite to alternate, adaxially impressed, abaxially prominent, reticulum prominent on both sides. Thyrsi (pseudoracemes) or panicles, axillary; main axis 5-22.5 cm long, flattened, slightly costate, sericeous-velutine; cincinni 15-30, 1-flowered, alternate; reduced leaves elliptic, apex acute; bracts $2.5-3 \times 1-2 \mathrm{~mm}$, ovate to lanceolate, plane, sessile, parallel to the peduncle, 1-2 pairs of basal glands, both sides sericeous-velutine; peduncle 
1.8-2 $\times$ 0.5-0.6 mm, cylindrical, sericeous-velutine; bracteoles $1.8-2.2 \times 1.25-1.5 \mathrm{~mm}$, elliptic to ovate, concave, inserted at the apex of peduncles, spreading to the pedicel, 1 pair of glands at base or middle, both sides sericeous-velutine. Flowers 1-1.2 mm diam. at anthesis; floral buds $3.5-4 \times 2.8-3 \mathrm{~mm}$ at anthesis; pedicels 3-3.5 $\times 0.5-0.6 \mathrm{~mm}$, cylindrical, sericeous velutine. Sepals 1.25-1.5 × 0.75-1 mm, ovate, appressed to the androecium, apex obtuse to rounded, straight to revolute at anthesis, both sides sericeous-velutine; glands yellow turning orange to reddish, $1.5-2.5 \times 0.75-1 \mathrm{~mm}$. Petals yellow turning red, margin sinuate, anterior-lateral petals not overlapping; lateral petals patent, limb $3.8-4 \times 2-2.25 \mathrm{~mm}$, elliptic, base truncate, adaxially sericeous-velutine distally, abaxially sericeous velutine; claws 1.25-1.5 × 0.25-0.30 mm, plane, adaxially glabrous, abaxially sericeous-velutine; posterior petal erect, limb 4-4.3 × 2-2.2 mm, elliptic, base truncate, eglandular, adaxially sericeousvelutine distally, abaxially sericeous velutine; claw 2-2.2 $\times 0.5-0.54 \mathrm{~mm}$, plane, adaxially glabrous, abaxially sericeous-velutine. Stamens opposite petals longer than those opposite sepals; filaments $1.25-2 \times 0.25-0.5 \mathrm{~mm}$, connate ca. $0.5 \mathrm{~mm}$ at base, sericeous at apex; anthers dimorphic, those opposite
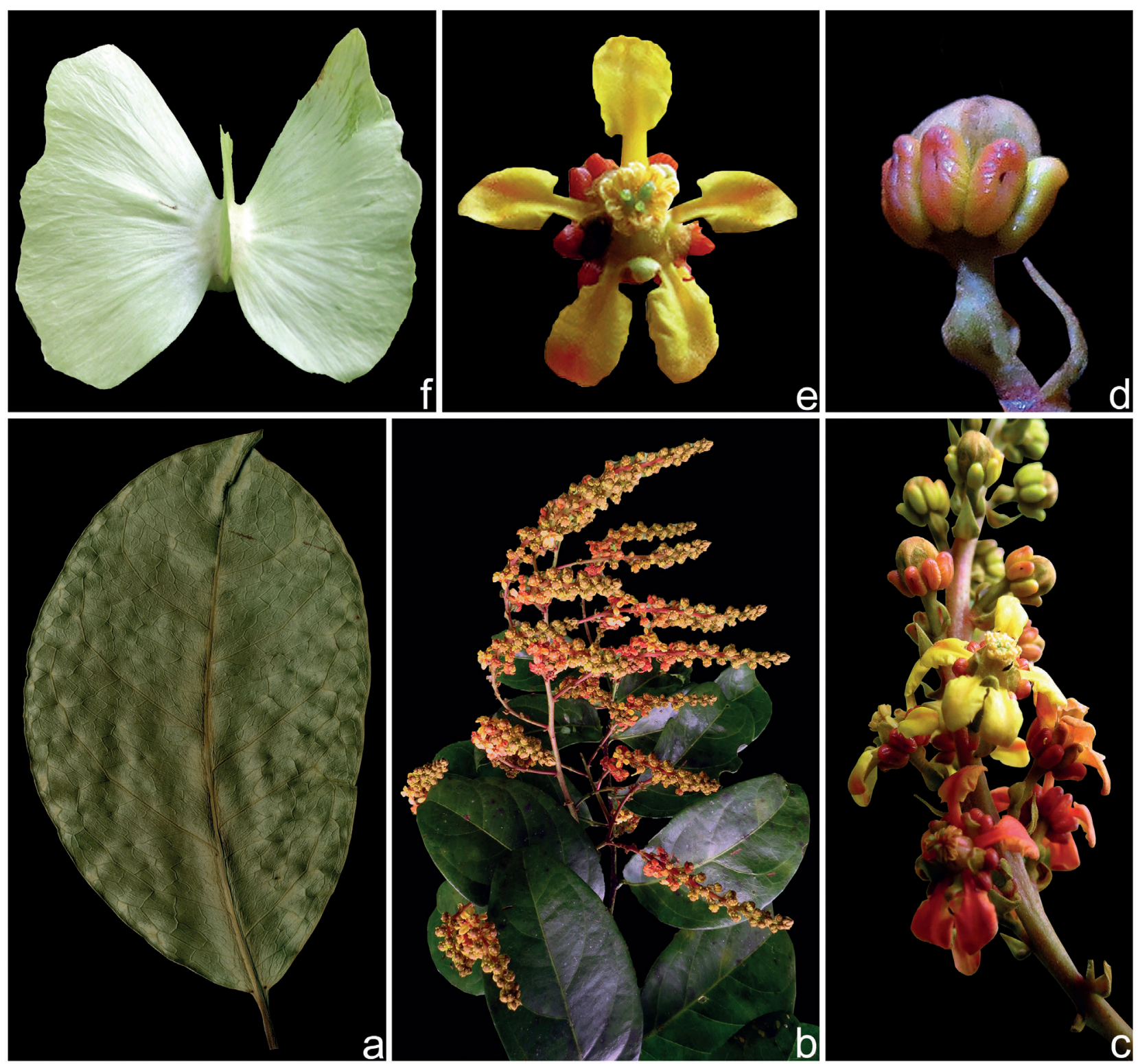

Figure 26. Amorimia maritima (A. Juss.) W.R. Anderson: a. leaf in abaxial view. b. flowering branch. c. detail of an inflorescence and flowers. d. floral bud in side view. e. flower in frontal view. f. samara in frontal view (photographs a, f by R.F. Almeida, b-e by F. Flores). 

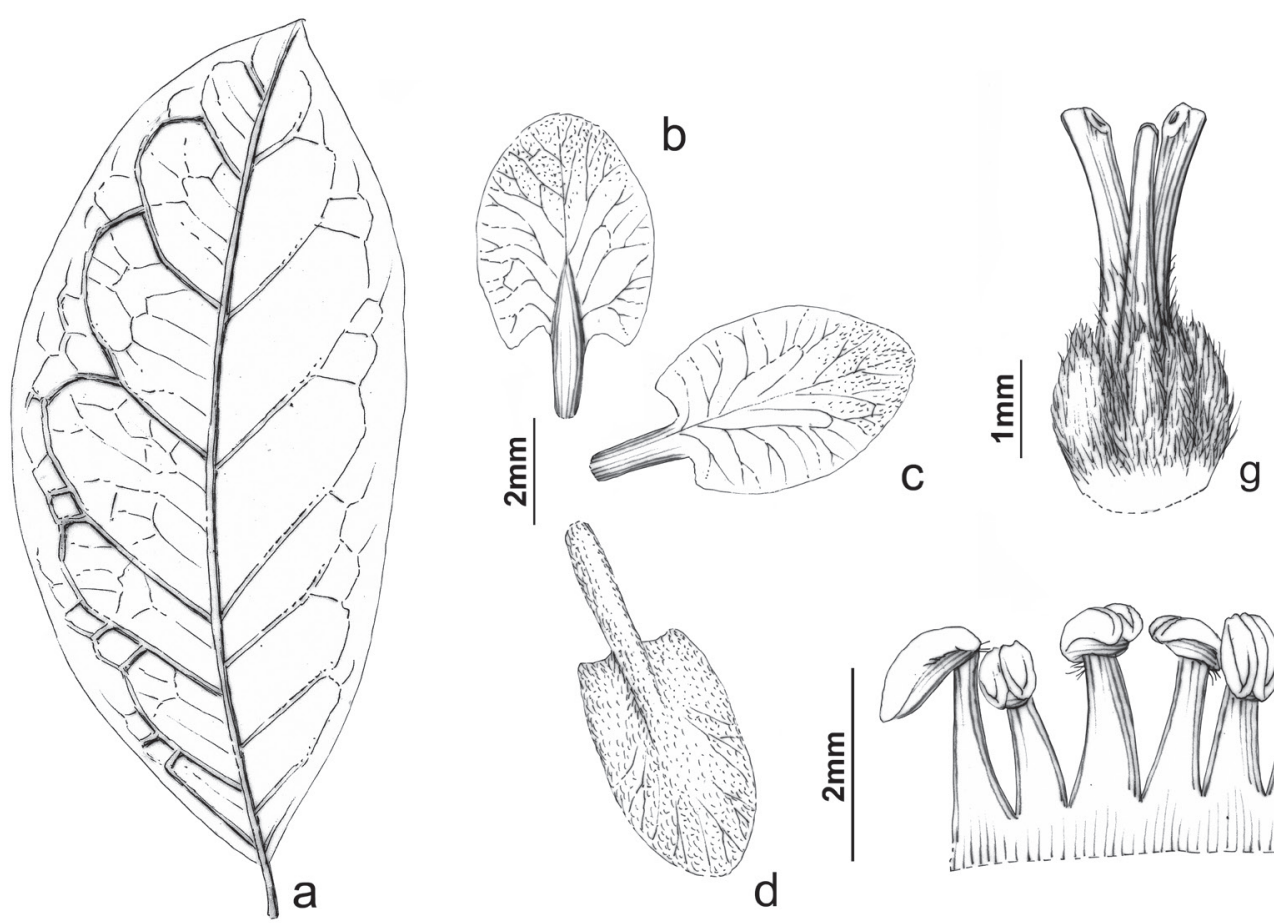

留
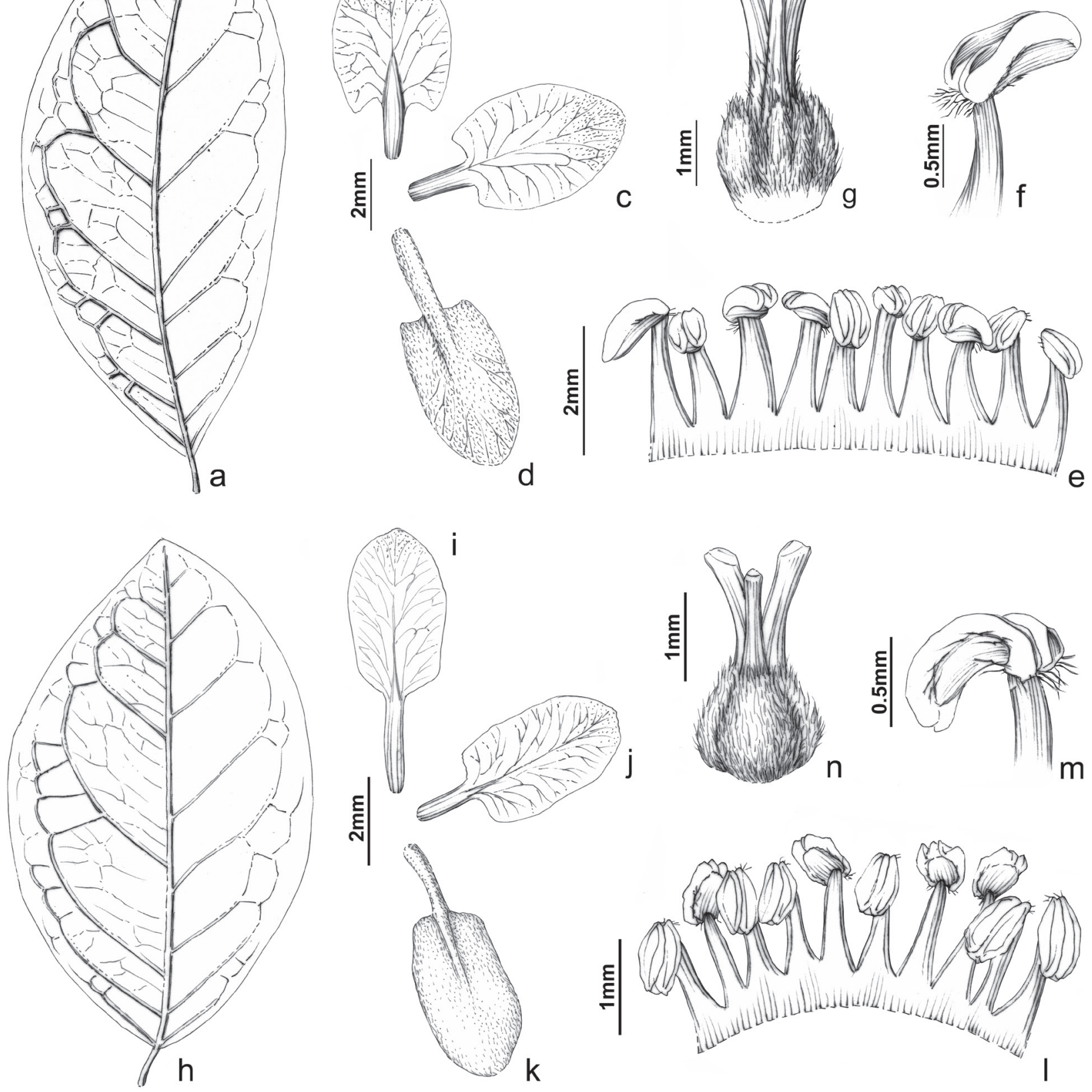

\section{.}

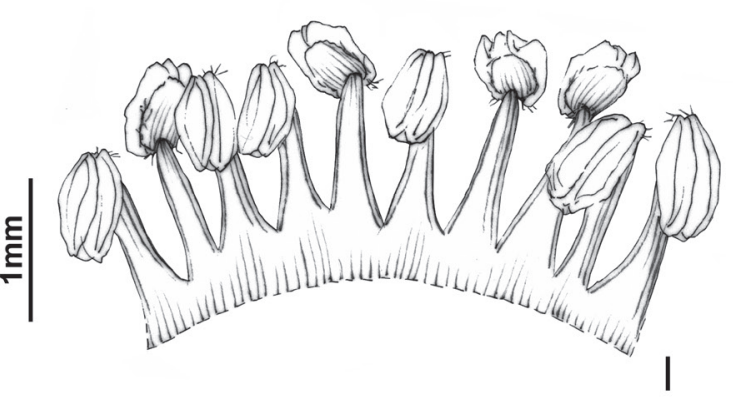

Figure 27. Amorimia maritima (A. Juss.) W.R. Anderson from state of Bahia: a. abaxial side of a leaf evidencing vein pattern. b. adaxial side of a posterior petal. c. adaxial side of a posterio-lateral petal. d. abaxial side of an anterio-lateral petal. e. androecium. f. detail of a stamen evidencing anther hairs. g. gynoecium (drawings by Klei Sousa). Amorimia maritima (A. Juss.) W.R. Anderson from state of Espírito Santo: h. abaxial side of a leaf evidencing vein pattern. i. adaxial side of a posterior petal. j. adaxial side of a posterio-lateral petal. k. abaxial side of a anterio-lateral petal. 1. androecium. m. detail of a stamen showing anther hairs. n. gynoecium (drawings by Klei Sousa). 
petals narrower than those opposite sepals, straight, with a glandular connective, $0.75-1.25 \times 0.5-0.55 \mathrm{~mm}$, reflexed in anthesis, pubescent at base and apex. Ovary 1-1.5 × 1-1.5 mm, each carpel with primordial dorsal and lateral wings, sericeous-velutine; styles 3 , cylindrical at base and apex, parallel at base, divergent at middle, apex truncate with obtuse angle, sericeous-velutine at base, glabrous at middle and apex, anterior style 1.5-2.0 mm long, curved, posterior styles $1.75-2.25 \mathrm{~mm}$ long, erect; stigma lateral, discoid. Samaras bright green turning reddish in vivo; dorsal wing 8-13 $\times 5-7 \mathrm{~mm}$, trapezoidal with right angles and a aciculateprojection at apex, margin entire, sinuate, both sides sericeous-velutine; lateral wings 1.8-3.8 $\times 1.1-2.5 \mathrm{~cm}$, flabelliform, margin erose, sinuate, upper angle $40-50^{\circ}$, lower angle $50-60^{\circ}$ from the nut, both sides sericeous-velutine; nut 6-8.5 $\times 3-5 \mathrm{~mm}$, orbicular, sericeous-velutine; areole 6-9.5 $\times$ 4-6.5 mm, ellipsoid to orbicular. Seeds 4.5-5 $\times 3-3.5 \mathrm{~mm}$, testa smooth.

Specimens analyzed: BRAZIL. BAHIA: sin. loc., s.dat., fl., Blanchet s.n. (P04843525); sin. loc., 1833, fl., Blanchet 1660 (F, G); sin. loc., 1834, fl., Blanchet 1720 (3 ex F, 3 ex G); Mun. Barro Preto, estrada para Pedra Lascada, $12 \mathrm{~km}$ após a saída da cidade, 5-VI-2013, fl., Pace 350 (HUEFS, SPF); loc. cit., Serra da Pedra Lascada, $13,7 \mathrm{~km}$ da cidade, entrada de acesso pela Fazenda São Miguel, 1446'13' S, 39¹2'10'”, 600-900 m, 26-IV-2004, fl., Amorim 4102 (CEPEC); loc. cit., estrada para Pedra Lascada, XI-2015, fr., Marques 5 (BHCB); Mun. Candeias, Fazenda Mariza, V-2004, fl., Reis s.n. (CEPEC99176); Mun. Ilhéus, ramal que separa a Fazenda Alegrias do Campus da Universidade Estadual de Santa Cruz, 7-VI-1995, fl., Mattos Silva 3136 (ALCB, CEPEC, HUEFS, HUESC); loc. cit., Castelo Novo, XII-1821, fl., Riedel 503 (K); loc. cit., $\mathrm{km} 22$ da rodovia Ilhéus-Itabuna (BR-415), Área do Centro de Pesquisas do Cacau, quadra D, 2-VI-1982, fr., Santos 21 (CEPEC); loc. cit., 24VIII-1991, fl., Amorim 319 (CEPEC, NY); loc. cit., 3-IX-1998, fr., Amorim 2483 (CEPEC, RB); Mun. Itabuna, rodovia BR-415, sentido Itabuna-Ibicaraí, ca. $5 \mathrm{~km}$ de Ferradas, margem da rodovia, $15^{\circ} 12^{\prime} 10^{\prime}$ 'S, 39²7'40"W, 19-VIII-2008, fl., Jardim 5350 (CEPEC, HPL); loc. cit., saída para Uruçuca, 15-V-1968, fl., Belém 3551 (IAN, NY); Mun. Jussari, RPPN Serra do Teimoso, $15^{\circ} 10^{\prime}$ S, 39³5'W, 1-IX-2001, fr., Oliveira 751 (CEPEC, HUEFS); loc. cit., Rodovia JussariPalmira, entrada ca. $7.5 \mathrm{~km}$ de Jussari, $15^{\circ} 09^{\prime} 16^{\prime \prime} \mathrm{S}$, 39³1'52"W, 641 m, 13-VI-2004, fl., Amorim 4127
(CEPEC, HUEFS, NY, SP); Mun. Salvador, Região Metropolitana de Salvador, Ilha de Maré, Povoado de Botelho, 1247'05'S, 38³1'W, 13-VI-2011, fl., Guedes 18404 (ALCB); loc. cit., Ondina, 8-VII-1954, fl., Espinosa 901 (BAH, RB). Espírito Santo: Mun. Alegre, São João do Norte, 17-III-2009, fl., Couto 1130 (MBML, SP, VIES); Mun. Cachoeiro do Itapemirim, Sítio do Remy, 4-XI-1991, fl., Vinha 1204 (CEPEC, VIES); Mun. Colatina, VI-1984, fr., Döbereiner \& Tokarnia 1765 (NY); Mun. Linhares, Bebedouro, 26VIII-1981, fl., Döbereiner \& Tokarnia 1677 (CEPEC, R, RB, UB); Mun. Mimoso do Sul, Fazenda Gabiroba, 21 ${ }^{\circ} 12^{\prime} 36^{\prime}$ 'S, 41 ${ }^{\circ} 21^{\prime} 26^{\prime}$ 'W, 36 m, 2-IV-2001, fl., Pereira 37-84 (RFA); Mun. Nova Venécia, APA Pedra do Elefante, trilha principal na mata da Fazenda Santa Rita, 18 46'57'S, 40²5'58'W, 154 m, 17-VII-2008, fr., Amorim 7512 (CEPEC, RB); loc. cit., APA Pedra do Elefante, Serra de Baixo, $18^{\circ} 46^{\prime} 01$ ' S, 40²7'28”W, 653 m, 10-V-2008, fl., Amorim 7425 (CEPEC, MBML, RB, UPCB); loc. cit., APA Pedra do Elefante, Serra de Baixo, 1846’01''S, 40²7’28”W, 653 m, 10-V-2008, fl., Amorim 7426 (CEPEC, MBML, RB, UPCB); loc. cit., APA Pedra do Elefante, 18 46’37'S, 40²6’38”W, 400 m, 17-VII-2008, fl., Amorim 7528 a (CEPEC, MBML, RB, UPCB); loc. cit., APA Pedra do Elefante, 1846’37'S, 40²6’38'W, 400 m, 17-VII-2008, fr., Amorim 7529 (CEPEC, MBML, RB, UPCB); loc. cit., APA Pedra do Elefante, Serra de Baixo, Mata da Fazenda Santa Rita, 1846'40'S, 40²6'40"W, 154 m, 15-IV-2009, fr., Forzza 5534 (CEPEC, MBML, RB, UPCB); loc. cit., Serra de Cima, Torre 101/1, 30¹8'49.9"'S, 40²8'46.8"W, 120 m, 25-IV-2008, fr., Assis 1522 (MBML, SP); Mun. Santa Teresa, Pedra da Onça, propriedade de Antonio Rocon, 13-VI-2000, fr., Demuner 1101 (CEPEC, HUEFS, MBML); loc. cit., Escola Agrotécnica, Federal, 12-V-1999, fr., Lopes 698 (HUEFS, MBML); loc. cit., Várzea Alegre, mata do sr. Fausto, fundos do patrimônio, 1953'42"'S, 4045'46" W, 244 m, 30-I-2002, fl., Groppo Jr. 983 (CEPEC, SP, SPF); loc. cit., rio Saltinho, beira da estrada Fundão-Santa Teresa, 29-V-2001, fr., Kollmann 3726 (CEPEC, MBML, SP); Mun. São Mateus, 24-V-1998, fr., Döbereiner \& Tokarnia s.n. (NY01018898); loc. cit., 19-VI-1999, fr., Döbereiner \& Tokarnia s.n. (NY01018899); loc. cit., Fazenda Pedra Linda, pasto cachoeira, 16-VI-1984, fl., Döbereiner \& Tokarnia 1771 (CEPEC, MICH, NY, RB); loc. cit., Reserva Biológica do Córrego Grande, 18-VI-1984, fl. fr., Döbereiner \& Tokarnia 1773 (CEPEC, K, R, RB); loc. cit., Fazenda Boa Lembrança, 19-VI-1984, fl. fr., 
Döbereiner \& Tokarnia 1774 (CEPEC, K, R, RB); loc. cit., Reserva Biológica de Sooretama, lagoa do Macaco, 15-V-1977, fl., Martinelli 2232, 2240 (RB); Mun. Serra, APA Mestre Álvaro, 15-II-2013, fl., Barros 160 (HUEFS, VIES); Mun. Sooretama, Reserva Biológica de Sooretama, próximo a ponte do córrego Rodrigues, 13-VIII-2010, fr., Siqueira 561 (CEPEC, CRVD). Rio de Janeiro: Mun. Cambuci, três irmãos, 9-V-1919, fr., Sampaio 3219 (R); Mun. Campos dos Goytacazes, Morro do Itaoca, trilha pra torre, 22-I-2010, fl., Mauad 128 (RB, UEHF); Mun. Itaocara, ilhas fluviais do rio Paraíba, ilha do Santíssimo, 22-V-2004, fr., Vieira 1401 (RB, RFA); Mun. Volta Redonda, Área de Relevância Ecológica da SEMA, Floresta da Cicuta, 13-VIII-1987, fr., Lima 154 (HUEFS, HRB, RB).

Distribution, habitat and phenology: Amorimia maritima is known only to coastal lowland seasonal and rainforests in the States of Bahia, Espírito Santo and Rio de Janeiro, Brazil (figure 19). Flowering and fruiting throughout the year.

Conservation status: despite possessing a wide EOO (ca. $\left.113,060.191 \mathrm{~km}^{2}\right)$, A. maritima possesses a rather small $\mathrm{AOO}\left(\mathrm{ca} .32 .000 \mathrm{~km}^{2}\right)$. Amorimia maritima is exclusive to the Central Corridor of the Atlantic Forest, one of the most greatly threatned portions of this devastated domain (CEPF 2011). Thus, A. maritima should be regarded as Endangered [EN, B1ab(iii,iv,v); C2a(i,ii); D2].

Etymology: the epithet makes reference to its restricted occurrence to coastal forests, usually at sea level.

Taxonomic notes: Amorimia maritima is similar to $A$. pellegrinii and $A$. rigida due to its plane, sericeousvelutine, elliptic to ovate leaf blades, parallel bracteoles, sepals appressed to the androecium, petal claws adaxially glabrous, patent lateral petals, and styles with truncate apex. However, A. maritima can be easily differentiated by its bullate leaf blades, elaiophores turning orange to red at post-anthesis, petals turning red at post-anthesis, eglandular posterior petal, anterior-lateral petals divergent, filaments sericeous at apex, anthers pubescent only at base and apex, and dorsal wing trapezoidal with right angles and a aciculateprojection at apex.

1.6. Amorimia pellegrinii R.F.Almeida, Phytotaxa 284(1): 14. 2016. Holotype: BRAZIL. Bahia: Mun. Feira de Santana, Fazenda Cruzeiro do Mocó, 3-XI-2015, fl. fr., R.F. Almeida, A.C. Marques \& M.O.O.Pellegrini 614(HUEFS!; isotypes: CEPEC!, MICH!, NY!, P!, RB!, US!).

Figures 28, 30
Woody vines to scandent shrubs; branches striated, sparsely lenticellate, lenticels brown, sparsely sericeous-velutine to glabrous at age; stipules 0.5-1 mm long, triangular, interpetiolar on branches, epipetiolar on inflorescences, persistent to deciduous. Leaves decussate, reduced in inflorescences; petioles 5-6 mm long, canaliculate, sparsely sericeous-velutine to glabrous at age, eglandular at apex; leaf blades 6-11.3 × 3-6 cm, plane, bullate, elliptic, wide-elliptic to ovate, base cordate to rounded, margin plane, apex acute to acuminate, both sides glabrous, eglandular to 1-2 pairs of glands, $0.4-0.5 \mathrm{~mm}$ diam., up to $3.5-8 \mathrm{~mm}$ from the margins; midvein adaxially impressed, abaxially prominent, 4-6 pairs of secondary veins, arching $45-50^{\circ}$, subopposite to alternate, adaxially impressed, abaxially prominent, reticulum prominent on both sides. Thyrsi (pseudoracemes) or panicles, axillary; main axis 10-28 cm long, cylindrical, striated, sericeous-velutine; cincinni 26-56, 1-flowered, decussate; reduced leaves elliptic, apex acute; bracts 3-3.5 $\times 2-2.25 \mathrm{~mm}$, ovate, plane, sessile, parallel to the peduncle, eglandular to 1-3 pairs of glands at base to middle, both sides sericeous-velutine; peduncle $2.5-3 \times 0.5-0.6 \mathrm{~mm}$, cylindrical, sericeousvelutine; bracteoles 2.5-2.6 × 1.5-1.7 mm, elliptic, concave, sessile, inserted right below the apex of the peduncles, opposite, parallel to pedicel, eglandular to 1 pair of basal glands, both sides sericeousvelutine. Flowers $8-10 \mathrm{~mm}$ diam. at anthesis; floral buds $3-3.5 \times 2.5-3 \mathrm{~mm}$ at anthesis; pedicels 3.8-4 × 0.5-0.6 mm, cylindrical, sericeous-velutine. Sepals 1.5-2.25 × 1-1.25 mm, triangular, appressed to the androecium, apex obtuse to rounded, straight at anthesis, both sides sericeous-velutine; glands yellow turning orange to ocher at age, 1.65-1.75 $\times 0.8-1 \mathrm{~mm}$. Petals yellow turning light orange at age, margin sinuate, anterior-lateral petals not overlapping at anthesis; lateral petals patent at anthesis, limb 3-4 $\times$ 2-2.5 mm, elliptic to obovate, base truncate, adaxially sericeous-velutine distally, abaxially sericeous-velutine; claws 0.75-1 × 0.4-0.5 mm, plane, adaxially glabrous, abaxially sericeous-velutine; posterior petal erect at anthesis, limb 2.8-3 × 1.8-2 mm, elliptic, base truncate, eglandular, adaxially sericeousvelutine distally, abaxially sericeous-velutine; claw $1.8-2 \times 0.62-0.75 \mathrm{~mm}$, plane, adaxially glabrous, abaxially sericeous-velutine. Stamens opposite petals longer than those opposite sepals; filaments $1.25-1.5 \times 0.3-0.4 \mathrm{~mm}$, connate $0.25-0.3 \mathrm{~mm}$ long at base, glabrous; anthers dimorphic, straight, with a 

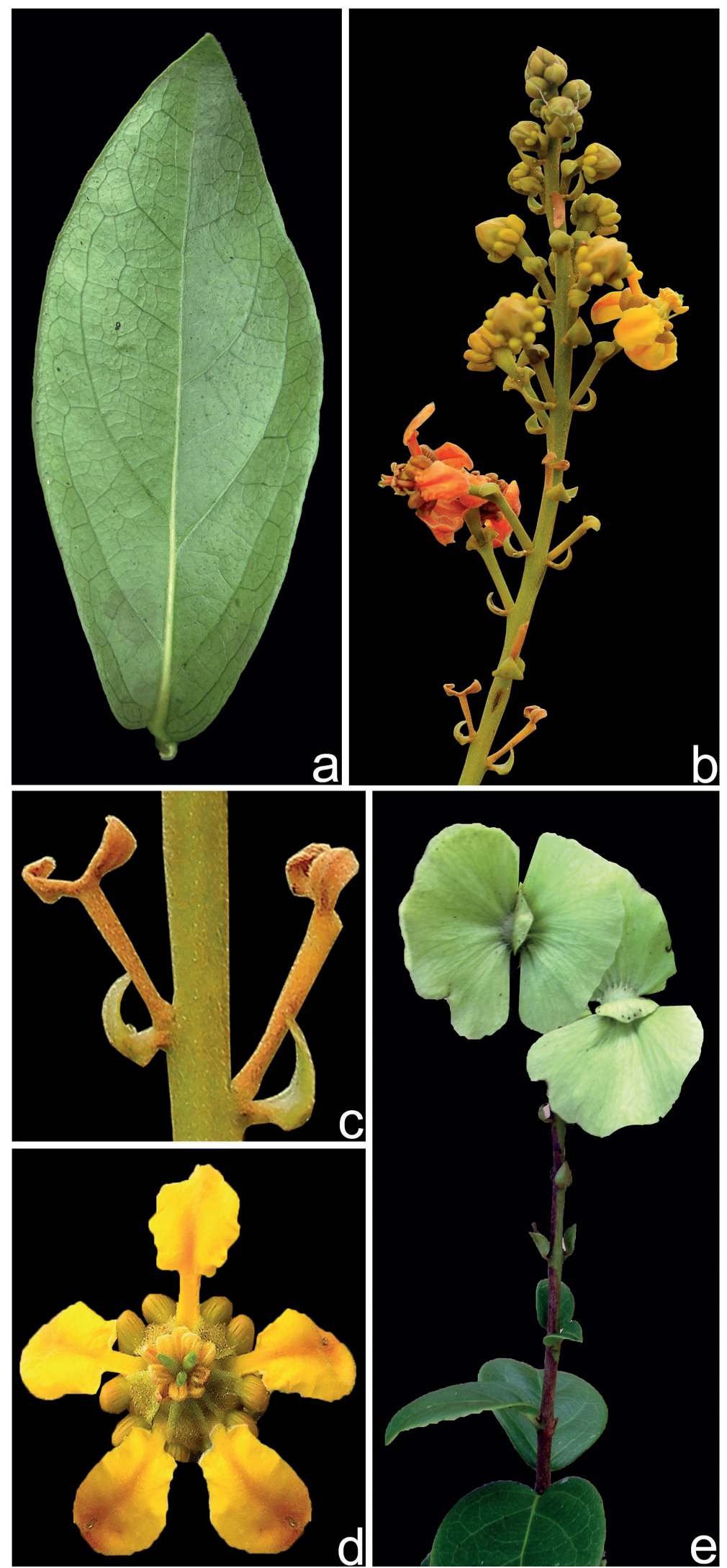

Figure 28. Amorimia pellegrinii R.F. Almeida: a. leaf in abaxial view. b. inflorescence and flowers. c. bract and bracteoles. d. flower in frontal view. e. fruiting branch (photographs by R.F. Almeida). 
glandular connective, $0.8-1 \times 0.4-0.6 \mathrm{~mm}$, reflexed at anthesis, base, connectives and apex pubescent. Ovary 1.4-1.5 × 1.4-1.5 mm, each carpel with primordial lateral and dorsal wings, sericeous-velutine; styles 3 , cylindrical at base and apex, parallel at base, divergent at middle, apex truncate with rounded angle, sericeous-velutine at base, glabrous at middle and apex, anterior style 1.4-1.5 mm long, posterior styles 1.6-1.8 mm long; stigma lateral, crateriform. Samaras green to ocher in vivo; dorsal wing 7-8 $\times$ 2.5-3 mm, very shallowly triangular, margin erose, sinuate, both sides sericeous-velutine; lateral wings 1.2-1.5 $\times 1.2-1.4 \mathrm{~cm}$, flabelliform, margin erose, sinuate, upper angle $40^{\circ}$, lower angle $60^{\circ}$ from the nut, both sides sericeous-velutine; nut 7-8 $\times 4.5-5 \mathrm{~mm}$, ovoid, sericeous-velutine; areole 5-6 $\times 2-3 \mathrm{~mm}$, ovate. Seeds 4-4.5 $\times 2-2.5 \mathrm{~mm}$, testa smooth.

Specimens analyzed: BRAZIL. BAHIA: Mun. Baixa Grande, Fazenda Taquiri, 12-V-1985, fl., Gatto 1 (BAH); Mun. Cachoeira, Barragem de Bananeiras, vale dos rios Paraguaçu e Jacuípe, $12^{\circ} 32^{\prime} \mathrm{S}, 39^{\circ} 05^{\prime} \mathrm{W}$, 120 m, 25-XI-2003, fl., Amorim 3801 (ALCB, CEPEC, HUEFS); loc. cit., 22-III-1956, fl., Lordelo 56-307 (ALCB); loc. cit., Pedra do Cavalo, Barragem de Bananeiras, V-1980, fr., Pedra do Cavalo 8 (CEPEC, HUEFS); loc. cit., XII-1980, fl. fr., Pedra do Cavalo 1021, 1031 (ALCB, CEPEC, HUEFS, HRB, RB); loc. cit., IV-1936, fl., Torrend s.n. (ALCB1928, HUEFS204347, SP); Mun. Castro Alves, 28-III-1994, fl., Carvalho 29 (HUEFS); Mun. Conceição do Coité, Serra do Mucambo, $11^{\circ} 30^{\prime} \mathrm{S}, 39^{\circ} 11^{\prime} \mathrm{W}$, 26-VI-2013, fl. fr., Carvalho 296 (HUEFS); Mun. Feira de Santana, Fazenda Chapada, $12^{\circ} 15 ' 21$ 's, 3905'00”'W, 2-VI-2007, fl., Melo 4790 (HUEFS); loc. cit., distrito de São José, 4-VI-2005, fr., Cruz 32 (HUEFS); loc. cit., Fazenda Cruzeiro do Mocó, $12^{\circ} 13^{\prime} 12^{\prime}$ 'S, 39॰02'44'”, 185 m, 28-V-2013, fl. fr., Queiroz 54 (HUEFS); loc. cit., estrada para Fazenda Cruzeiro do Mocó, em frente ao loteamento Minha Casa Minha Vida, XI-2015, fl. fr., Marques 15, 16 (BHCB); loc. cit., Ipuaçu, inselberg Monte Alto, $12^{\circ} 15^{\prime} 17^{\prime}$ 'S, 39॰04'59”'W, 130 m, 22-VI-2009, fl., Melo 6370 (HUEFS); loc. cit., distrito de Ipuaçu, 12॰13'58'S, 3804'28' W, 7-IV-2005, fl., Couto 35 (CEPEC, HUEFS); loc. cit., 22-VI-2009, fr., Melo 6372 (HUEFS); loc. cit., distrito de Bonfim de Feira, entrada no km 8, Inselberg Monte Alto, 2-V-2008, fl., Oliveira 1553, 1554 (HUEFS); Mun. Iaçu, estrada Iaçu-Milagres, Fazenda Santo Antônio, $12^{\circ} 48^{\prime} 01$ 's, $40^{\circ} 05^{\prime} 44^{\prime}$ 'W, 300 m, 20-VII-2001, fr., Souza 26565

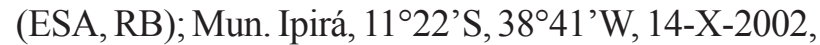
fr., Moura s.n. (HUEFS69135, HUEFS69137); loc. cit., 11'59'49',S, 4005'50'”W, 868 m, 10-IV-2001, fl., Nunes 266 (CEPEC, HUEFS); loc. cit., Fazenda Nova Fanela, ca. $2,5 \mathrm{~km} \mathrm{~S}$ de Ipirá, $12^{\circ} 10^{\prime} 45^{\prime}$ 'S, 3946'12'”W, s.d., fl. fr., Queiroz 10607 (CEPEC, HUEFS); Mun. Iraquara, along road $12 \mathrm{~km}$ from city to BR-242, 600m, 14-VI-1981, fr., Mori 14435 (CEPEC, NY); Mun. Itiuba, a $10 \mathrm{~km}$ de Itiuba, 10³9'28' S, 39॰44'16"'W, 375 m, 28-I-2002, fr., Nascimento 38 (CEPEC, HUEFS); loc. cit., ca. $5 \mathrm{~km}$ em direção a Coité, Fazenda Grotão, 10-V-2002, fl., Nascimento 83 (CEPEC, HUEFS); loc. cit., Serra de Itiúba, vila de Adro, 27-V-1983, fl. fr., Bautista 780 (HRB, MBM); Mun. Jacobina, margem esquerda do rio Jacuípe, 28-III-1985, fl. fr., Bastos 420 (BAH, NY); Mun. Jaguari, caminho do Engenho, estrada para Grotas, $10^{\circ} 08^{\prime}$ 'S, 40 $40^{\circ}$ 'W, 24-VI-2005, fr., Souza-Silva 15, 20 (CEPEC, HUEFS); Mun. Jaíba, periferia do povoado São Roque, $12^{\circ} 15^{\prime} 05^{\prime}$ 'S, 38 $38^{\circ}$ '36”W, 174 m, 22-V-2010, Silva 85 (HUEFS); Mun. Jeremoabo, ca. $12 \mathrm{~km}$ da cidade, 22-V-1978, fl. fr., Souza-Silva 612 (HUEFS, SP); Mun. Mairí, margem direita do rio Jacuípe, 29-III-1985, fl., Oliveira 658 (BAH, CEPEC, HUEFS, NY); Mun. Miguel Calmon, arredores da cidade, 16-VI-1985, fr., Noblick 3876 (CEPEC, HUEFS); loc. cit., estrada Miguel Calmon para Cabeceiras, a $16 \mathrm{~km}$ de Miguel Calmon, $11^{\circ} 21^{\prime} 33^{\prime}$ 'S, 40³3'52”W, 6-IV-2001, fl., Jesus 1299 (CEPEC, HRB, HUEFS); loc. cit., Piemonte da Diamantina, entorno do Parque Sete Passagens, 22-XII-2006, fl. fr., Guedes 13101 (ALCB, MBM); Mun. Milagres, BR116, embaixo da ponte, $13^{\circ} 07^{\prime} 28^{\prime} \mathrm{S}, 38^{\circ} 58^{\prime} 07^{\prime \prime} \mathrm{W}$, 19-VIII-2015, fr., Aona 4208 (HURB); Mun. Monte Santo, 11-I-2006, fr., Guedes 12067 (ALCB); Mun. Piritiba, 31-V-1980, fr., Noblick 1839 (HUEFS); Mun. Pintadas, Fazenda Laguinha, V-2004, fl., Nunes s.n. (CEPEC, RB571); Mun. Quijilingue, lagoa de dentro, a ca. $3 \mathrm{~km}$ E do povoado Quixaba do Mandacaru, $10^{\circ} 55^{\prime}$ S, 3903'W, 270 m, 15-V-2005, fl. fr., Cardoso 518 (HUEFS, NY); Mun. Retirolândia, ca. $5 \mathrm{~km}$ N da cidade, 11²8'45"S, 39²6’32”'W, 250 m, 1-XI-1999, fl., Oliveira 299 (CEPEC, HUEFS); Mun. Riachão

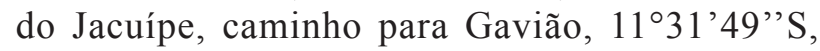
39॰42'30'W, 647 m, 26-III-2000, Guedes 7291 (ALCB, CEPEC, HRB, HUEFS); Mun. Ruy Barbosa, Serra do Orobó, Fazenda Bom Jardim, 12²0'33' S, 40²8'40'”W, 426 m, 25-V-2005, fr., Queiroz 10655 (CEPEC, HUEFS); loc. cit., margem do rio Água Branca, 1204'21''S, 40³3’38”'W, 19-V-2007, fl. fr., 
Santana 542 (ALCB); Mun. Santo Amaro, Oliveira dos Campinhos, 4-IV-1990, fl., Neves 89 (BAH); Mun. Santo Estevão, 10-IV-1990, fl., Batatinha 1 (BAH); Mun. Senhor do Bonfim, Serra da Maravilha, 10²4'16”'S, 40¹2'36”'W, 739 m, 28-VII-2005, fr., Castro 1281 (HUEFS); loc. cit., Serra de Santana, 28-VII-2005, fr., Nunes 1218 (CEPEC, HUEFS); loc. cit., 13-V-1974, fr., Andrade-Lima 7664 (HUEFS, IPA); Mun. Serra Preta, $6 \mathrm{~km}$ do ponto de Serra Preta, Fazenda Santa Clara, 17-VII-1985, fr., Noblick 4228 (CEPEC, HRB, HUEFS); Mun. Tanquinho, estrada para Exu, 12 ${ }^{\circ} 42^{\prime} \mathrm{S}, 39^{\circ} 43^{\prime} \mathrm{W}, 2-\mathrm{VI}-2005$, fl. fr., Carvalho 111, 114 (CEPEC, HUEFS, NY); loc. cit., Fazenda Beira do Rio, 27-V-1999, fl., Vanilda 146 (HUEFS); Tucano, povoado Bizamum, Serra Grande, $10^{\circ} 51^{\prime} 18^{\prime \prime S}, 38^{\circ} 02$ '14"'W, 452 m, 21-IV-2005, fl., Cardoso 493 (HUEFS, NY); loc. cit., 1053'43's, $38^{\circ} 58^{\prime} 47^{\prime}$ 'W, 236 m, 10-IV-2004, fr., Cardoso 40 (HUEFS). Ceará: Mun. Brejo Santo, lote 5, povoado Oitis, reservatório porcos, $07^{\circ} 36^{\prime} 45^{\prime}$ 'S, 38 $38^{\circ}$ ' $17^{\prime}$ 'W, 685 m, 5-VIII-2011, fr., Ferreira 339 (HUEFS, HVASF). Pernambuco: Mun. Bom Conselho, 7-II-1969, fl., Döbereiner \& Tokarnia 498 (PAMG); Mun. Floresta, Floresta do Navio, 24-V-1978, fl. fr., Souza-Silva 650 (HUEFS, SP); Mun. Inajá, Reserva Biológica Serra Negra, 08 39'00”S, 38 23'00”W, 799 m, 3-IX-2009, fr., Carvalho-Sobrinho 2353 (CEPEC, HUEFS, HVASF); Mun. Ipubi, margem da estrada para a mineradora Bonito, 15-IV-2010, fl., Miranda 6171 (FUEL, HUEFS, HST, HSTA, SP); loc. cit., Mina Belo/Lucena, 15-V-2010, fl., Silva s.n. (HST17938); Mun. Santa Maria da Boa Vista, próximo a ponte do rio das Garças, em direção a lagoa, 2-V-1971, fl., Heringer 462 (HUEFS, IPA, R, RB); Mun. São José do Belmonte, Mirandiba, 15-V-1971, fr., Academia Brasileira de Ciências 780 (HUEFS, IPA). Sergipe: Mun. Macambira, Cachoeira de Macambira, 5-III-2013, fl., Matos 84 (ASE); Mun. Riachão do Dantas, 8-IV-1986, fl., Viana 1397 (ASE, IPA); loc. cit., Fazenda Dr. Belmiro, 28-XI-2013, fr., Matos 325 (ASE).

Distribution, habitat and phenology: Amorimia pellegrinii in known to SDTF from northern Bahia State, and surroundings of Ceará, Pernambuco and Sergipe States, Brazil (figure 19). Flowering and fruiting throughout the year.

Conservation status: despite possessing a wide EOO of $118,474.482 \mathrm{~km}^{2}$, A. pellegrinii should be regarded as Endangered [EN, B1ab(iii,iv,v); C2a(i,ii); D2] due to its restricted $\mathrm{AOO}$ (ca. $44.000 \mathrm{~km}^{2}$ ) in anthropically modified Caatinga vegetation.
Etymology: the epithet pays honor to Marco Octávio de Oliveira Pellegrini (b.1990), a Brazilian botanist contributor to the study of the Malpighiaceae from the Atlantic Forest, husband of the author, and additional collector of the type specimen.

Taxonomy notes: Amorimia pellegrinii is similar to $A$. rigida and $A$. velutina due to its plane, bullate, sericeous-velutine leaf blades, with a gland pair near margin or near base, parallel bracteoles, sepals appressed to the androecium with straight apex, petals turning orange at post-anthesis, claws adaxially glabrous, posterior petal eglandular, filaments glabrous, and styles truncate at apex. Nonetheless, A. pellegrinii can be differentiated by its elliptic leaf blades, with 4-6 pairs of secondary veins, peduncles exceeding the bracts at anthesis, and posterior petal claw 0.62-0.75 mm wide.

\subsection{Amorimia rigida (A.Juss.) W.R.Anderson,} Novon 16(2): 183. 2006. Basionym: Hiraea rigida A.Juss., Fl. Bras. Merid. (quarto ed.) 3(21): 14. 1832 [1833]. 三 Mascagnia rigida (A.Juss.) Griseb., Fl. Bras. 12(1): 92. 1858. ETriopterys brachypteris Mohl ex Nied., Pflanzenr. IV, 141: 108 1928. nom. illeg. Lectotype (designated by Anderson 2006): BRAZIL. Minas Gerais: Mun. São Miguel do Jequitinhonha, 1816-1821, fl. fr., A. St.-Hilaire Catal. B1 no. 1501 (P barcode 02429227!; isolectotypes: $\mathrm{P}$ barcode 02429228!, P barcode 02429229!).

Figures 29-30

Woody vines to scandent shrubs; branches striated, sparsely lenticellate, lenticels brown, sparsely sericeous-velutine to glabrous at age; stipules $0.8-1 \mathrm{~mm}$ long, triangular, glabrous, interpetiolar on branches, epipetiolar on inflorescences, persistent to deciduous. Leaves decussate, reduced in inflorescences; petioles 4-5 mm long, canaliculate, sparsely sericeous-velutine to glabrous at age, eglandular at apex; leaf blades 7.7-11.5 $\times 4.2-6 \mathrm{~cm}$, plane, bullate, ovate to elliptic to slightly obovate, base rounded to cuneate, margin plane, apex acute to acuminate, both sides glabrous, eglandular to 2-pairs of glands, $0.4-0.5 \mathrm{~mm}$ diam., near base or up to $6.5-8.5 \mathrm{~mm}$ from margins; midvein adaxially impressed, abaxially prominent, 7-8 pairs of secondary veins, arching $50^{\circ}$, subopposite to alternate, adaxially impressed, abaxially prominent, reticulum prominent on both sides. Thyrsi (pseudoracemes) or panicles, axillary to terminal; main axis $12-15 \mathrm{~cm}$ long, cylindrical, striated, green in vivo, sericeousvelutine; cincinni 26-30, 1-flowered, spirally 
alternate; reduced leaves elliptic, apex acute; bracts 3.2-3.5 $\times 2.1-2.5 \mathrm{~mm}$, ovate, plane, sessile, parallel to peduncle, 1-3 pairs of marginal glands, at base to middle, both sides sericeous-velutine; peduncle 2.5-3 × 0.9-1 mm, cylindrical, sericeous-velutine; bracteoles 2-2.5 × 1.25-1.5 mm, elliptic, concave, sessile, inserted at the apex of the peduncles, parallel to pedicel, eglandular, both sides sericeousvelutine. Flowers $10-12 \mathrm{~mm}$ diam. at anthesis; floral buds 4.5-6 $\times$ 4.5-5 $\mathrm{mm}$ at anthesis; pedicels 5-6.5 × 0.9-1 mm, cylindrical, sericeous-velutine.
Sepals $1.5-2 \times 1-1.5 \mathrm{~mm}$, ovate, appressed to the androecium, apex obtuse to rounded, straight at anthesis, both sides sericeous-velutine; glands yellow turning orange to ocher at age, $2-2.5 \times 0.8-1 \mathrm{~mm}$. Petals yellow turning orange at age, margin sinuate, anterior-lateral petals not overlapping at anthesis; lateral petals patent at anthesis, limb 3-4 × 2-2.5 mm, elliptic, base truncate to hastate, adaxially sericeousvelutine distally, abaxially sericeous-velutine; claws 0.9-1.2 × 0.3-0.4 mm, plane, adaxially glabrous, abaxially sericeous-velutine; posterior petal erect
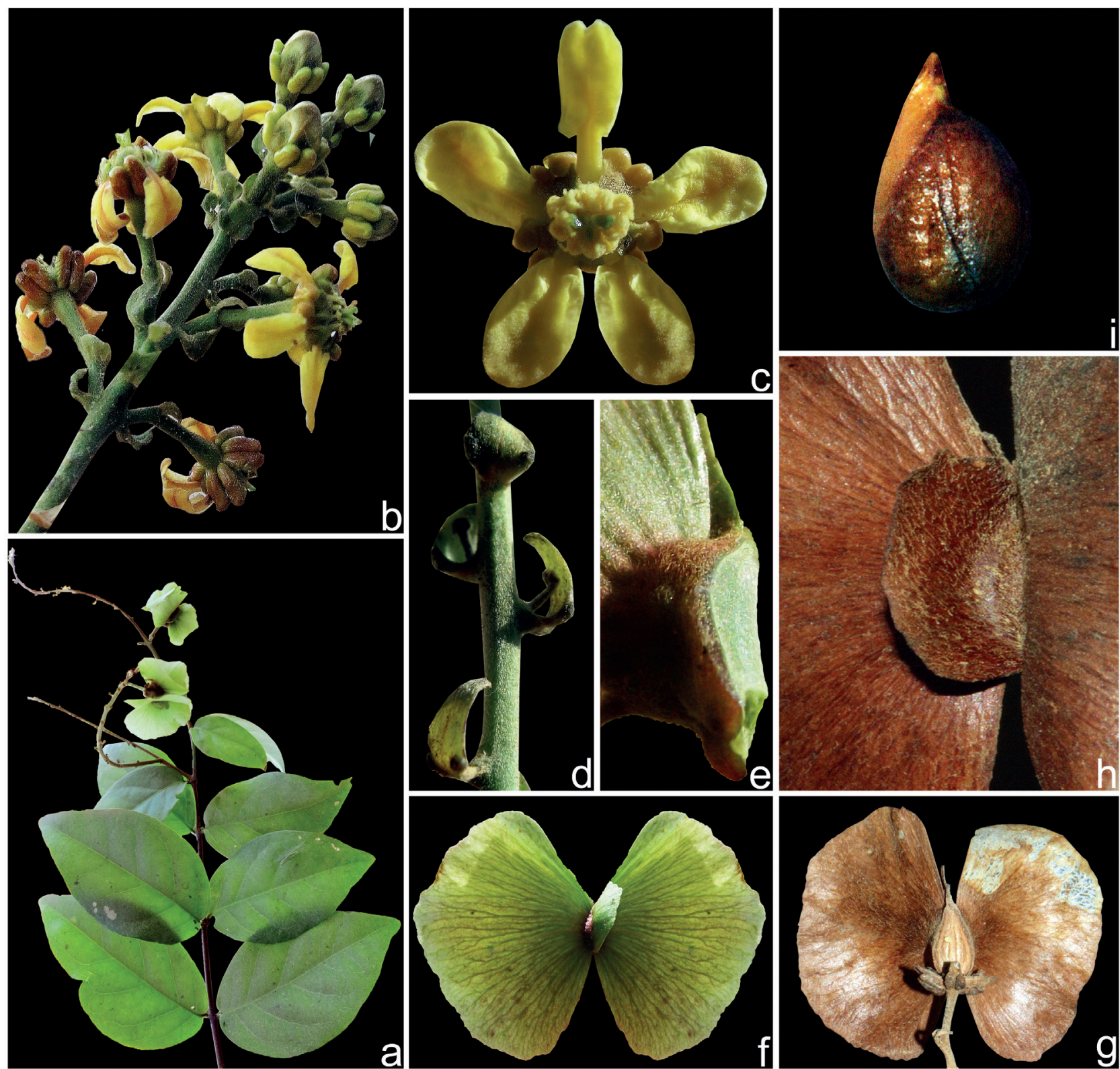

e
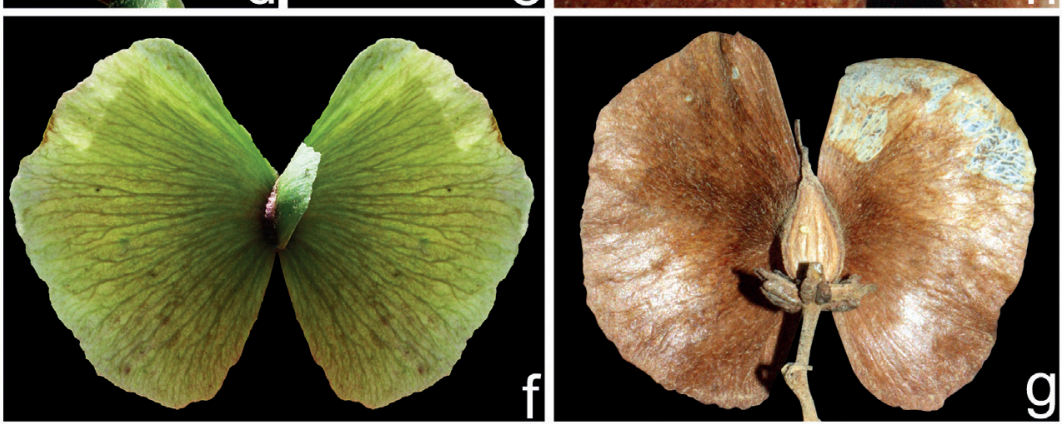

Figure 29. Amorimia rigida (A. Juss.) W.R. Anderson: a. fruting branch. b. inflorescence and flowers. c. flower in frontal view. d. floral bracts. e. areole in side view. f. samara in adaxial view. g. samara in abaxial view showing the areole. h. detail of the dorsal wing. i. seed (photographs a-f by R.F. Almeida, g-i by M.O.O. Pellegrini). 

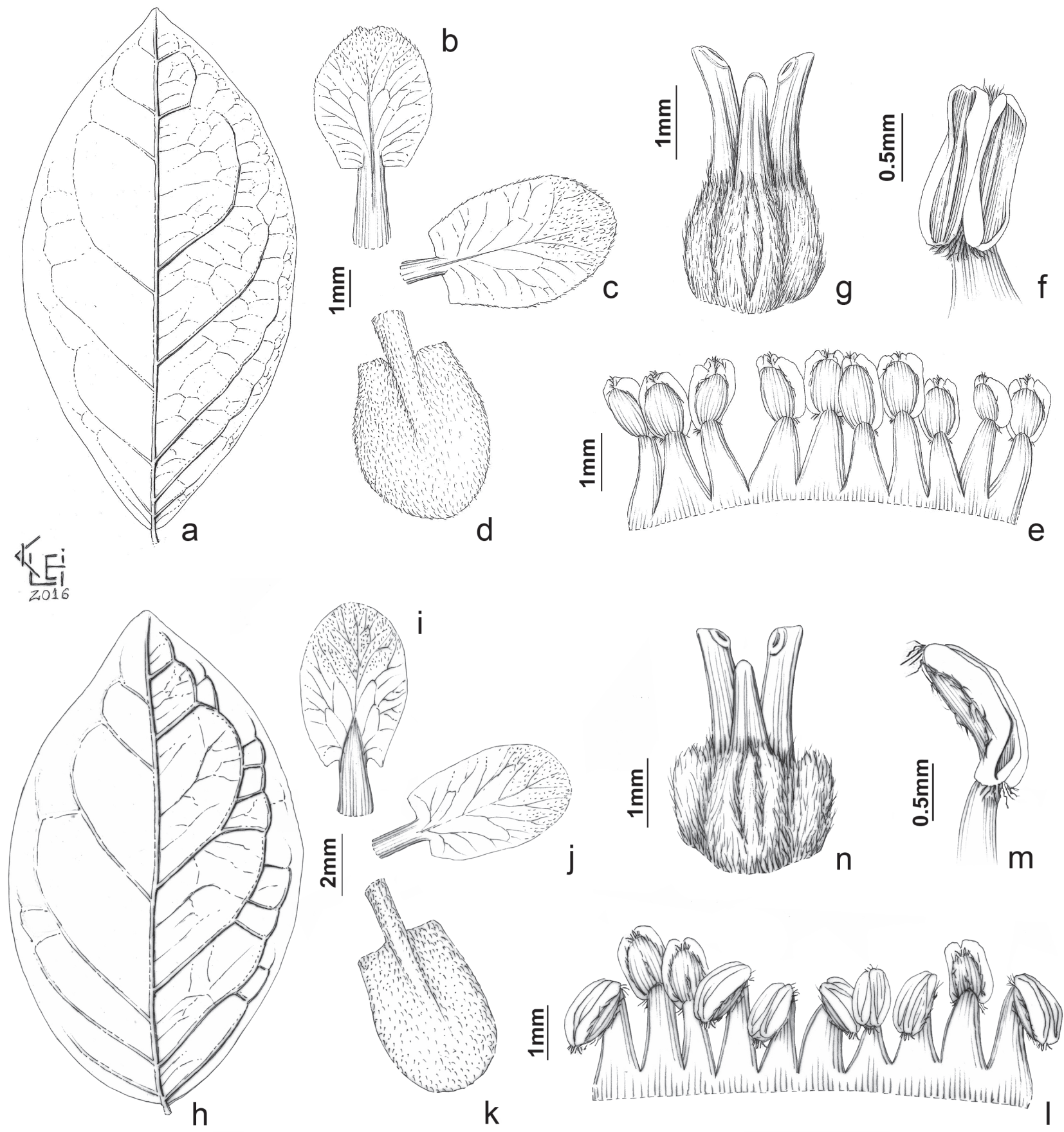

$\mathrm{m}$

Figure 30. Amorimia pellegrinii R.F. Almeida: a. abaxial side of a leaf evidencing vein pattern. b. adaxial side of a posterior petal. c. adaxial side of a posterio-lateral petal. d. abaxial side of a anterio-lateral petal. e. androecium. f. detail of a stamen evidencing anther hairs. g. gynoecium (drawings by Klei Sousa). Amorimia rigida (A. Juss.) W.R. Anderson: h. abaxial side of a leaf evidencing vein pattern. i. adaxial side of a posterior petal. j. adaxial side of a posterio-lateral petal. k. abaxial side of an anterio-lateral petal. 1. androecium. m. detail of a stamen evidencing anther hairs. n. gynoecium (drawings by Klei Sousa). 
at anthesis, limb 3-3.5 $\times 2.8-3 \mathrm{~mm}$, elliptic, base truncate to hastate, eglandular, adaxially sericeousvelutine distally, abaxially sericeous-velutine; claw $0.9-1 \times 0.4-0.5 \mathrm{~mm}$, plane, adaxially glabrous, abaxially sericeous-velutine. Stamens opposite the anterior sepal, lateral-posterior sepals and posterior petal longer than those opposite the lateral petals; filaments $1.25-1.5 \times 0.25-0.4 \mathrm{~mm}$, connate $0.4-0.5 \mathrm{~mm}$ long at base, glabrous; anthers monomorphic, straight, with a glandular connective, $1-1.25 \times 0.4-0.5 \mathrm{~mm}$, reflexed at anthesis, base, connective and apex pubescent. Ovary 1.1-1.25 × 1.4-1.5 mm, each carpel with primordial dorsal and lateral wings, sericeous-velutine; styles 3 , cylindrical at base and apex, parallel at base, divergent at middle, apex truncate with rounded angle, sericeous-velutine at base, glabrous at middle and apex, anterior style 0.9-1 mm long, posterior styles 1.2-1.25 mm long; stigma lateral, crateriform. Samaras green turning pale ocher in vivo; dorsal wing $9-10 \times 4.5-5.5 \mathrm{~mm}$, very shallowly triangular, margin entire, sinuate, both sides sericeous-velutine; lateral wings $1.3-1.5 \times 2.5-2.6 \mathrm{~cm}$, flabelliform, margin erose, sinuate, upper angle $60^{\circ}$, lower angle $75^{\circ}$ from the nut, both sides sericeous-velutine; nut 7-8 $\times 2.5-3 \mathrm{~mm}$, ovoid, sericeous-velutine; areole 6-6.5 $\times 2.5-3 \mathrm{~mm}$, elliptic. Seeds 4.7-5 $\times 3-3.2 \mathrm{~mm}$, testa smooth.

Specimens analyzed: BRAZIL. BAHIA: Mun. Almadina, road Almadina to Ibitupã, ca. $20 \mathrm{~km}$, Fazenda São Roque, ca. $10 \mathrm{~km}$ da entrada do ramal, $14^{\circ} 38^{\prime} 27^{\prime}$ 'S, 3942'47'W, 12-III-2005, fl., Fiaschi 2761 (CEPEC, NY, RB); loc. cit., rodovia para Ibitupan, 12-III-1971, fl., Pinheiro 1132 (CEPEC); Mun. Itagibá, Mata da Botinha, 1410'53"S, 3942'31'"W, 12-VII-2009, fr., Guedes 16327 (ALCB, MBM); Mun. Itajú da Colônia, km 8 da estrara Itajú da Colônia-Pau Brasil, a 3 km do ramal a direita, s.d., fl., Santos 354 (CEPEC); Mun. Itapetinga, 1-VI-1980, fl., Döbereiner 1673 (PAMG); Mun. Itororó, Fazenda Santa Ana, 14.4 $\mathrm{Km}$ of Itajú da Colônia, on road to rio do meio, 1509'01'”S, 3949'57’'W, 200 m, 24-VII-2003, fl., Thomas 13476 (CEPEC, NY); Mun. Jussari, ca. $2.5 \mathrm{~km} \mathrm{~N}$ of Palmira on road to Itajú da Colônia, $15^{\circ} 08^{\prime} 03$ ' S, 39³4'03'”, 300-450 m, 2-II-1999, fl., Thomas 11954 (CEPEC, NY); loc. cit., Fazenda Santa Isabel, rodovia que liga BR 101 a Jussari, 27I-2009, fl., Mattos-Silva 5117 (CEPEC, HUESC); loc. cit., RPPN Serra do Teimoso, entrada 7,5 km da rodovia Jussari/Palmira, Fazenda Teimoso, 1,7 km da entrada, 159'16”'S, 39³1'52”'W, 26-I-2006, fl., Paixão 676 (CEPEC, NY); Mun. Medeiros Neto,
9-IX-1974, fl., Souza s.n. (BAH509); Mun. Posse, near Vitória da Conquista, road to Boa Nova, $\mathrm{km} 5$, 25-I-1973, fl., Gottsberger 24-25173 (BOTU, NY); Mun. Santa Cruz da Vitória, ca. $8.1 \mathrm{~km}$ from the city, fazenda Uruguaiana, 28-VI-2000, fr., Amorim 3565 (CEPEC, NY, SP); loc. cit., Fazenda Boa Fé, 9,3 $\mathrm{km}$ na rodovia para Itajú da Colônia, $15^{\circ} 02$ '24' $\mathrm{S}$, 3947'10'”W, 18-IV-2006, fl., Lopes 655 (CEPEC, NY). Minas Gerais: s. loc., 1838, fl., Claussen s.n. (P4843527); s. loc., 1883, fl., Glaziou 13601 (P); Mun. Araguari, Fazenda da Mata, 23-III-1993, fl., Araújo 838 (HUFU); Mun. Iguatama, Fazenda Faroeste, margem esquerda do rio São Miguel, $20^{\circ} 15^{\prime} 44^{\prime \prime}$, 45 40'15' 'W, 650-700 m, s.dat., fr., Melo 1334 (BHCB); Mun. Jequitinhonha, Reserva Biológica Mata Escura, 27-VII-2013, fl. fr., Almeida et al. 561, 562 (CEPEC, HUEFS, RB); loc. cit., Fazenda Porto Novo, 20-VI-1972, fr., Döbereiner 858 (PAMG, RB); loc. cit., REBIO Mata Escura, XI-2015, fr., Marques 7 (BHCB); Mun. Lagoa Santa, 1907, fl., Warming 838 (P); Mun. Mariana, Serra do Caraça, 19-I-1880, fl., Glaziou 12493 (NY, P); Mun. Matozinhos, Fazenda Cauaua, Mata Cubieri, 5-IV-2004, fl. fr., RodriguesSilva 335 (HUEFS, PMSP); Mun. Prudente de Morais, Fazenda Santa Rita, 25-V-1977, fr., Francisco 11 (PAMG); Mun. Resplendor, parcela para estudos do EIA do Mineroduto Morro do Pilar, 19¹6’37's, 4104'51',W, 23-VI-2013, fl., Saddi 865 (RB); Mun. Rio de Casca, Fazenda Esmeralda, Serra da Mantiqueira, 21-I-1989, fl., Brozek 12 (HUEFS, HRCB); loc. cit., Usina Hidrelétrica de Jurumirim, 3-II-1998, fl. fr., Salino 3953 (BHCB); Mun. Sete Lagoas, CNP-MS, 24-VIII-1982, fl., Cunha 741 (PAMG); Mun. Tumiritinga, 12-IV-1999, fl., Rodrigo s.n. (JPB54379, SP337133, VIC23366); loc. cit., assentamento $1^{\circ}$ de junho, 16-IX-2001, fr., Freitas s.n. (JPB54378, VIC26216).

Distribution, habitat and phenology: Amorimia rigida is known only from Semi-deciduous Forests in the States of Bahia and Minas Gerais, Brazil (figure 19). Flowering from January to July, and fruiting from April to July.

Conservation status: Amorimia rigida possesses a wide EOO (ca. 231,385.245 km²). However, its AOO is considerably smaller (ca. $40,000.00 \mathrm{~km}^{2}$ ), with semi-deciduous forests from State of Minas Gerais being currently reduced to small forest fragments, probably reflecting on the few modern collections of $A$. rigida in this state. The only recent collection from a population protected by a conservation unit is that 
from the type locality of $A$. rigida in Jequitinhonha municipality in Northern Minas Gerais (Reserva Biológica da Mata Escura). Thus, A. rigida should be considered Endangered [EN, B1 ab(iii,iv,v); C2a(i,ii); D2].

Etymology: the epithet probably refers to the rigid texture of its leaf blades.

Taxonomic notes: Amorimia rigida, in its current circumscription, is morphologically similar to $A$. velutina, due to its plane, ovate to wide elliptic, bullate, sericeous-velutine leaf blades, with 7-8 pairs of secondary veins and a pair of glands near base or near margin, peduncles not exceeding the bracts, bracts and bracteoles parallel, sepals appressed to the androecium, with straight apex at anthesis, petal claws adaxially glabrous, petals turning orange at post-anthesis, eglandular posterior petal, with claw 0.4-0.5 mm wide, filaments glabrous, and style with truncate apex. Nonetheless, A. rigida can be differentiated by its elaiophores turning orange to brown at post-anthesis ( $v$ s. red in A. velutina), lateral petals patent at anthesis ( $v s$. reflexed), anterior-lateral petal divergent ( $v s$. overlapping), filaments opposite the anterior sepal, posteriorlateral sepals and posterior petal longer than those opposite lateral petals ( $v S$. opposite sepals shorter than those opposite petals), anthers pubescent at apex, connectives and base ( $v s$. pubescent only at base), and samaras with triangular dorsal wing (vs. obtrapezoidal bearing two triangular projections at apex).

1.8. Amorimia velutina W.R.Anderson, Novon 16(2): 185. 2006. Holotype: BRAZIL. Minas Gerais: Mun. Itinga, Fazenda Timirim, property of Dr. Alexandre,

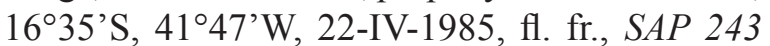

(mounted on two sheets MICH barcode 1003064!). Figures 31-32

Woody vines to scandent shrubs; branches striated, sparsely lenticellate, lenticels brown, sparsely sericeous-velutine to glabrescent at age; stipules 0.7-1.5 mm long, triangular, glabrous, interpetiolar on branches, epipetiolar on inflorescences, persistent to deciduous. Leaves opposite, reduced in inflorescences; petioles 2-4.5 mm long, canaliculate, sericeousvelutine to glabrescent at age, eglandular; leaf blades $5-10 \times 3.5-5 \mathrm{~cm}$, plane, bullate, ovate, elliptic to obovate, base rounded to attenuate, margin plane, apex acute, both sides sericeous-velutine to glabrous at age, eglandular to 1-pair of glands, 0.4-0.5 mm diam., near base or distally up to $2-5 \mathrm{~mm}$ from margins; midvein adaxially impressed, abaxially prominent, secondary veins $7-8$ pairs, arching $40^{\circ}$, subopposite to alternate, adaxially impressed, abaxially prominent, reticulum prominent on both sides. Thyrsi (pseudoracemes) or panicles, axillary or terminal; main axis $4-20 \mathrm{~cm}$ long, cylindrical to slightly flattened, slightly striated, ocher in vivo, sericeous-velutine; cincinni (10-)30-50, 1 -flowered, spirally alternate; reduced leaves wideelliptic, apex mucronate; bracts 3-4.5(-6) × 1.5-3 mm, ovate, plane, elliptic to lanceolate, petiolate, leaf-like, parallel to the peduncle, 2 pairs of glands at base, both sides sericeous-velutine; peduncle 1-4.5 × 0.9-1.1 mm, cylindrical, velutine; bracteoles 2-4 × 1-2 mm, elliptic, concave, sessile, inserted at the apex of peduncles, parallel to the pedicel, 1 pair of glands at base, both sides sericeous-velutine. Flowers 9-11 mm diam. at anthesis; floral buds 4-4.2 × 4.2-4.4 $\mathrm{mm}$ at anthesis; pedicels 3-5.5 $\times 0.9-1.1 \mathrm{~mm}$, cylindrical, sericeousvelutine. Sepals $2.8-3 \times 1.5-1.8 \mathrm{~mm}$, ovate, appressed to the androecium, apex obtuse to rounded, straight to revolute at anthesis, both sides sericeous-velutine; glands yellow turning reddish at age, 2-3.2 $\times 0.9-1 \mathrm{~mm}$. Petals yellow turning dark orange, margins sinuate, anterior-lateral petals overlapping at anthesis; lateral petals reflexed at anthesis, limb 3.5-5 × 2.5-3.5 mm, elliptic, base truncate to hastate, adaxially sericeousvelutine distally, abaxially sericeous-velutine; claws $1-1.5 \times 0.3-0.4 \mathrm{~mm}$, plane, adaxially sericeousvelutine, abaxially sericeous-velutine; posterior petal erect at anthesis, limb 3-4 × 2.2-3.5 mm, elliptic, base truncate at base, eglandular, adaxially sericeousvelutine distally, abaxially sericeous-velutine; claw 2-2.7 $\times 0.5-0.7 \mathrm{~mm}$, plane, adaxially sericeousvelutine, abaxially sericeous-velutine. Stamens opposite petals longer than those opposite sepals; filaments $1.5-2.2 \times 0.25-0.3 \mathrm{~mm}$, connate $0.4-0.5 \mathrm{~mm}$ long at base, glabrous; anthers dimorphic, straight, with a glandular connective, $0.5-1 \times 0.4-0.5 \mathrm{~mm}$, reflexed in anthesis, pubescent at base, glabrous at connective and apex. Ovary 0.6-1.5 × 0.8-1 mm, each carpel with primordial dorsal and lateral wings, sericeous-velutine; styles 3, cylindrical at base and apex, parallel at base, divergent at middle, apex truncate with acuminate angle, sericeous-velutine at base, glabrous at middle and apex, anterior style 0.6-1.3 mm long, curved, posterior styles $0.9-1.7 \mathrm{~mm}$ long, erect; stigma lateral, crateriform. Samaras yellowish in vivo; dorsal wing 7-9 $\times 3.5-5 \mathrm{~mm}$, pentagonal with two triangular projections at apex, margin erose, sinuate, both sides sericeousvelutine; lateral crests between dorsal and lateral 

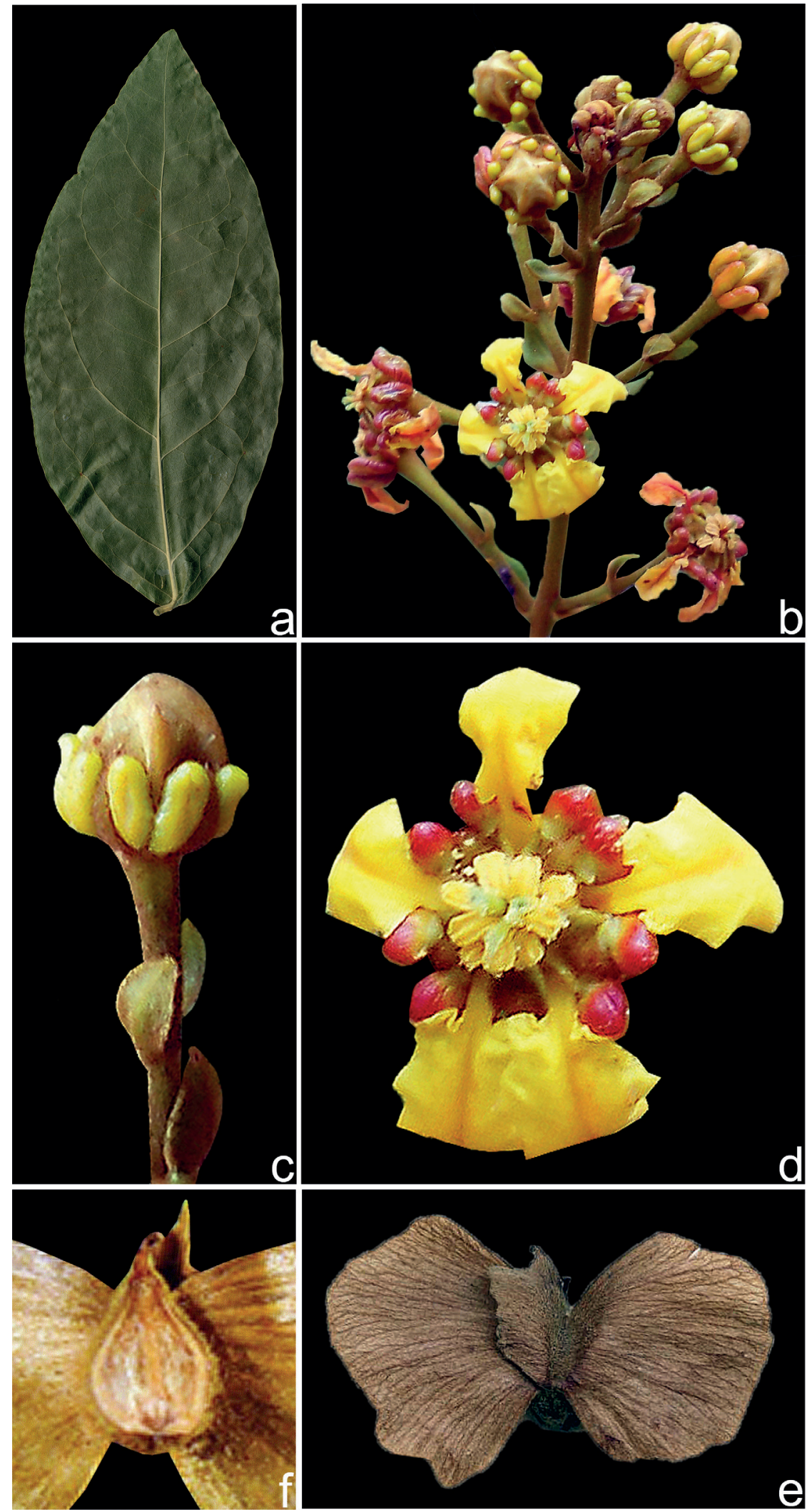

Figure 31. Amorimia velutina W.R. Anderson: a. leaf in abaxial view. b. detail of the inflorescence and flowers. c. floral bud. d. flower in frontal view. e. samara in dorsal view. f. samara in frontal view showing the areole (photographs a-d by L.C. Marinho, e-f by W. R. Anderson). 

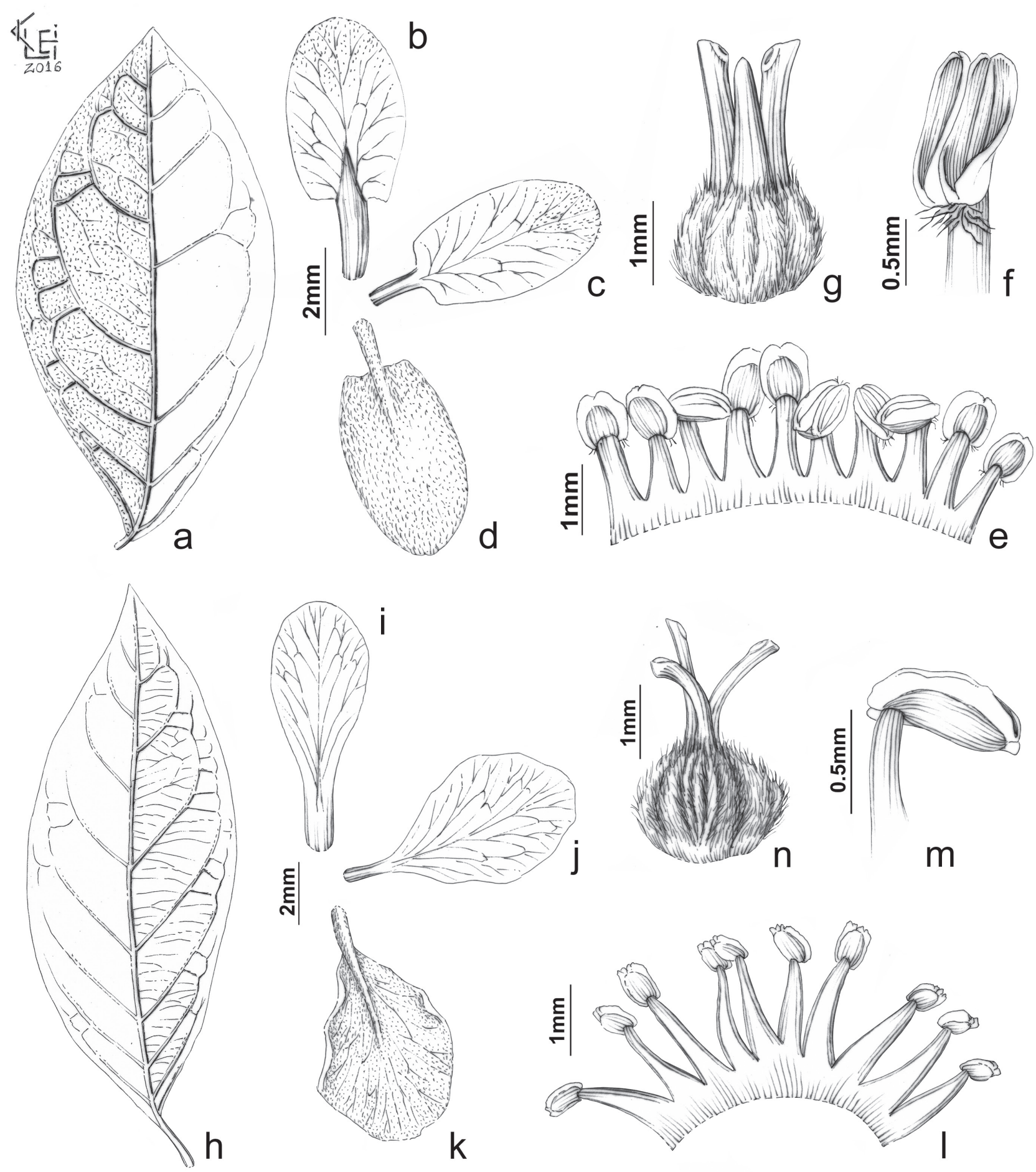

Figure 32. Amorimia velutina W.R. Anderson. a. abaxial side of a leaf evidencing vein pattern. b. adaxial side of a posterior petal. c. adaxial side of a posterio-lateral petal. d. abaxial side of a anterio-lateral petal. e. androecium. f. detail of a stamen evidencing anther hairs. g. gynoecium (drawings by Klei Sousa). Amorimia amazonica (Nied.) W.R. Anderson: h. abaxial side of a leaf evidencing vein pattern. i. adaxial side of a posterior petal. $\mathrm{j}$. adaxial side of a posterio-lateral petal. $\mathrm{k}$. abaxial side of a anterio-lateral petal. 1. androecium. $\mathrm{m}$. detail of a stamen evidencing anther hairs. n. gynoecium (drawings by Klei Sousa). 
wings; lateral wings 10-15 $\times 9-10 \mathrm{~mm}$, flabelliform, margin erose, sinuate, upper angle $30^{\circ}$, lower angle $70^{\circ}$ from the nut, both sides sericeous-velutine; nut 6-7 $\times 2.7-3.1 \mathrm{~mm}$, narrowly ovoid, sericeousvelutine; areole 5.5-6 $\times$ 2-3.5 mm, triangular. Seeds $4.2-4.5 \times 2.5-3 \mathrm{~mm}$, testa smooth.

Specimens analyzed: BRAZIL. BAHIA: Mun. Boa Nova, Parque Nacional de Boa Nova, $14^{\circ} 21^{\prime} \mathrm{S}$, $40^{\circ} 15^{\prime} \mathrm{W}, 600$ m, 25-II-2013, fl., Marinho 396 (CEPEC, HUEFS); Mun. Caetité, Fazenda Baixa Grande, caminho para Pajeú do Vento, $14^{\circ} 04^{\prime} 03^{\prime}$ 'S, 42³8'12”W, 820 m, 9-II-1997, Stannard 5312 (ALCB, CEPEC, HRB, HUEFS, SPF); Mun. Contendas do Sincorá, ca. $26 \mathrm{~km}$ na estrada em direção a Maracás, 9-IV-1999, fl., Amorim 2772 (CEPEC, MBML, MO, NY, SP); loc. cit., FLONA Sincorá, XI-2015, fr., Marques 6 (BHCB); Mun. Coribe, estrada de são Félix do Coribe para Coribe, $22.4 \mathrm{~km}$ vilarejo Colônia,

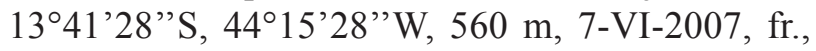
Lopes 1340 (CEPEC, HUEFS); loc. cit., Alagoinha, $14^{\circ} 56^{\prime} 00^{\prime}$ 'S, 4443'56”'W, 10-IV-2005, fr., Castro 1193 (HUEFS); Mun. Iramaia, Fazenda Segredo, 5-XII-2001, fl., Leal 246 (EBDA); Mun. Itaeté, Chapada Diamantina, próximo ao poço encantado, 12 ${ }^{\circ} 98^{\prime} 64^{\prime}$ 'S, 40 97'25"W, 900 m, 13-XI-2014, fr., Guedes 23072 (ALCB); Mun. Jequié, barragem de pedra, pela BR 330 a $16 \mathrm{~km}$ da BR 116, 19-II-2011, fl., Macedo 2112 (HUESB, JPB, NY); Mun. Livramento do Brumado, on road to Brumado, 10-IV-1991, fl., Lewis 1971 (CEPEC, K, NY, SP). Mun. Maracás, Fazenda Tanquinho, ca. 20 km N de Maracás, ramal para Fazenda Santa Rita, na estrada para Planaltino, 30-VI-1993, fl., Queiroz 3259 (CEPEC, HUESC); loc. cit., Águia Branca, 23-VI-2010, fr., Queiroz 4539, 4590, 4701 (HRB); Mun. Rio de Contas, ca. 6 km da cidade em direção a Livramento do Brumado, 800 m, 6-III-1994, fl., Souza 5254 (CEPEC, ESA, SPF); Mun. Tanhuaçu, Floresta Nacional Contendas do Sincorá, trilha do rio Cumbuca, 13-V-2011, fr., Marinho 31 (HUEFS); loc. cit., $50 \mathrm{~m}$ da sede, via rio Cumbuca, 13 ${ }^{\circ} 55^{\prime} 18^{\prime}$ 'S, 41 ${ }^{\circ} 07^{\prime} 08^{\prime}$ 'W, $377 \mathrm{~m}$, 6-II-2015, fl., Aona 3852 (ALCB, HUEFS, HURB); loc. cit., ramal contrário a grade de estrada para sede,

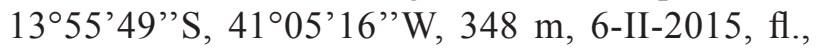
Aona 3892 (ALCB, HUEFS, HURB); Mun. Urandi, Fazenda Feijão Preto, $7 \mathrm{~km}$ de Urandi no sentido Urandi/Guanambi, 1443'59''S, 42³9'00'W, 689 m, 9-IV-2002, fr., Jost 495 (HUEFS, HRB). Goiás: Mun. Formoso, region Southern Serra Dourada, $13^{\circ} 45^{\prime} \mathrm{S}$, 4850’W, 20-VI-1956, fl., Dawson 15006 (P); Mun.
Monte Alegre, Sumidouro, 23-V-2008, fl., Cordeiro 2678 (MBM). Minas Gerais: Mun. Chapada do Norte, Fazenda Mariléo, 21-V-1990, fr., Lima 163 (PAMG); Mun. Itaobim, margem da BR-116, próximo a ponte sobre o Rio Jequitinhonha, 28-VII-1984, fl., Pirani s.n. (HUEFS203426, SPF); Mun. Mato Verde, São João do Bonito, estrada para as lavras na serra geral, $2.5 \mathrm{~km}$ de São João Bonito, $12.5 \mathrm{~km}$ rodovia Mato Verde-Monte Azul, BR 122, 15'18'21'S, 4249'37”W, 7-VI-2004, fl. fr., Pirani 5462 (HUEFS, SPF); Mun. Medina, entre Itaobim e Medina, $16^{\circ} 50^{\prime} 60^{\prime} \mathrm{S}, 41^{\circ} 28^{\prime} 97^{\prime} \mathrm{W}$, 3-IX-2008, fr., Oliveira 1611 (HUEFS); loc. cit., Reservatório do Córrego Ribeirão, 26-V-1999, fr., Salino 4658 (BHCB, SP); Mun. Pedra Azul, ca. 5 km NW da cidade em direção a BR-116, 10-II-1994, fl., Souza 5155 (CEPEC, ESA, SPF); loc. cit., Reservatório do Córrego Soberbo, 28-V-1999, fr., Salino 4713 (BHCB, SP).

Distribution, habitat and phenology: Amorimia velutina is known from SDTF in Southwestern Bahia, Eastern Goiás, and Northern Minas Gerais States, Brazil (figure 19). Flowering and fruiting throughout the year.

Conservation status: Amorimia velutina possesses a wide EOO of ca. 130,780.585 $\mathrm{km}^{2}$. Nonetheless, it possess a restricted AOO (ca. $28.000 \mathrm{~km}^{2}$ ), accentuated by the anthropically modified Caatinga vegetation, conversion of natural habitats into wind farm sites, and deforestation for the implementation of soy crops. Thus, A. velutina should be regarded as Endangered [EN, B1ab(iii,iv,v); C2a(i,ii); D2].

Etymology: the epithet makes reference to the velutine indumenta on its leaf blades.

Taxonomic notes: Amorimia velutina is similar to $A$. andersonii and $A$. rigida due to its plane leaf blades, with a pair of glands near margin or near base, eglandular posterior petal, and posterior petal claw 0.3-0.5 mm wide. Nonetheless, A. velutina can be differentiated by its lateral petals reflexed at anthesis, filaments opposite sepals shorter than those opposite petals, anthers pubescent only at base, and samaras with obtrapezoidal dorsal wing, bearing two triangular projections at apex.

2. Amorimia W.R.Anderson subg. Uncinae R.F.Almeida, Phytotaxa 313(3): 244. 2017. Type: Amorimia pubiflora (A.Juss.) W.R.Anderson.

Differs from A. subg. Amorimia by its stipules narrowly triangular; bracteoles plane or plane 
with revolute margins or concave; sepals adaxially glabrous; petals obovate to spatulate, base cuneate, adaxially glabrous (occasionally distally pubescent in A. septentrionalis), claws plane; anthers usually glabrous (occasionally pubescent in A. pubiflora and $A$. septentrionalis); styles with apex uncinate to occasionally apiculate; pollen grains spherical; samaras with dorsal wing usually continuous to the base of the nut.

Notes: this subgenus includes seven species (i.e., $A$. amazonica, A. camporum, A. concinna, A. kariniana, A. pubiflora, A. septentrionalis, and A. tumida) mostly restricted to SDTF from Northwestern and Northeastern South America (figure 33).

Key to the species of Amorimia subg. Uncinae

\section{Filaments or anthers pubescent}

2. Leaf blade without a pair of glands at base, inflorescences with up to 8 flowers, pedicel thickened in fruits, filaments completely pubescent, style pubescent near apex, samaras metallic green to ocher; Atlantic Forest from northern State of Rio de Janeiro, Brazil

A. tumida

2. Leaf blade with a pair of glands at base, inflorescences with more than 10 flowers, pedicel thin in fruits, filaments completely glabrous, styles glabrous near apex, samaras pale green to ocher; Caatinga, Cerrado or Pantanal domains

3. Stem velutine, sparsely lenticellate; leaf blades cordate or subcordate at base, velutine; petals adaxially glabrous; Cerrado domain in central Brazil and adjacent areas

A. pubiflora

3. Stem tomentose, densely lenticellate; leaf blades cuneate or rounded at base, tomentose; petals adaxially sparsely sericeous-velutine; Caatinga domain in Northeastern Brazil ...... A. septentrionalis

1. Filaments or anthers glabrous

4. Petals only sparsely sericeous abaxially in center of limb, otherwise glabrous; bracts filiform, eglandular; leaf glands marginal; northern Colombia

A. concinna

4. Petals densely and evenly pubescent over the whole abaxial surface; bracts lanceolate, usually biglandular near base; leaf glands borne in abaxial surface, at least the distal glands set in from margin, the glands at base of lamina sometimes almost marginal

5. Branches with blackish lenticels, stipules 0.8-1.1 mm long; petiole 0.4-1.5 mm long, lamina sparsely sericeous below, soon glabrate or sericeous only on midrib with straight appressed hairs; samaras with areoles narrowly triangular to triangular; southwestern Amazonia of Bolivia, Brazil and Peru

A. amazonica

5.Branches with whitish lenticels, stipules 0.3-0.7 mm long; petiole longer than $2 \mathrm{~mm}$ long, lamina at least velutine below, the hairs persistent or the lamina glabrescent at maturity; samaras with areoles narrowly ovate to ovate to elliptic

6. Petiole of larger leaves (5-)7-10 mm long; sepals mostly appressed in anthesis; posterior petal strongly differentiated from lateral petals, with a much longer claw and smaller limb; limb of lateral petals 7.5-8.5 × 6-7 mm; lateral wings of samara 30-42 $\times 20-30 \mathrm{~mm}$; dorsal wing of samara extending to base of nut or nearly so; Ecuador .

6. Petiole of larger leaves up to $6 \mathrm{~mm}$ long, mostly shorter; sepals revolute at apex in anthesis; posterior petal only moderately differentiated from lateral petals, the claw thicker and slightly longer, the limb somewhat smaller; limb of lateral petals 5.5-8 $\times 4-6 \mathrm{~mm}$; lateral wings of samara $14-30 \times 6-18 \mathrm{~mm}$; dorsal wing of samara usually extending at most to middle of nut, from there to base represented only by a rib; Cajamarca and San Martin, Peru

2.1. Amorimia amazonica (Nied.) W.R.Anderson, Novon 16(2): 179. 2006. Basionym: Mascagnia amazonica Nied.,Arbeiten Bot.Inst.Königl.Lyceums Hosianum Braunsberg 8: 59. 1926. Lectotype (designated here): BRAZIL.Amazonas: Seringal São
Francisco, Rio Acre, VIII-1911, fl., E. Ule 9478(MG barcode 014323 !; isotypes: $\mathrm{G}$ barcode 00352819 !, K barcode 000427418 !, L barcode 0064048 !, MO barcode 2155280!, NY barcode 00067646!).

Figures 32, 34 
Woody vines to scandent shrubs; branches striated, sparsely lenticellate, lenticels blackish, sparsely sericeous-velutine to glabrescent at age; stipules $0.8-1.1 \times 0.3-0.5 \mathrm{~mm}$ long, narrowly triangular, sericeous-velutine, interpetiolar on branches, epipetiolar on inflorescences, persistent to deciduous. Leaves opposite, reduced in inflorescences; petioles $0.4-1.5 \mathrm{~mm}$ long, canaliculate, sparsely sericeous-velutine to glabrescent at age, eglandular to 1 pair of glands at apex; leaf blades 5-11.3 × 2.2-6.5 cm, plane, not bullate, narrow-elliptic, elliptic to wideelliptic, base cuneate, margin plane, apex short to long acuminate, both sides sparsely sericeous-velutine at young and glabrescent at age, eglandular to 2-pairs of glands abaxially, 0.3-0.4 mm diam., near base or up to 3-4 mm of margins; midvein adaxially impressed, abaxially prominent, secondary veins 6-7 pairs, arching $50^{\circ}$, subopposite to alternate, adaxially impressed, abaxially prominent, reticulum conspicuous on both sides. Thyrsi (pseudoracemes)

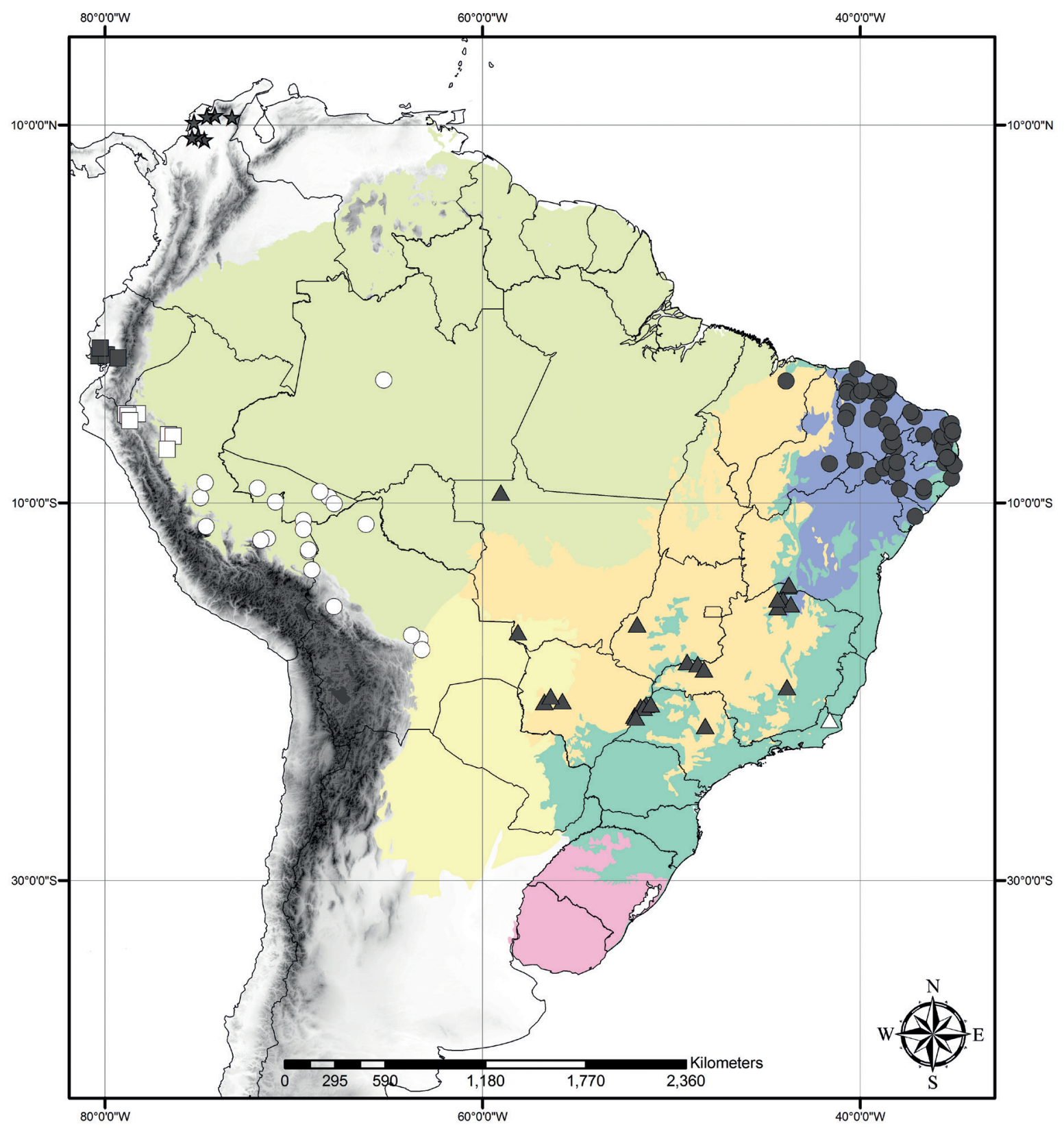

Figure 33. Distribution map of Amorimia subg. Uncinae. - Amorimia septentrionalis. ○ - Amorimia amazonica. $\mathbf{\Delta}$ - Amorimia pubiflora. $\Delta$ - Amorimia tumida. - Amorimia kariniana. $\square$ - Amorimia camporum. Black star - Amorimia concinna. Pink - Pampa, dark green Atlantic Forest, violet - Caatinga, light green - Amazon Forest, and yellow - Chaco/Pantanal. 
or panicles, axillary to terminal; main axis $6-10 \mathrm{~cm}$ long, flattened, irregularly longitudinally costate, sericeous-velutine; cincinni 24-26, 1-flowered, spirally-alternate; reduced leaves widely elliptic, apex mucronate; bracts 3-3.5 $\times 1-1.2 \mathrm{~mm}$, lanceolate, plane, petiolate, parallel to the peduncle, 1 pair of marginal glands at base, both sides sericeous-velutine; peduncle 2-3.5 $\times 0.5-0.6 \mathrm{~mm}$, cylindrical, sericeousvelutine; bracteoles 2.5-3.5 $\times 0.4-0.6 \mathrm{~mm}$, lanceolate, conduplicate, sessile, inserted right below the apex of peduncles, opposite, parallel to the pedicel, eglandular to 2-glandular at margins, both sides sericeousvelutine. Flowers $0.8-1.7 \mathrm{~mm}$ diam. at anthesis; floral buds $0.45-0.6 \times 0.35-0.4 \mathrm{~mm}$ at anthesis; pedicels 5-6 $\times$ 0.5-0.6 mm, cylindrical, sericeous-velutine. Sepals 2.5-3 $\times 1-1.5 \mathrm{~mm}$, triangular, not appressed to the androecium, apex acute, revolute at anthesis, adaxially glabrous, abaxially sericeous-velutine; glands yellow turning ocher at age, 1.5-1.6 × 1-1.1 mm. Petals yellow turning orange on claws at age, margin sinuate, anterior-lateral petals not overlapping; lateral petals patent at anthesis, limb 6.5-7 $\times 4.5-5 \mathrm{~mm}$, wide elliptic to obovate, base cuneate, adaxially glabrous, abaxially sericeous-velutine; claws 1.5-1.6 × 0.5-0.6 mm, plane, adaxially glabrous, abaxially sericeous-velutine; posterior petal erect and slightly curved at anthesis, limb 5-5.1 $\times 4-4.1 \mathrm{~mm}$, obovate, base cuneate, 1-pair of reddish glands at base, adaxially glabrous, abaxially sericeous-velutine; claw 2-2.1 × 1-1.1 mm, plane, adaxially glabrous, abaxially sericeousvelutine. Stamens opposite petals longer than those opposite sepals; filaments $2-2.5 \times 0.34-0.35 \mathrm{~mm}$, connate ca. $0.2 \mathrm{~mm}$ long at base, glabrous; anthers monomorphic, straight, with a glandular connective, 0.9-1.1 $\times$ 0.5-0.6 mm, reflexed at anthesis, glabrous. Ovary 2.4-2.5 × 1.4-1.5 mm, each carpel with primordial dorsal and lateral wings, sericeous-velutine; styles 3, cylindrical at base, laterally flattened at apex,
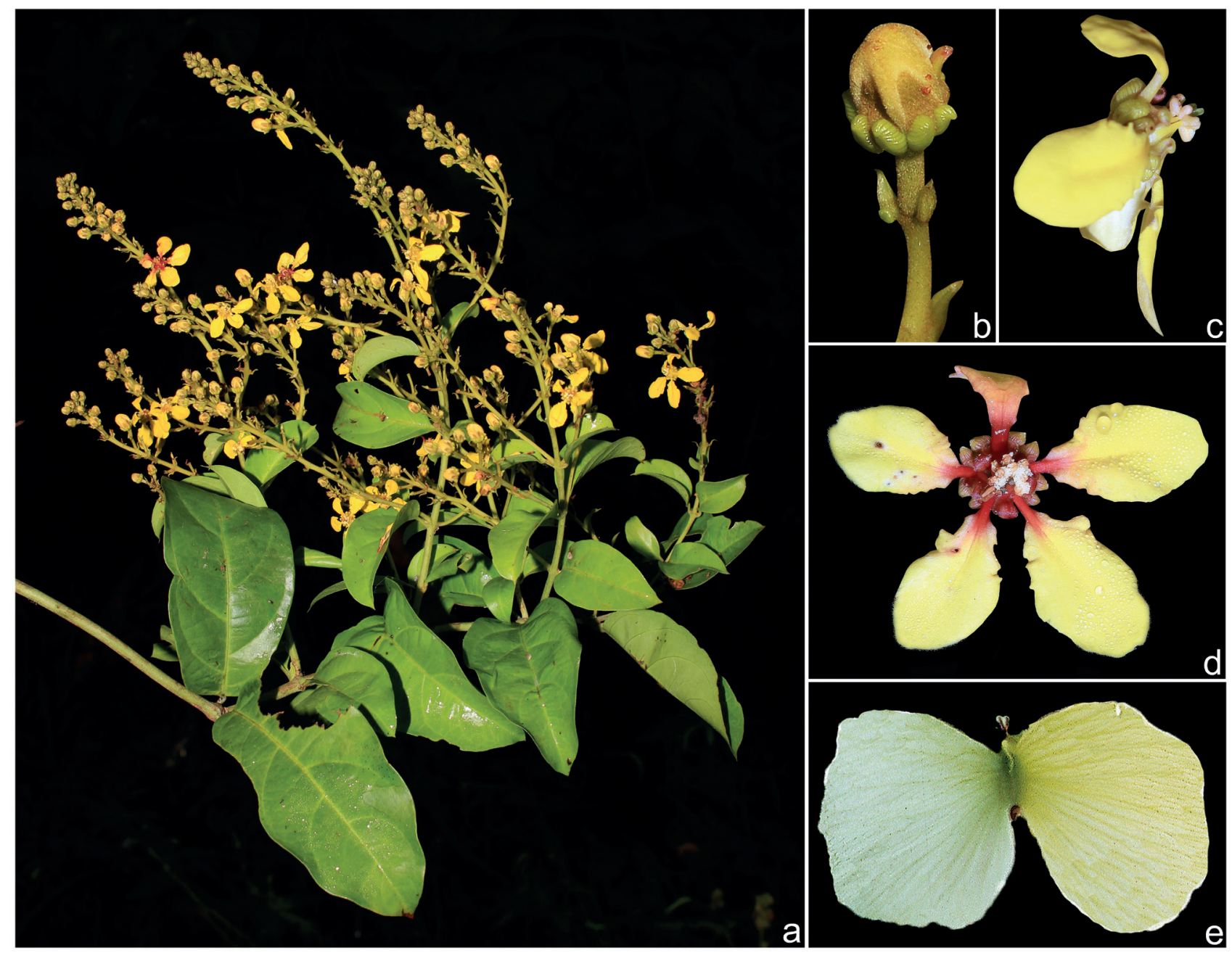

a

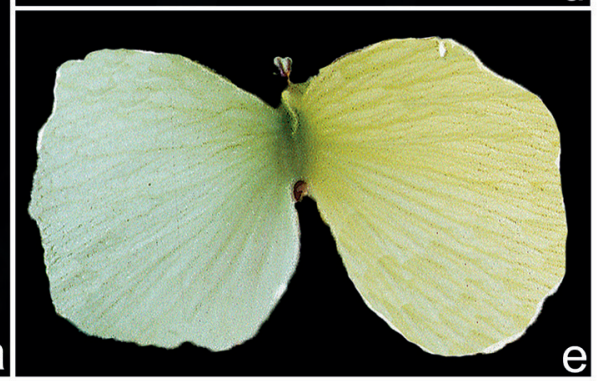

Figure 34. Amorimia amazonica (Nied.) W.R. Anderson: a. habit. b. floral bud in side view. c. flower in side view. d. flower in frontal view. e. samara in dorsal view (photographs a-d by H. Medeiros, e by D. Daly). 
parallel at base, divergent at middle, apex apiculate, sericeous-velutine at base, glabrous at middle and apex, anterior style 1.4-1.45 mm long, posterior styles 1.55-1.6 mm long; stigma lateral, crateriform. Samaras green to yellowish in vivo; dorsal wing reduced to a crest or 6-6.1 $\times 2-2.1 \mathrm{~cm}$, depressed obovate, margin entire to irregularly dentate, plane, both sides sericeous-velutine; lateral wings $2.5-2.6 \times 1.5-1.6 \mathrm{~cm}$, flabelliform, margin erose, sinuate, upper angle $70^{\circ}$, lower angle $80^{\circ}$ from the nut, both sides sericeousvelutine; nut 6-8 $\times 3-4 \mathrm{~mm}$, narrowly ovoid, sericeousvelutine; areole 5.5-5.6 $\times 2-2.1 \mathrm{~mm}$, narrowly triangular to triangular. Seeds $4.5-6 \times 2-3.5 \mathrm{~mm}$, testa smooth.

Specimens analyzed: BOLIVIA. Bens: Mun. Vaca Diez, laguna Tumi Chucua, $30 \mathrm{~km} \mathrm{~S}$ of Riberalta, $11^{\circ} 08^{\prime}$ S, 66 $66^{\circ} 10^{\prime} W, 210$ m, 29-IX-1981, fl., Solomon 6515 (LPB, MO); La Paz: Mun. Guanay, V-1886, fl., Rusby 512 (F, GH, MO, NY, P). Santa Cruz: província Sara, bosque del rio Palometillas, 9-X-1924, fl., Steinbach 6606 (F, USZ); Mun. Santa Cruz de la Sierra, Angostura, margino of Río, 1-VII-1966, fl., Steinbach 339 (F, MICH, MO, NY, U, USZ); Mun. Santa Rosa Del Sara, Laguna Juan Chulo, 1659'13,7'S, 6344'46”'W, 260 m, 9-XI-2006, fr., Linneo 873 (MO, USZ). BRAZIL. Acre: Mun. Assis Brasil, Basin of Rio Acre, 1056'20'S, 69²9'51' W, 20-X-1997, fl. fr., Daly 9637 (MO, NY, UFACPZ); Mun. Bujari, Riozinho do Andirá, Ramal Nova Linha 1, ao longo da estrada, 9०42'59,4' $\mathrm{S}, 68^{\circ} 08^{\prime} 15,9^{\prime}$ 'W, 6-IX-2013, fl., Costa 271 (NY, RB); Mun. Jordão, Tarauca river, $09^{\circ} 14^{\prime} 19,8^{\prime}$ 'S, 71 ${ }^{\circ} 55^{\prime} 27,1$ ' $\mathrm{W}$, 5-II-2009, fl., Acevedo-Rodríguez 14780 (NY, RB); Mun. Rio Branco, road to Rio Branco, $33 \mathrm{~km}$ from the city, 29-IX-1980, fr., Lowrie 259 (INPA, MG, NY, R); Mun. Sena Madureira, Basin of Rio Iaco, Fazenda São Jorge I, ca. $22 \mathrm{~km}$ E on Toco Preto access road, 9²5'04"S, 68 36'45"'W, 8-VII-2008, fl., Daly 13263 (NY, RB, UFACPZ). Amazonas: rio Jau ad Belem, 12-V-1881, fl., Schwacke 3008 (RB). Pará: s.loc., s.dat., fl., Schwarz s.n. (W68981). PERU. s.loc., 5-X-1877, fl., Vidal-Senege s.n. (P06173021). Amazonas: Mun. Bagua, AramangoSalinas, $05^{\circ} 25^{\prime} 00$ "'S, 79³0'00”'W, 380 m, 6-XI-1999, fr., Rojas \& R. Vásquez 753 (HUT, MICH, MO, NY, USM); loc. cit., km 489 of Oleoducto nor Peruano, small stream near Mayo, $05^{\circ} 30^{\prime} \mathrm{S}, 7^{\circ} 30^{\prime} \mathrm{W}, 430-460 \mathrm{~m}$, 4-VI-1986, fr., Knapp 7575 (F, MO); loc. cit., Valley of Río Marañon, 05³0'S, 78³0'W, 430 m, 16-IV-1984, fr., Croat 58329 (MO, NY); loc. cit., road from La Peca-Bagua, 24-X-1978, fl., Barbour 4256 (MO).
Huánuco: Gauso Azul, rio Pachitea, IX-1942, fl., Vandeman 3309 (K). Junin: Mun. Satipo, road from San Ramon to Satipo, $39 \mathrm{~km}$ from La Merced, 1052'21' S, 7502'48”W, 589 m, 14-IX-2001, fl., Weigend 5735 (HUT, NY, SMF). Madre de Dios: Mun. Iberia, Vic. Río Ahuamanu, 6-IX-1945, fr., Seibert 2171 (MO); Mun. Manu, Cocha Cashu Camp, Río Manu, 18-X1979, fr., Gentry 26915 (AMAZ, MO, SFM); loc. cit., Parque Nacional Manú, Rio Cumerjali, 114'', 71³2’W, 350-450 m, 22-X-1986, fl., Foster 11965 (CUZ, F, INPA, K, US); Mun. Tambopata, Puerto Maldonado, Cuzco Amazónico, 130ㅇ'S, 69³6'W, 290-300 m, 26-XI-2002, fr., Valenzuela 1062 (CUZ, HUT, MO, USM); loc. cit., Las Piedras, Quebrada Loboyoc, 12²1'09's, 68 59'57' W, $161 \mathrm{~m}, 21$ X-2005, fl., Farfán 779 (AMAZ, CUZ, HUT, MO, MOL, P, USM); loc. cit., Campamento turistico Cusco Amazonico, zona 2, plot E, 12 $33^{\circ} \mathrm{S}, 69^{\circ} 03^{\prime} \mathrm{W}, 200 \mathrm{~m}$, 8-X-1998, fr., Vásquez 25843 (F, MO, NY); loc. cit., Las Piedras, Cusco Amazonico, 12²9'S, 6903'W, 200 m, 7-X-1991, fl. fr., Timaná 2427 (MO). Ucayali: Mun. Coronel Portillo, Tournavista, centro ganadero, margin of Río Pachitea, 16-III-1982, fl., Encarnación 26053 (G, MBM, MO, NY); loc. cit., 16-III-1982, fl., Encarnación 26055 (MO, NY); Mun. Purus, Cuenca del Rio Purus, Rio Curanja, ca. De la comunidad nativa de Colombiana, 300-350 m, 18-X-1997, fl., Graham 205 (MICH); loc. cit., 300-350 m, 12-II-2000, fl. fr., Graham 866 (MICH).

Distribution, habitat and phenology: Amorimia amazonica is known only to lowland Igarapé Forest in the Amazon basin from western Brazil, Bolivia and Peru (figure 33). Flowering from March to October and fruiting throughout the year.

Conservation status: Amorimia amazonica possesses a wide EOO of ca. $985,460.095 \mathrm{~km}^{2}$. Nonetheless, it possesses a restricted $\mathrm{AOO}$ (ca. $24.000 \mathrm{~km}^{2}$ ), intimately associated with Acre, Madeira, Purus, Mamore, Beni, Madre do Dios, Ucayali, Pachitea, and Halluaga river basins. Amorimia amazonica is mainly threatened by the deforestation of the Amazon Forest in Brazil (States of Acre and Rondônia), Bolivia, and Peru (WWF 2009). Thus, A. amazonica should be regarded as Endangered [EN, B1ab(iii,iv,v); C2a(i,ii); D2].

Etymology: the epithet makes reference to the restricted distribution of this species to the Amazon Forest.

Nomenclatural notes: Mascagnia amazonica, the basionym of Amorimia amazonica, was described 
by Niedenzu (1926) based on a single collection, Ule 9478, collected along the Acre River in Seringal of São Francisco do Iracema (currently the municipality of Xapuri), State of Acre, Brazil. According to Stafleu \& Cowan (1986), Niedenzu's specimens were generally housed at B, and were all destroyed during WWII. After consulting several herbaria, I found syntypes housed at G, K, L, MG, MO, and NY (the one at NY consisting of just two fragments). I designate the specimen at MG as the lectotype, since it is the most complete and best preserved, and also the only specimen housed in a Brazilian herbarium.

Taxonomic notes: Mascagnia amazonica Nied. was described only two years before the publication of the last revisionary monograph for Malpighiaceae (Niedenzu 1928). In addition to the original material listed in 1926, Niedenzu (1928) cited two additional collections for this species, one from the municipality of Baturite and the second from the municipality of Ipú, both in the State of Ceará, Brazil. Nonetheless, these collections occur in caatingas and rocky outcrops, divergent from the original material, which was collected in western Amazon Rainforests. About 80 years later, Anderson (2006), transfered $M$. amazonica to his new genus Amorimia, in order to accommodate all species from $M$. sect. Pleuropetrys. At this time, he also described two new species of Amorimia for Eastern Brazil, one of them, $A$. septentrionalis, perfectly matches those disjunct specimens cited by Niedenzu (1928) as part of $M$. amazonica, due to their pubescent anthers. Amorimia amazonica is morphologically related to A. camporum and $A$. kariniana, and can be distinguished from both by its sericeous-velutine indumenta on vegetative and reproductive organs, inflorescence bracts petiolate and lanceolate, and by sericeous-velutine samaras.

\subsection{Amorimia camporum W.R.Anderson, Novon} 16(2): 179.2006. Holotype: PERU. CAJAMARCA: Mun. San Ignacio, District Chirinos, entre La Catagua y Tablón, 5¹9'S, 7847’W, 550-650 m, 9-II-1996, fl., J. Campos \& O. Díaz 2490 (MICH barcode 1254384!; isotypes: F barcode 0092855!,MObarcode 1809916!, MO barcode 1809917!).

Figure 35

Woody vines to scandent shrubs; branches striated, sparsely lenticellate, lenticels whitish, sparsely velutine to glabrescent at age; stipules 0.3-0.5 mm long, narrowly triangular, interpetiolar on branches, epipetiolar on inflorescences, persistent to deciduous, velutine. Leaves opposite, reduced in inflorescences; petioles 2-6 mm long, canaliculate, sparsely velutine, eglandular to 2-glandular at apex; leaf blades 5-11 × 3-6.7 cm, plane, not bullate, elliptic to ovate, base cuneate to rounded, margin revolute, apex acute, obtuse, rounded, acuminate to apiculate, both sides velutine to glabrous at age, 1-many glands, 0.3-0.4 $\mathrm{mm}$ diam., up to $5 \mathrm{~mm}$ of margins; midvein adaxially impressed, abaxially prominent, 4-7 pairs of secondary veins, arching $45-50^{\circ}$, subopposite to alternate, adaxially impressed, abaxially prominent, reticulum conspicuous on both sides. Thyrsi (pseudoracemes) or panicles, axillary to terminal; main axis $5-18 \mathrm{~cm}$ long, flattened, smooth, velutine; cincinni 10-40, 1-flowered, decussate to spirally-alternate; reduced leaves wide-elliptic, apex mucronate; bracts $2-7 \times 0.7-2.5 \mathrm{~mm}$, ovate, plane, sessile, parallel to the peduncle, 1-2 pairs of glands at margins near base, both sides velutine; peduncle $2-11 \times 0.5-0.7 \mathrm{~mm}$, cylindrical, velutine; bracteoles $2.5-3 \times 0.4-0.7 \mathrm{~mm}$, ovate, plane with margins revolute, inserted at the apex of peduncles, opposite, spreading to the pedicel, eglandular, both sides velutine. Flowers $15-20 \mathrm{~mm}$ diam. at anthesis; floral buds $5-6 \times 3.5-4 \mathrm{~mm}$ at anthesis; pedicels 4-10 $\times$ 0.5-0.7 mm, cylindrical, velutine. Sepals $1-2 \times 1.5-1.7 \mathrm{~mm}$, triangular to ovate, appressed to the androecium, apex acute to obtuse, revolute at anthesis, adaxially glabrous, abaxially sericeous-velutine; glands yellow turning ocher at age, 1.8-3 × 1-1.3 mm. Petals yellow, margin sinuate, anterior-lateral petals not overlapping; lateral petals patent at anthesis, limb 5.5-8 $\times$ 4-6 mm, elliptic, obovate to orbicular, base cuneate, adaxially glabrous, abaxially sericeousvelutine; claws $1-2 \times 0.5-0.53 \mathrm{~mm}$, plane, adaxially glabrous, abaxially sericeous-velutine; posterior petal erect at anthesis, limb 5-7.5 $\times 3.5-5 \mathrm{~mm}$, orbicular, base cuneate, eglandular, adaxially glabrous, abaxially sericeous-velutine; claw 2-2.5 × 1-1.2 mm, plane, adaxially glabrous, abaxially sericeousvelutine. Stamens opposite petals longer than those opposite sepals; filaments $1.3-2.5 \times 0.35-0.40 \mathrm{~mm}$, connate ca. $0.2 \mathrm{~mm}$ long at base, glabrous; anthers monomorphic, straight, with a glandular connective, $0.8-1.1 \times 0.5 \mathrm{~mm}$, reflexed at anthesis, glabrous. Ovary 1.3-2 $\times 1.3-1.4 \mathrm{~mm}$, each carpel with primordial dorsal and lateral wings, velutine; styles 3, cylindrical at base, flattened at apex, parallel at base, divergent at middle, apex uncinate, sericeous-velutine at base,glabrous at middle and apex, anterior style ca. 1.4-1.45 mm long, posterior styles ca. 1.7-1.8 mm long; stigma lateral, crateriform. Samaras yellowish in vivo; dorsal wing 

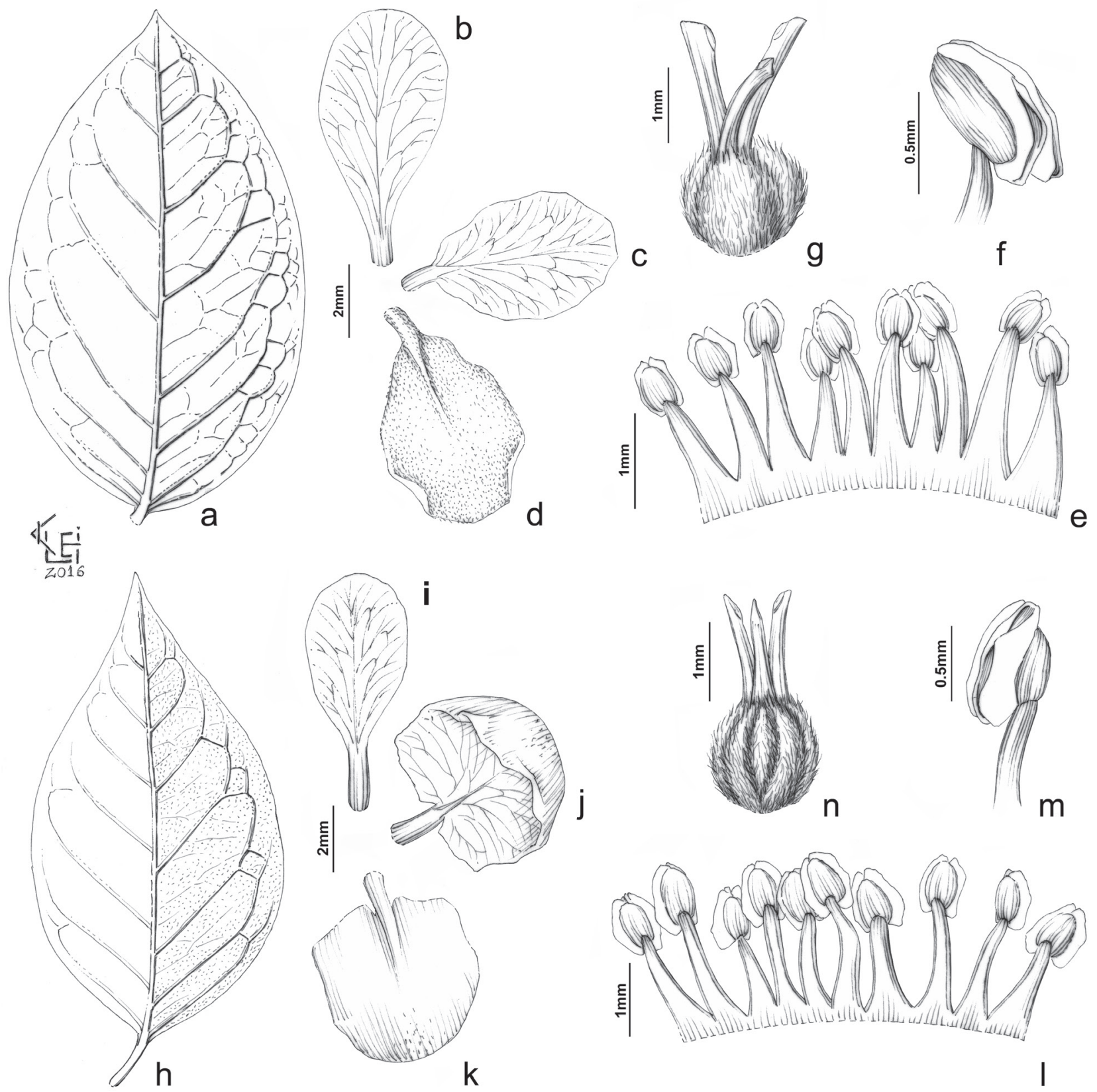

Figure 35. Amorimia camporum W.R. Anderson: a. abaxial side of a leaf evidencing vein pattern. b. adaxial side of a posterior petal. c. adaxial side of a posterio-lateral petal. d. abaxial side of a anterio-lateral petal. e. androecium. f. detail a stamen evidencing anther hairs. g. gynoecium (drawings by Klei Sousa). Amorimia concinna (C.V. Morton) W.R. Anderson: h. abaxial side of a leaf evidencing vein pattern. i. adaxial side of a posterior petal. j. adaxial side of a posterio-lateral petal. k. abaxial side of a anterio-lateral petal. 1. androecium. m. detail of a stamen evidencing anther hairs. n. gynoecium (drawings by Klei Sousa). 
3-6 $\times 1-2 \mathrm{~cm}$, depressed obovate, margin erose, plane, both sides velutine; lateral wings $1.4-3 \times 0.6-1.8 \mathrm{~cm}$, flabelliform, margin dentate, sinuate, upper angle $65^{\circ}$, lower angle $80^{\circ}$ from the nut, both sides sparsely velutine; nut 5-5.5 × 4-4.5 $\mathrm{mm}$, narrowly ovoid, velutine; areole $4.5-7 \times 2-3 \mathrm{~mm}$, narrowly ovate. Seeds $4.5-5 \times 2-3.5 \mathrm{~mm}$, testa smooth.

Specimens analyzed: PERU. Cajamarca: Distr. San Ignacio, Chirinos, 05'20'30'S, 78 46'00'W, 600-650 m, 6-I-1997, fl. fr., Campos 3266 (MO, NY); loc. cit., Chirinos, Las Juntas, s.dat., Campos 4846 (MICH); loc. cit., Distr. Huarango, entre Puerto Tabalozo y Nueva Esperanza, 550-700 m, 18-I-1996, Campos 2015 (HUT, MO); loc. cit., Puerto CirueloCamino a Huarango, s.dat., Campos 2658 (MICH). San Martín: Mun. Huallaga, entre Bellavista y Baños, s.dat., Ferreyra 4744 (MICH); Mun. Huinguillo, s.dat., Woytkowski 7183 (MICH, MO); Mun. Juan Jui, $5 \mathrm{~km}$ SE of Puente Colombia, 30-VI-1984, fl. fr., Murray 1531 (MO, NY); Mun. Saposoa, 656'S, 76² ${ }^{\circ}$ 'W, 450 m, 3-X-1959, fl., Woytkowski 5459 (F, MO, P); Mun. Tarapoto, along banks of Río Mayo, $06^{\circ} 23^{\prime}$ S, $76^{\circ} 39^{\prime} \mathrm{W}, 350$ m, 6-XI-1980, fr., Croat 51085 (MO); loc. cit., VI-1855, fl. fr., Spruce 4227 (F, MG, NY, P); loc. cit., road 10-25 km S of Tarapoto, $6^{\circ} 35^{\prime}$ 'S, $76^{\circ} 25^{\prime}$ W, 300-350, 18-VII-1982, fl. fr., Gentry 37660 (AMAZ, MO, NY, SMF); loc. cit., Valley of Rio Halluaga, $29 \mathrm{~km} \mathrm{~S}$ of Tarapoto, near El Abra, 6 40'S, 76²0'W, 450-540 m, 5-II-1984, fr., Gentry 44992 (MO); loc. cit., alto Río Huallaga, II-1936, Klug 4259 (K, MO, NY, US); loc. cit., Alto Rio Huallaga, 100 m, X-1934, fl., Klug 3881 (F, MO, NY); loc. cit., s.dat., Woytkowski 7200 \& 7202 (MICH, MO); Mun. Morales, Polvoraico, bosque secundario, $270 \mathrm{~m}$, 31-XII-1984, fr., Salas 418 (AMAZ).

Distribution, habitat and phenology: Amorimia camporum is known from Seasonally Dry Tropical Forests and Rainforests at 400-800 $\mathrm{m}$ in Cajamarca and San Martín, Peru (figure 33). Flowering and fruiting from December to April and in September.

Conservation status: Amorimia camporum is represented by only few records restricted to five municipalities within an EOO of ca. 135,981.058 km², and AOO of $20.000 \mathrm{~km}^{2}$, in SDTF within Mayo and Hualluaga river basins, in Peru. This species is mainly threatened by deforestation in the regions of Tarapoto and Iquitos. Thus, A camporum should be regarded as Endangered [EN, B1ab(iii,iv,v); C2a(i,ii); D2].
Etymology: the epithet pays honor to the collector of the type specimen, the Peruvian botanist José Ricardo Campos (b. 1955).

Taxonomic notes: Amorimia camporum is similar to $A$. amazonica and A. kariniana due to its leaf blades abaxially glandular, bracts lanceolate, usually biglandular near base, petals abaxially densely and evenly pubescent, and glabrous anthers. Amorimia camporum is especially similar to A. kariniana, due to their branches with whitish lenticels, stipules 0.3-0.7 mm long, petiole longer than $2 \mathrm{~mm}$ long, and samaras with areoles narrowly ovate to ovate to elliptic. Nonetheless, A. camporum can be differentiated by its larger leaves with petioles $6 \mathrm{~mm}$ long or shorter, sepals with revolute apex at anthesis, posterior petal only moderately differentiated from the lateral petals (the claw thicker and slightly longer, the limb somewhat smaller), limb of lateral petals 5.5-8 $\times 4-6 \mathrm{~mm}$, and samaras with lateral wings $14-30 \times 6-18 \mathrm{~mm}$ and dorsal wing usually extending at most to middle of nut, from there to base represented only by a rib.

\subsection{Amorimia concinna (C.V.Morton) W.R.Anderson,}

Novon 16(2): 180. 2006. Basionym: Mascagnia concinna C.V.Morton, Publ.Carnegie Inst. Wash.461: 130. 1936. 三 Mascagnia dumetorum C.V.Morton, Proc. Biol. Soc. Washington 45: 53. 1932. nom. illeg., non M. dumetorum Griseb., Abh. König1. Ges. Wiss. Göttingen 24: 67. 1879. Holotype: US barcode US00108446 $\uparrow$. Lectotype (designated here): COLOMBIA. Bolívar: Mun. Sincé, 25-I-1918, fl. fr., F.W. Pennell 4033 (NY barcode 00067653!; isolectotype: $\mathrm{GH}$ barcode 00045140 !).

\section{Figure 35}

Woody vines to scandent shrubs; branches striated, densely lenticellate, lenticels whitish, sparsely sericeous-velutine to glabrescent at age; stipules 1-1.2 mm long, narrowly triangular, glabrous, interpetiolar on branches, epipetiolar on inflorescences, persistent to deciduous. Leaves opposite, reduced in inflorescences; petioles 8-9.5 mm long, canaliculate, densely sericeous-velutine, eglandular to 2-glandular at apex; leaf blades 7.1-10.7 $\times 3.5-6 \mathrm{~cm}$, plane, bullate, wide elliptic to ovate, base cuneate to rounded, margin plane, apex long acuminate to short mucronate, both sides sparsely to densely velutine to glabrescent at age, 1 pair of marginal glands at margins, 0.4-0.5 mm diam.; midvein adaxially impressed, abaxially prominent, 5-8 pairs of secondary veins, arching $45-50^{\circ}$, subopposite to alternate, adaxially 
impressed, abaxially prominent, reticulum adaxially inconspicuous, abaxially conspicuous. Thyrsi (pseudoracemes) or panicles, axillary to terminal; main axis $4-5.5 \mathrm{~cm}$, flattened, smooth, velutine; cincinni 10-12, 1-flowered, decussate; reduced leaves elliptic, apex acuminate; bracts 3.9-4.5 $\times 0.8-1 \mathrm{~mm}$, lanceolate, filiform, plane, sessile, parallel to the peduncle, eglandular, adaxially glabrous, abaxially velutine; peduncle 4-4.5 × 0.5-0.6 mm, cylindrical, velutine; bracteoles $2.5-3 \times 0.4-0.5 \mathrm{~mm}$, lanceolate, filiform, plane, sessile, inserted at the apex of peduncles, opposite, parallel to the pedicel, eglandular, adaxially glabrous, abaxially velutine. Flowers $1.4-1.5 \mathrm{~mm}$ diam. at anthesis; floral buds 7-8 $\times 5-6 \mathrm{~mm}$ at anthesis; pedicels 5-9 $\times$ 0.5-0.6 mm, cylindrical, velutine. Sepals 3.7-4 × 2-2.1 mm, triangular, appressed to the androecium, apex acute, revolute at anthesis, adaxially glabrous, abaxially sericeous-velutine; glands greenish to yellow, 2.2-2.5 $\times 1-1.25 \mathrm{~mm}$. Petals yellow, cucullate, margin sinuate, anteriorlateral petals not overlapping; lateral petals patent at anthesis, limb 5.5-6 $\times$ 4.5-5.5 mm, wide elliptic to obovate, base cuneate, adaxially glabrous, abaxially sparsely sericeous; claws 1-2 × 0.5-0.55 mm, plane, both sides glabrous; posterior petal erect at anthesis, limb 5.5 × 3-3.2 mm, elliptic, base cuneate, eglandular, adaxially glabrous, abaxially sparsely sericeous; claw 2-2.5 $\times 1-1.2 \mathrm{~mm}$, plane, both sides glabrous. Stamens opposite petals shorter than those opposite sepals; filaments 3-4 × 0.35-0.4 mm, connate $0.15-0.2 \mathrm{~mm}$ long at base, glabrous; anthers monomorphic, straight, with a glandular connective, 0.9-1 $\times 0.5-0.55 \mathrm{~mm}$, erect at anthesis, glabrous. Ovary 2-2.5 $\times 2-2.5 \mathrm{~mm}$, each carpel with primordial dorsal and lateral wings, velutine; styles 3 , cylindrical at base, flattened at apex, divergent at base, apex apiculate, base velutine, middle and apex glabrous, anterior style 1.8-2 mm long, posterior styles 2.1-2.2 mm long; stigma lateral, crateriform. Samaras yellowish in sicco; dorsal wing 1-1.1 × 0.35-4 cm, depressed obovate, margin dentate, sinuate, both sides velutine; lateral wings $1.5-2 \times 2.1-2.1 \mathrm{~cm}$, obdeltate, margin erose, sinuate, upper angle $30^{\circ}$, lower angle $40^{\circ}$ from the nut, both sides velutine; nut $3.2-4.5 \times 2.2-2.5 \mathrm{~mm}$, narrowly ovoid, velutine; areole 3-3.2 $\times 1.9-2 \mathrm{~mm}$, ovate. Seeds 3.8-4 $\times 3-3.3 \mathrm{~mm}$, testa smooth.

Specimens analyzed: COLOMBIA. Bolívar: Mun. Magangué, Corregimiento de Juan Arias, hacienda Valle María, propriedad de Gonzalo Botero, 19-II-1958, fr., Botero s.n. (COL52205); Mun. San
Pedro, 30 m, XII-1956, fl. fr., Arteta s.n. (COL50614); Mun. Sincelejo, road entre Sincelejo y Colosó, XII-1962, fl., Castañeda 9292 (COL, NY); loc. cit., XI-1962, fl. fr., Castañeda 9252 (COL, NY); loc. cit., 20-IV-1963, fl. fr., Castañeda 9646 (COL, NY). Magdalena: Mun. Fundación, km 3 de la carretera a Valledupar, Hacienda Córdoba de F. García \& Cía, VIII-1964, fr., Salas 1 (COL); loc. cit., Hacienda da Córdoba, situada al pie de la ciudad, XII-1963, fl., Castañeda 10040(COL); Mun. Pivijay, 6-IV-2006, fl., Mojica 1 (COL); Mun. Valledupar, roadside, $150 \mathrm{~m}$, 12-I-1944, fl. fr., Haught 3927 (COL, F, US). Sucre: Mun. Cince, VII-1997, fl., Uribe s.n. (COL423253).

Distribution, habitat and phenology: Amorimia concinna is known only from SDTF from the departments of Bolívar and Magdalena in northern Colombia (Figure 33). It is mostly restricted to the Magdalena river basin to Valledupar near the borders with Venezuela. Flowering from November to January and fruiting from August to February.

Conservation status: Amorimia concinna is represented by only few records restricted to five municipalities within an EOO of $11,867.658 \mathrm{~km}^{2}$ and AOO of $16.000 \mathrm{~km}^{2}$ in anthropically modified SDTFs. Amorimia concinna is greatly threatened by deforestation due to banana, coffee, cocoa, cassava and cotton crops, cattle breeding and farming in Colombia. Thus, it should be regarded as Endangered [EN, B1ab(iii,iv,v); C2a(i,ii); D2].

Etymology: the epithet means "elegant" and makes reference to the beautiful and elegant aspect of this species in vivo.

Nomenclatural notes: in January 2015, during a visit to the US herbarium, I was unable to locate the holotype of M. concinna. This specimen was previously loaned to the late Dr. William R. Anderson (MICH) for his studies in Mascagnia s.l. and the publication that resulted in the description of Amorimia, together with seven additional segregates (Anderson 2006). Before loaned, this type specimen was digitalized and an image of it is found in the US herbarium website (https://collections.nmnh. si.edu/search/botany/?ark=ark:/65665/369108a94cb 7b4331a995fa54d1210c08). In 2008, the specimen was returned to the US by the staff from University of Michigan herbarium (Christiane Anderson, pers. comm.), but was never received by the herbarium (John Boggan, US herbarium type collection curator, pers. comm.). During the following years, there have been several attempts from both herbaria (i.e., US and $\mathrm{MICH}$ ) to locate and retrieve the lost holotype. 
Nonetheless, they have all been unsuccessful. Thus, in accordance to the Code (McNeill et al. 2012, Art. 9.12), I designate one of the still extant isotypes as the lectotype of $M$. concinna. If the holotype is ever recovered, the herein designated lectotypification is to be superseded (McNeill et al. 2012, Art. 9.19).

Taxonomic notes: Amorimia concinna resembles $A$. pubiflora due to their similar fruit morphology, and because they are the only two species from $A$. subg. Uncinae that possess densely velutine indumentum in several vegetative structures. Amorimia concinna is also similar to $A$. kariniana due to its pubescent anthers, being differentiated by its marginal leaf glands, bracts linear and eglandular, and petals only sparsely sericeous abaxially in center of limb, otherwise glabrous.

\subsection{Amorimia kariniana W.R.Anderson, Novon} 16(2): 180. 2006. Holotype: ECUADOR. Guayas: Mun. Guayaquil, Cerro Azul, 50 m, 22-X-1958, fl., $G$. Harling 3026 (S16-19915!; isotype: MICH barcode 1244753 !).

Figure 36

Woody vines to scandent shrubs; branches striated, sparsely lenticellate, lenticels whitish, sparsely sericeous-velutine to glabrescent at age; stipules 0.3-0.7 $\mathrm{mm}$ long, narrowly triangular, interpetiolar on branches, epipetiolar on inflorescences, persistent to deciduous. Leaves opposite, not reduced in inflorescences; petioles $5-10 \mathrm{~mm}$ long, canaliculate, sparsely sericeous-velutine to glabrous at age, eglandular to biglandular at apex; leaf blades 8.5-13 $\times 5-8.5 \mathrm{~cm}$, plane, not bullate, wide-elliptic, base cuneate to rounded, margin plane, apex shortacuminate, both sides velutine to glabrescent, eglandular to 1-many pairs of glands, $0.25-0.28 \mathrm{~mm}$ diam., up to $3 \mathrm{~mm}$ of margins; midvein adaxially impressed, abaxially prominent, 5-7 pairs of secondary veins, arching $35^{\circ}$, subopposite to alternate, adaxially impressed, abaxially prominent, reticulum prominent on both sides. Thyrsi (pseudoracemes) or panicles, axillary to terminal; main axis $7-18 \mathrm{~cm}$ long, flattened, smooth, velutine; cincinni 16-26, 1-flowered, decussate; reduced leaves absent; bracts 3-6 $\times 1-3 \mathrm{~mm}$, lanceolate, plane, sessile, parallel to the peduncle, 1 pair of marginal glands at base, both sides velutine; peduncle 2-7 × 0.5-0.6 mm, cylindrical, velutine; bracteoles 2-2.3 $\times 0.3-0.4 \mathrm{~mm}$, lanceolate to filiform, plane with revolute margins, sessile, opposite, inserted at the apex of peduncles, parallel to the pedicel, eglandular, both sides velutine. Flowers 2-2.2 mm diam. at anthesis; floral buds 6-7 $\times 6-7 \mathrm{~mm}$ at anthesis; pedicels $6-9 \times 0.5-0.6 \mathrm{~mm}$, cylindrical, velutine. Sepals $1.5-2 \times 1.7-2.5 \mathrm{~mm}$, widely ovate, appressed to the androecium, apex acute to obtuse, revolute at anthesis, adaxially glabrous, abaxially sericeous-velutine; glands yellowish, 2.5-3.5 × 1-1.2 mm. Petals yellow, margin sinuate, anterior-lateral petals not overlapping; lateral petals patent, limb 7.5-8.5 × 6-7 $\mathrm{mm}$, wide obovate, base cuneate, adaxially sparsely velutine to glabrous distally, abaxially sericeous-velutine; claws 1.5-2 × 0.5-0.6 mm, plane, adaxially glabrous, abaxially sericeous-velutine; posterior petal erect, limb 6-6.5 × 4.5-5 mm, spatulate, base cuneate, eglandular, adaxially sparsely velutine to glabrous distally, abaxially sericeous-velutine; claw 3.5-4 × 1-1.3 mm, plane, adaxially glabrous, abaxially sericeous-velutine. Stamens opposite petals shorter than those opposite sepals; filaments 2.5-3.5 × 1-1.25 mm, connate ca. $0.2 \mathrm{~mm}$ long, glabrous or abaxially sparsely sericeous; anthers monomorphic, straight, with a glandular connective, $0.8-1.4 \times 0.5-0.7 \mathrm{~mm}$, reflexed in anthesis, glabrous. Ovary 1.7-2 × 1.3-1.5 mm, each carpel with primordial dorsal and lateral wings, velutine; styles 3 , cylindrical at base, laterally flattened at apex, parallel at base, divergent at middle, apex uncinate, base sericeous-velutine, anterior style 1.8-2 mm long, posterior styles 2.1-2.2 mm long; stigma lateral, crateriform. Samaras yellowish in vivo; dorsal wing 12-15 $\times$ 4-5 mm, depressed obovate, margin erose, sinuate, both sides velutine; lateral wings 3-4.2 $\times 2-3 \mathrm{~cm}$, flabelliform, margin erose, sinuate, upper angle $70^{\circ}$, lower angle $80^{\circ}$ from the nut, both sides velutine; nut $8-12 \times 4-6 \mathrm{~mm}$, narrowly ovoid, velutine; areole 8-12 $\times 4-6 \mathrm{~mm}$, ovate to elliptic. Seeds ca. $8 \times 5 \mathrm{~mm}$, testa smooth.

Specimens analyzed: ECUADOR. Guayas: Mun. Guayaquil, Cerro Azul, s.dat., fl., Asplund 16617, 17586 (S); loc. cit., road to Aguas Piedras, s.dat., fl. fr., Owlee 1109 (US); loc. cit., Cerro Mirador de los Monos, s.dat., fl., Rubio 2445 (MICH, MO); Mun. Pedro Carbo, along a stream, 8-VII-1940, fl., Haught 3070 (MICH, NY, US). Santa Elena: E of Las Juntas, s.dat., fl., Fagerlind 242 (S).

Distribution, habitat and phenology: Amorimia kariniana is known only from lowland SDTF from Guayas, Ecuador (figure 33), along streams, roadside thickets, and pastures. Flowering from June to October, and fruiting from February to June.

Conservation status: Amorimia kariniana is represented by few records, restricted to an EOO of 1,215.151 km² 
and $\mathrm{AOO}$ of $12.000 \mathrm{~km}^{2}$ in anthropically modified STDFs in Guayas, Ecuador. Amorimia kariniana is highly threatened by sugar refinaries, iron foundries, tanneries and sawmills, but also by deforestation for the development of banana, cocoa and coffee crops. Thus, it should be regarded as Endangered [EN, B1ab(iii,iv, v); C2a(i,ii); D2].
Etymology: The epithet pays honor to Karin Weishaar Douthit (b. 1926), a remarkable and long-time plant illustrator from the University of Michigan.

Taxonomic notes: Amorimia kariniana resembles $A$. amazonica, A. camporum and $A$. concinna due to their pubescent anthers. Amorimia kariniana is especially similar to A. camporum, due to their branches with
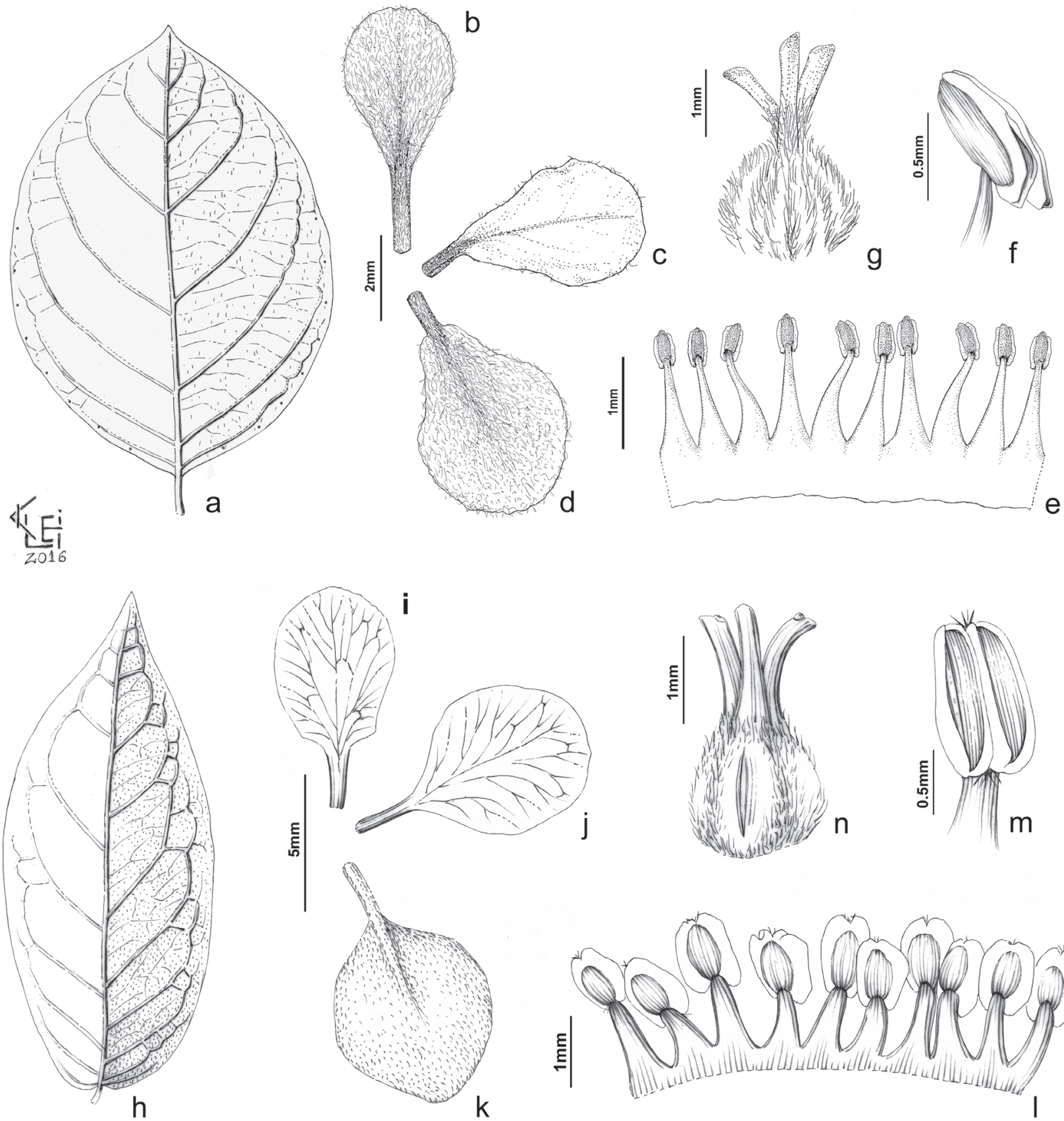

Figure 36. Amorimia kariniana W.R. Anderson: a. abaxial side of a leaf evidencing vein pattern. b. adaxial side of a posterior petal. c. adaxial side of a posterio-lateral petal. d. abaxial side of an anterio-lateral petal. e. androecium. f. detail of a stamen evidencing anther hairs. g. gynoecium (drawings by Klei Sousa and Karin Weishaar Douthit). Amorimia pubiflora (A. Juss.) W.R. Anderson: h. abaxial side of a leaf evidencing vein pattern. i. adaxial side of a posterior petal. $\mathrm{j}$. adaxial side of a posterio-lateral petal. $\mathrm{k}$. abaxial side of a anteriolateral petal. 1. androecium. m. detail of a stamen evidencing anther hairs. n. gynoecium (drawings by Klei Sousa). 
whitish lenticels, stipules $0.3-0.7 \mathrm{~mm}$ long, petiole longer than $2 \mathrm{~mm}$ long, and samaras with areoles narrowly ovate to ovate to elliptic. Nonetheless, it can be differentiated by its larger leaves with petioles (5-)7-10 mm long, sepals mostly appressed at anthesis, posterior petal strongly differentiated from lateral petals (with a much longer claw and smaller limb), limb of lateral petals 7.5-8.5 $\times 6-7 \mathrm{~mm}$, and samaras with lateral wings $3-4.2 \times 2-3 \mathrm{~cm}$ and with the dorsal wing extending to base of nut or nearly so.

\subsection{Amorimia pubiflora (A.Juss.) W.R.Anderson,} Novon 16(2): 183.2006. Basionym: Hiraea pubiflora A.Juss., Fl. Bras. Merid. (quarto ed.) 3(21): 14.1832 [1833]. झ Mascagnia pubiflora (A.Juss.) Griseb., Fl. Bras. 12(1): 91. 1858. 三 Mascagnia pubiflora var. typica Nied., Arbeiten Bot. Inst. Königl. Lyceums Hosianum Braunsberg 3: 23. 1908. nom. illeg. Lectotype (designated by Anderson 2006): BRAZIL. Minas Gerais: near Mangahy (currently Municipality of Manga), 1816, fl. fr., A. St.-Hilaire s.n. (P barcode 02429221!; isolectotypes: MPU barcode 020182!, P barcode 02429220 !, P barcode 02429222 !).

= Mascagnia parnahybensis Glaziou, Bull. Soc. Bot. France 52(Mém. 3a): 77. 1905. 三 Mascagnia pubiflora var. grandifolia Nied., Arbeiten Bot. Inst. Königl. Lyceums Hosianum Braunsberg 3: 23. 1908. nom. illeg. $\equiv$ Mascagnia pubiflora var. parnahybensis (Glaziou) Nied., Pflanzenr. IV. 141 (Heft 91): 115. 1928. Lectotype (designated by Anderson 2006): BRAZIL. GoIÁs: border of Rio Parnahyba, I-1894, fl. fr., A. Glaziou 20757 (P barcode 02429219!; isotypes: G barcode 00352760!, K barcode 000427419!, P barcode 02429218 !).

Figures 36-37

Woody vines to scandent shrubs; branches striated, sparsely lenticellate, lenticels brown, densely velutine to glabrescent at age; stipules 0.4-0.5 mm long, triangular, glabrous, interpetiolar on branches, epipetiolar on inflorescences, persistent to deciduous. Leaves opposite to subopposite, reduced in inflorescences; petioles 3-7 mm long, canaliculate, densely velutine to glabrous at age, eglandular to biglandular at apex; leaf blades 7.5-20.5 $\times 4-12 \mathrm{~cm}$, plane, not bullate, elliptic to ovate, base cordate, rarely cuneate, margin revolute, apex acuminate to mucronate, both sides densely velutine to glabrescent at age, eglandular to 1 pair of glands, $0.3-0.5 \mathrm{~mm}$ diam., near base or up to $2.5 \mathrm{~mm}$ of margins; midvein adaxially impressed, abaxially prominent,
7-9 pairs of secondary veins, arching $45-50^{\circ}$, subopposite to alternate, adaxially bullate, abaxially prominent, reticulum prominent on both sides. Thyrsi (pseudoracemes) or panicles, axillary; main axis 12-32 cm long, slightly flattened, striated, densely velutine; cincinni 50-52, 1-flowered, decussate to alternate; reduced leaves elliptic, apex mucronate; bracts $4.5-5.5 \times 1.5-2.5 \mathrm{~mm}$, elliptic to lanceolate, plane, petiolate, leaf-like, parallel to peduncle, 1 pair of glands at base to middle, both sides velutine; peduncle 7-8.5 × 0.7-1.2 mm, cylindrical, velutine; bracteoles 3-3.5 $\times 1-2 \mathrm{~mm}$, elliptic to lanceolate, plane, petiolate, opposite to subopposite inserted bellow the apex of peduncles, parallel to the pedicel, 1 pair of basal glands, both sides velutine. Flowers 2-2.8 mm diam. at anthesis; floral buds 5.5-7 $\times 4$.8$5.3 \mathrm{~mm}$ at anthesis; pedicels $8-12 \times 1-1.2 \mathrm{~mm}$, cylindrical, velutine. Sepals 3-3.5 $\times 1.5-2 \mathrm{~mm}$, widely ovate, not appressed to the androecium, apex acute to obtuse, revolute at anthesis, adaxially glabrous, abaxially velutine; glands green turning yellow, 1.7-2.5 $\times 1-1.5 \mathrm{~mm}$. Petals yellow, margin sinuate, anterior-lateral petals not overlapping; lateral petals patent, limb 7-10 × 5.5-7.5 mm, elliptic to spatulate, base cuneate to obtuse; claws $2-2.5 \times 0.5-0.55 \mathrm{~mm}$, plane, adaxially glabrous, abaxially velutine; posterior petal erect, limb 8-8.5 $\times 3.5-5.5 \mathrm{~mm}$, elliptic, base rounded, eglandular to 1-pair of glands, adaxially glabrous, abaxially velutine; claw 3.5-3.7 × 1-1.2 mm, plane, adaxially glabrous, abaxially velutine. Stamens opposite petals longer than those opposite sepals, those opposite the anterior-lateral sepals partially or completely fused to the adjacent filament; filaments 1.5-2.5 × 0.3-0.5 mm, connate ca. $0.1 \mathrm{~mm}$ long at base, filaments opposite to sepals larger than those opposite to petals shorter, glabrous; anthers monomorphic, straight, with a glandular connective, 1.5-2.5 $\times 0.3-0.5 \mathrm{~mm}$, erect to reflexed, pubescent at base and apex. Ovary 1.5-2 $\times 1.5-2 \mathrm{~mm}$, each carpel with primordial dorsal and lateral wings, velutine; styles 3, cylindrical at base, laterally flattened at apex, parallel at base, divergent at middle, apex uncinate, pubescent at base, glabrous at middle and apex, anterior style 1.2-1.8 mm long, posterior styles 1.5-2 mm long; stigma lateral, crateriform. Samaras bright yellow in vivo; dorsal wing 13-14 × 3-4 mm, depressed obovate, margin entire, sinuate, both sides velutine; lateral wings 1.8-2 × 2.7-3.2 cm, flabelliform, margin erose, sinuate, upper angle $55-60^{\circ}$, lower angle $60^{\circ}$ from the nut, both sides velutine; nut 9-9.5 $\times 3.5-4 \mathrm{~mm}$, 
ovoid, velutine; areole 9.4-10 × 3-3.5 mm, narrowly triangular. Seeds 7-8 $\times 5-5.5 \mathrm{~mm}$, testa smooth.

Specimens analyzed: BRAZIL. Without PROVINCE: sin. loc., s.dat., fl., Pohl s.n. (F872521); sin. loc., s.dat., fl., s.leg. 1545 (KH2013/0134821). Bahia: Porto, extremo Rio São Francisco, 27-VII-1939, fl., Mendes s.n. (HUEFS 204341, SP); loc. cit., 27-VII-1939, fr., Mendes s.n. (HUEFS 204340, SP). Goiás: Mun. Piranhas, disturbed hillside, 700 m, 22-VI-1966, fl., Irwin 17573 (IAN, NY, UB). Mato Grosso: Mun. Cáceres, VI-2011, fl., Molleta s.n. (UFMT); loc. cit., 24-VII-1972, fl., Jesus 1745 (RB); Mun. Colniza, 16-VII-2012, fl. fr., Pereira s.n. (UFMT43014). Mato Grosso do Sul: Mun. Aquidauana, nos arredores de Aquidauana, IV-2001, fl., Garcez s.n. (CGMS, HUEFS207597); loc. cit., Fazenda Santa Cruz, 17-VII-1969, fl. fr., Hatschbach 21887 (MBM, P); loc. cit., Fazenda Cutepe, 6-IX-1971, fl., Döbereiner 803 (F, RB, US); Mun. Bodoquena, local próximo a sede da fazenda Rancho Branco, entorno leste do Parque Nacional da Serra de Bodoquena, 20 40'15"S, $56^{\circ} 45^{\prime} 55^{\prime}$ 'W, 230 m, 30-VIII-2005, fl. fr., Pott 13270 (CGMS, HMS, HUEFS); Mun. Brasilândia, entre Lagoa Sucuri-Duzão, 5205'S, $21^{\circ} 43^{\prime}$ W, 25-IX-1996, fr., Rezende 3556 (SPF, SJRP); Mun. Miranda, local sede da Fazenda Miranda, 4-IX-2000, fl. fr., Pott 4204 (CGMS, HMS, HUEFS, SP); loc. cit., Fazenda Lacobe, IV-1971, fl., Döbereiner 795 (HUEFS, RB); Mun. Três Lagoas, local Chamflora, margem do rio Paraná,
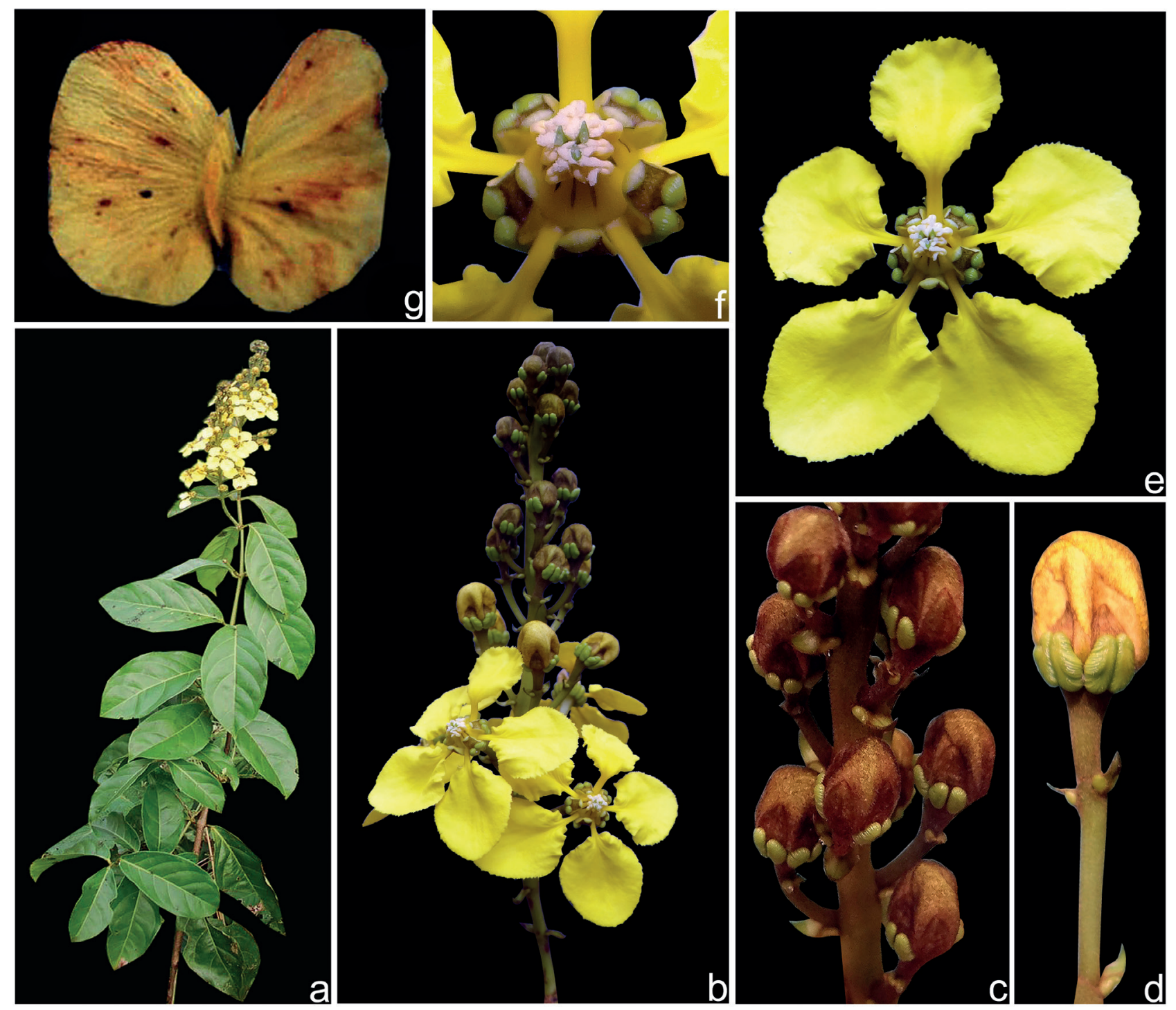

Figure 37. Amorimia pubiflora (A. Juss.) W.R. Anderson: a. flowering branch. b. inflorescence and flowers. c. floral buds with ocher indumenta. d. floral bud showing bract and bracteoles. e. flower in frontal view. f. detail of the androecium and gynoecium. g. samara in frontal view (photographs by E. Moletta). 
2100'55"S, 51 45'39”'W, 260 m, 27-IX-2002, fl. fr., Pott 10411 (CEN, CGMS, CPAP, HMS, HUEFS, SP); loc. cit., Porto Independência, 20-X-1994, fl., Jacques 357 (CGMS, UB); loc. cit., Lagoa Funda, 18-IV-1971, fl., Döbereiner 774 (HUEFS, RB); loc. cit., Barra do Moeda, Reserva Florestal de Três Lagoas Agroflorestal Ltda, 18-VI-1993, fl., Caliente 609 (HISA, SP); loc. cit., 27-VIII-1955, fl., Ribeiro s.n. (IAC18036). Minas Gerais: Jiquetahy in Rio São Francisco, s.dat., fl., Pohl 5778 (W0068976!; isolectotype: NY!; Mun. Araporã, Reserva Vegetal da Usina Alvorada, 12-X-2000, fr., Arantes s.n. (HUEFS211196, HUFU28411); loc. cit., 7-VI-2001, fr., Arantes s.n. (HUEFS 211197, HUFU); Mun. Itacarambi, s.dat., fr., Ferreira s.n. (PAMG11405); Mun. Jaíba, APA Serra do Sabonetal, Fazenda Agropeva, 21-IX-2006, fl. fr., Marino 203 (BHCB); Mun. Januária, fazenda Santa Cecília, área de pastagem, 17-VIII-1994, fl., Arbocz 656 (HUEFS, SP); loc. cit., Vale do Peruaçu, Janelão, 25-X-1997, fl. fr., Salino 3703 (BHCB); loc. cit., vale do Peruaçu, perto do Boqueirão, 1507'23,9' $S$, 4414'29'W, s.dat., fl., Costa 363 (BHCB56397); Mun. Santa Luzia, Fazenda São Sebastião, 26-X-1938, fr., Malheiros s.n. (HUEFS204343, SP39746); Mun. Tupaciguara, Fazenda Córrego Fundo, 1-IX-1972, fl., Döbereiner 901 (BHCB, PAMG, RB); Mun. Uberlândia, entre Uberlândia e Monte Alegre, 15-I-1980, fl. fr., Parreira 1 (PAMG). São Paulo: sin. loc., 16-VIII- 1960, fl., Silva s.n. (IAC18641); sin. loc., X-1961, fr., Toledo s.n. (IAC18708); Mun. Andradina, Fazenda Guanabara, 13-XII-1939, fl., Rocha-Silva s.n. (SP012033); Mun. Araraquara, 20-IX-1961, fl., Vieira s.n. (HUEFS203425, RB315272, SPF23655); Mun. Castilho, Estação Junqueira, Fazenda Itapura, 19-IX-1941, fl., Corbett s.n. (HUEFS204337, SP45967); loc. cit., entre estação Junqueira e Porto Jupiá, rio Paraná, 22-VII-1961, fl. fr, Jaccoud 68 (SP); loc. cit., 20-VIII-1972, fl., Melichenko s.n. (IAC23057); Mun. Panorama, bairro de Itaziara, rio Paraná, 13-X-1998, fr., Bicudo 88 (SP); Mun. Pereira Barreto, 12-VIII-1938, fl., Lara s.n. (HUEFS 204344, $\mathrm{SP})$.

Distribution, habitat and phenology: Amorimia pubiflora is known to semi-deciduous forests in the States of Bahia, Mato Grosso, Mato Grosso do Sul, Minas Gerais, and São Paulo, Brazil (figure 33). Flowering from April to December and fruiting from June to October.

Conservation status: Amorimia pubiflora originally possessed a wide EOO of ca. 1,507,628.658 $\mathrm{km}^{2}$. It has been intensively removed from its natural habitat by farmers, since it is the main cause of bovine sudden death in central Brazil. Farmers employ a wide range of control techniques, such as biological, manual and mechanical, and have been able to almost completely eradicate the species in some regions. This is supported by our complete lack of success in collecting A. pubiflora in the past five years, in different parts of Brazil, where the species was once considered common. Thus, it should be regarded as Critically Endangered [CR, A3b; B1ab(v); C2a(i,ii); D1; E].

Etymology: the epithet makes reference to its densely pubescent floral buds (figure 37C).

Taxonomy notes: A. pubiflora resembles A. concinna due to their similar fruit morphology, and because they are the only two species from A. subg. Uncinae that possess densely velutine indumentum in several vegetative organs. Amorimia pubiflora is also similar to $A$. septentrionalis due to its glabrous anthers, being easily differentiated by its velutine stems, without lenticels, leaf blades velutine, cordate or subcordate at base, and petals adaxially glabrous.

2.6. Amorimia septentrionalis W.R.Anderson, Novon 16(2): 183. 2006. Holotype: BRAZIL. Ceará: Sin. loc., 22-VI-1958, fl. fr., J. Döbereiner 538 (MICH barcode 1244752 !).

Figures 38, 40

Woody vines to scandent shrubs; stem striated, densely lenticellate, lenticels whitish, sparsely tomentose to glabrescent at age; stipules $0.5-1.5 \mathrm{~mm}$ long, narrowly triangular, glabrous, interpetiolar on branches, epipetiolar on inflorescences, persistent to deciduous. Leaves opposite, reduced in inflorescences; petioles 3-4 mm long, canaliculate, sparsely sericeousvelutine to glabrous at age, eglandular; leaf blades $5-12 \times 2.5-5.5 \mathrm{~cm}$, plane, not bullate, narrow to wideelliptic to obovate, base cuneate to rounded, margin plane, apex acute to acuminate to rounded, adaxially sericeous-velutine to glabrescent at age, abaxially persistently sericeous-velutine, eglandular to 1 pair of glands, 0.3-0.5 mm diam., near base or up to $1.4 \mathrm{~mm}$ of margins; midvein adaxially impressed, abaxially prominent, 4-6 pairs of secondary veins, arching $50^{\circ}$, subopposite to alternate, adaxially impressed, abaxially prominent, reticulum prominent on both sides. Thyrsi (pseudoracemes) to panicles, axillary to terminal; main axis 4-20 cm long, slightly flattened, striated, sericeous-velutine; cincinni 10-50, 1-flowered, 
decussate to alternate; reduced leaves elliptic to obovate, apex mucronate; bracts 2-6 × 1-2 mm, lanceolate, plane, sessile, leaf-like, parallel to the peduncle, 1 pair of glands at base, both sides sericeousvelutine; peduncle $1-4 \times 0.4-0.5 \mathrm{~mm}$, cylindrical, sericeous-velutine; bracteoles 1.2-1.8 $\times 0.6-1 \mathrm{~mm}$, elliptic, plane with revolute margins, sessile, inserted at the apex or below peduncles, parallel to the pedicel, eglandular to 1 pair of glands at base, both sides sericeous-velutine. Flowers 0.9-1.1 mm diam. at anthesis; floral buds 4.5-5 × 3-3.5 mm at anthesis; pedicels 3-4 × 0.4-0.5 mm, cylindrical, sericeous-velutine. Sepals 1.5-2.2 × 1.2-2 mm, triangular, not appressed to the androecium, apex acute, revolute at anthesis, adaxially glabrous, abaxially sericeous-velutine; glands yellow turning ocher, 6-10 $\times 2-2.8 \mathrm{~mm}$. Petals yellow turning orange, margin sinuate, anterior-lateral petals not overlapping; lateral petals patent at anthesis, limb 3.2-6 $\times 2-2.7 \mathrm{~mm}$, elliptic to spatulate, base cuneate, adaxially sericeousvelutine distally, abaxially sericeous-velutine; claws 0.5-1.2 $\times 0.5-0.55 \mathrm{~mm}$, plane, adaxially sericeousvelutine, abaxially sericeous-velutine; posterior petal erect, limb 3.3-4.2 × 2-2.5 mm, spatulate, base cuneate, eglandular, adaxially sericeousvelutine distally, abaxially sericeous-velutine; claw 0.7-1.5 × 1-1.2 mm, plane, adaxially sericeousvelutine, abaxially sericeous-velutine. Stamens opposite petals longer than those opposite sepals; filaments $1.5-2 \times 0.4-0.5 \mathrm{~mm}$, connate $0.15 \mathrm{~mm}$ long at base, glabrous; anthers monomorphic, recurved, with a glandular connective, 0.8-1.5 $\times 0.5-0.57 \mathrm{~mm}$, erect at anthesis, pubescent at base and apex. Ovary $1.2-1.5 \times 1.2-1.5 \mathrm{~mm}$, each carpel with primordial dorsal and lateral wings, sericeous-velutine; styles 3, cylindrical at base, laterally flattened at apex, parallel at base, divergent at middle, apex uncinate, sericeousvelutine at base, glabrous at middle and apex, anterior style 1.4-1.42 mm long, posterior styles 1.5-1.6 mm long; stigma lateral, crateriform. Samaras green to ocher in vivo; dorsal wing 7-15 $\times 1.5-5 \mathrm{~mm}$, depressed obovate, margin erose, sinuate, both sides sericeousvelutine; lateral wings $2-3.3 \times 0.8-2 \mathrm{~cm}$, flabelliform, margin dentate, sinuate, upper angle $45^{\circ}$, lower angle $75^{\circ}$ from the nut, both sides sericeous-velutine; nut 6.5-7 $\times 4.5-5 \mathrm{~mm}$, ovoid to suborbicular, sericeousvelutine; areole 4.5-7 $\times$ 4-5 mm, wide-ovate. Seeds $5-5.5 \times 4-4.2 \mathrm{~mm}$, testa smooth.

Specimens analyzed: BRAZIL. Alagoas: sin. loc., XI-1933, fl., Kuhlmann 16053 (RB); Mun. Água
Branca, 13-IX-1954, fl., Falcão 1070 (RB); loc. cit., 25-VIII-1983, fl., Staviski 649 (MAC, MG); Mun. Palmeira dos Índios, 7-II-1969, fl., Döbereiner 496 (RB). Bahia: sin. loc., s.dat., fl., Bondar 1245 (F). Ceará: sin. loc., s.dat., fl., Loefgren 881 (RB); sin. loc., 6-VII-1984, fr., Ataide 252 (HUEFS, IPA); sin. loc., s.dat., fl., Richa 49 (RB); sin. loc., 1928, fl., Rolland 25 (K); Mun. Acarape, margem da estrada para Garapa, 27-VIII-1994, fr., Silveira s.n. (EAC21293, HUEFS203642); Mun. Alcântaras, sítio Algodões, Serra da Meruoca, 28-II-2000, fl., Fernandes s.n. (EAC29054, HUEFS203641); loc. cit., 5-I-1962, fr., Fernandes s.n. (EAC, HUEFS 203648); loc. cit., 10-XII-1976, fr., Fernandes s.n. (EAC 3060, HUEFS 203644); Mun. Baturité, estrada da Serra do Vicente, 16-V-1980, fl. fr., Martins s.n. (EAC8652, HUEFS203645); loc. cit., Serra do Baturité, perto do açude de Aacarape, 18-VII-1939, fr., Eugenio 1297 (HUEFS, RB); loc. cit., Estação Baturité, beira da estrada nas colinas, 10-IV-1909, fl., Ducke 1960 (HUEFS, RB); loc. cit., 17-XII-1967, fl., Döbereiner 406 (RB); Mun. Caridade, 8-III-2002, fl., Fernandes s.n. (EAC 21394); loc. cit., Campos Belos, estrada de inhuporanga, 8-VII-2008, fr., Menezes 30 (EAC); loc. cit., $12 \mathrm{~km}$ de Campos Belos, 04¹0,9'49', $38^{\circ} 59,7^{\prime} 73^{\prime \prime} W, 250$ m, 17-III-2002, fl. fr., Souza 28690 (ESA, RB); Mun. Caucaia, 17-VI-1985, fl., Nunes s.n. (EAC 13267); loc. cit., 3-II-1996, fl., Castro s.n. (EAC 23955); Mun. Cratéus, Grajal, Serra das Almas, 0506'00''S, 4052'22'”W, 250 m, 4-VI-2002, fl., Araújo 1550 (EAC, HUEFS, JPB); Mun. Cruz, para Campos Belos, 6-V-1909, fl., Ducke 2244 (MG, RB); Mun. Fortaleza, Modubim, Av. Perimetral, 2-VII-1974, fl., Matos s.n. (EAC5431, HUEFS204342); Mun. General Sampaio, Fazenda Natália, 29- IV-2007, fl., Moro 113 (EAC); Mun. Guaiúba, Fazenda Guaiúba, 12-IX-1995, fl., Pereira 1 (EAC); Mun. Itaitinga, Sererau, 10-IV-2003, fl. dos Santos s.n. (EAC32666, HUEFS80820); Mun. Jaguaribe, Fazenda Mulungu, 9-VI-1943, Bezerra s.n. (EAC624, HUEFS203649); Mun. Maranguape, a margem da estrada, Sítio Luís Montenegro, 1-V-1955, fl., Bezerra s.n. (EAC1163, HUEFS); loc. cit., estrada para Pitaguari, 7-III-1996, fl., Castro s.n. (EAC23959); loc. cit., pé da Serra de Aratanha, 13-VII-1955, fl., Ducke 2474 (EAC, HUEFS, IAN); loc. cit., Hotel Pirapora, 15-VIII-1935, fr., Drouet 2261, 2279 (F, GH, NY); loc. cit., Serra de Maranguape, X-1910, fr., Ule 9040 (L, K); Mun. Novo Oriente, 25-X-1997, fl., Fernandes s.n. (EAC25940); Mun. Pacatuba, 10-IV-1991, fl., Bezerra s.n. (EAC 27570); Mun. Pacujá, 15-VI-2007, fl., Andrade 

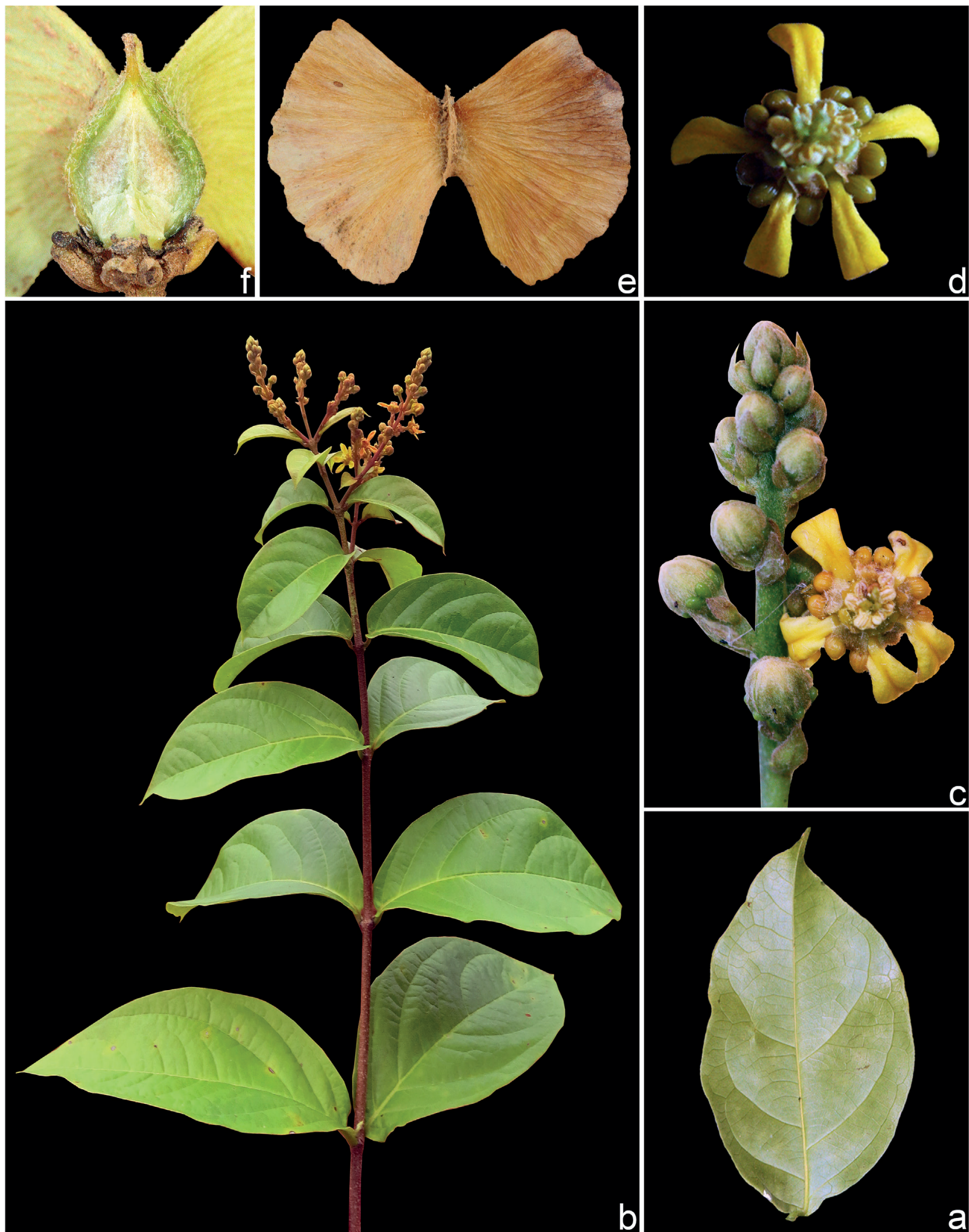

b

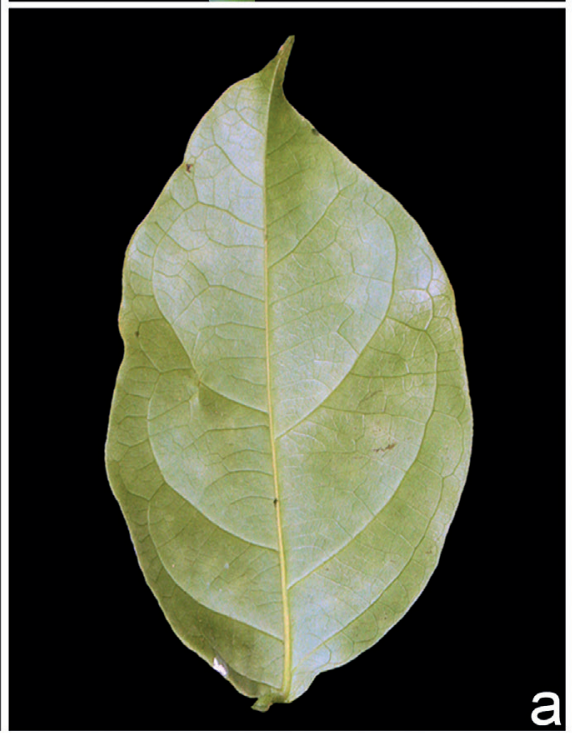

Figure 38. Amorimia septentrionalis W.R. Anderson: a. leaf in abaxial view. b. flowering branch. c. inflorescence. d. flower in frontal view. e. samara in frontal view. f. detail of the areole (photographs a, c-f by M.O.O. Pellegrini; b by M. Alves). 
3098 (HUEFS, HUVA); Mun. Quixadá, 27-VI-1955, fl., Black 55-18227 (EAC, IAN); Mun. Reriutaba, 14-VI-2007, fl., Teixeira 19 (HUEFS, HUVA); Mun. Santa Quitéria, estrada para Itatira a $13 \mathrm{~km}$ da BR-020, 26-IV-1979, fl., Fernandes 5965 (EAC, HUEFS); Mun. São Gonçalo do Amarante, 28-III-1936, fl., Luetzelburg 27022 (K, NY); Mun. Senador Pompeu, XII-1950, fl., Dayton s.n. (IAN 097706); Mun. Sobral, Centro Nacional de Caprinos, 29-VII-1977, fl., Mello s.n. (BAH 2055). Maranhão: Mun. Vargem Grande, 20-V-1933, fl., Luetzelburg 23600 (NY). Paraíba: sin. loc., II-1933, fl., von Ihering s.n. (HUEFS204345, SP30234); Mun. Aguiar, sítio São Bento, areal aguiar, 0706'22,6”'S, 38 $12^{\circ}$ '24, '” W, 273 m, 16-IV-2014, fl., Fontana 8002 (RB); Mun. Alagoa Grande, VII-1922, fl., Gusmão 26277 (RB); Mun. Araruna, entrada do Parque Estadual da Pedra da Boca, 06 26'26's, 35³8'31"'W, 220 m, 16-III-2003, fl. Lima 1763 (HUEFS, JPB); Mun. Areia, 18-X-1958, fr., Moraes s.n. (CEPEC78956, HUEFS, IAN1943, RB104082); Mun. Cajazeiras, Engenheiros Ávidos, Serra de Santa Catarina, IX-2009, fr., Gadelha Neto 2649 (HUEFS, JPB, RB); Mun. Guarabira, Fazenda Boa Sorte, 19-IX-1937, fl., Deslandes 10 (HUEFS, SP); Mun. Juarez Távora, 5-IX-1996, fl., Barbosa 1532 (HUEFS, JPB); Mun. Souza, Fazenda Riacho da Taba, 11-VI1993, fr., Gadelha Neto 32 (HUEFS, JPB); loc. cit., 6-III-1994, fl., Gadelha Neto 82 (HUEFS, JPB); loc. cit., São Gonçalo, 1935, fl. fr., Seccas 54 (HUEFS, RB); loc. cit., 12-VI-1941, fl., Vidal 17730 (HUEFS, IPA); Mun. Tacima, Pedra da Boca, 18-V-2002, fl., Agra 5864 (HUEFS, JPB); Pernambuco: Mun. Betânia, Tapera, 14-VI-1932, fl., Pickel s.n. (NY00476096); loc. cit., VIII-1930, fl., Pickel 26 (F); loc. cit., IX1920, fl., Pickel 278 (IAN, IPA, OUPR); Mun. Bom Conselho, 7-II-1969, fr., Döbereiner 498 (PAMG, RB, MO); Mun. Carpina, Mata do Engenho Trapuá, Usina Petribú, 10-VIII-1996, fl. fr., Lucena 608 (HST, K, PEUFR); Mun. Limoeiro, 18-VI-1973, fl., Foerster s.n. (IAN); loc. cit., Faz. Socorro, 29-VI1950, fl., Leal 192 (HUEFS, RB); loc. cit., 5-XII1972, fl., Döbereiner 398, 399, 400, 401, 402, 403, 404 (RB); Mun. Mirandiba, estrada para Cacimba Nova, 31-III-2006, fl. fr., Pinheiro 94 (CEPEC, UFP); loc. cit., Fazenda Pau de Leite, 17-VII-2008, fr., Pinheiro 997, 998 (JPB, UFP); Mun. Nazaré da Mata, 24-X-1953, fl., Moraes 938 (IAN, UB); Mun. Rio Formoso, 1983, fl., Coelho 47896 (HUEFS, IPA); Mun. Paudalho, 22-XII-1967, fl., Döbereiner 405 (RB); Mun. São Lourenço da Mata, Estação Ecológica do Tapacurá, 10-X-1999, fr., Miranda 3606 (BHCB,
HST); Mun. Serra Talhada, Estação Experimental do IPA, 26-III-1995, fl., Miranda 2215 (HSTA, HUEFS, INPA, UEC); loc. cit., Manga Pedra Branca, 15-V-1968, fl., Lira 68-217 (HUEFS, IPA, OUPR, PAMG); loc. cit., próximo à estação da UFRPE, 12-VII-1990, fr., Bocage 53684 (HUEFS, IPA); Mun. Timbaúba, Fazenda Santa Luzia, 4-IX-1972, fr. Gomes s.n. (IAN140059); Mun. Trindade, 36 km de Vitória, estrada em direção a Ipudi, 10-VII-1994, fl., Pinto 122 (EAC); Mun. Triunfo, na estrada para Princesa Isabel, 25-V-1971, fr., Heringer 925 (HUEFS, IPA, R, RB); Mun. Vicência, Engenho Jundiá, 29-XI1957, fl. fr., Andrade-Lima 2822 (HUEFS, IPA). Piauí: Mun. Conceição do Canindé, Fazenda Matroa, 5-V-1993, fl., Passos s.n. (EAC19709, TEPB6583); loc. cit., Fazenda Porém Enquanto, 5-V-1993, fl., Passos s.n. (EAC19107, TEPB6582); Mun. Elasbão Veloso, Fazenda Santa Maria, 3-V-1993, fl., Passos s.n. (EAC19708, TEPB6581). Rio Grande do Norte: Mun. Açu, Baixa Verde, 18-V-1983, fl. fr., Freitas Filho 182 (UFRN); Mun. Acari, Sítio Talhado, 0619'53'S, 36 37'29'”W, 26-II-2011, fl., Roque 881 (HUEFS, RB, UFRN); Mun. Canguaretama, margem da estrada de acesso à Barra do Cunhaú, 06 ${ }^{\circ} 19^{\prime} 37^{\prime}$ 'S, 3503'29'W, 16-VII-2006, fl. fr., Oliveira 1736 (IPA, MOSS, UFRN); Mun. Macaíba, Escola Agrícola de Jundiaí, Mata do Olho D’água, 26-VIII-2000, fr., Cestaro 47 (UFRN); loc. cit., 17-X-1952, fl., Azevedo 13 (RB); Mun. Mossoró, Sítio Camurupim, 5'11'15"S, 37²0'39'W, 23-IV-2008, fl., Silva s.n. (HUEFS, MOSS, UFRN); Mun. Natal, Macaíba, 16-IX-1951, fl., Alvarenga 5 (PAMG, RB); Mun. Riacho de Santana, Sítio Paul de Cima, 18-VII-1987, fl. fr., Carvalho 4 (HRCB, RB, UB); Mun. Timbaú do Sul, 16-VII-2006, fr., Oliveira 1736 (ASE, HUEFS, MOSS, UFRN). Sergipe: Mun. Maruim, Fazenda Praia, 20-VIII-1971, fr., Döbereiner 790 (ASE, HUEFS, RB); loc. cit., Mata do Caititu, 22-V-2013, fl., Gomes 1094 (ASE); loc. cit., XI-2015, fl. fr., Almeida et al. 800 (CEPEC, HUEFS, RB); loc. cit., XI-2015, fl. fr., Marques 2, 4 (BHCB).

Distribution, habitat and phenology: Amorimia septentrionalis is known from SDTF in the States of Alagoas, Bahia, Ceará, Maranhão, Paraíba, Pernambuco, Piauí, Rio Grande do Norte and Sergipe, Brazil (figure 33). Flowering and fruiting throughout the year.

Conservation status: Amorimia septentrionalis possesses a wide EOO of ca. $499,881.756 \mathrm{~km}^{2}$ and an AOO of ca. $28.000 \mathrm{~km}^{2}$. However, it is endemic to 
the Caatinga domain, which has been greatly modified by human activities in the past few decades. Thus, it should be regarded as Endangered [EN, B1ab(iii,iv,v); C2a(i,ii); D2].

Etymology: the epithet makes reference to its northern distribution within the genus in Eastern Brazil.

Taxonomic notes: two years after describing Mascagnia amazonica (= Amorimia amazonica), Niedenzu (1928) published his Malpighiaceae monograph. The author cited two additional collections for this species, besides the type specimen, from the municipalities of Baturité and Ipú, in the State of Ceará, Brazil. Almost 80 years later, Anderson (2006) published a new species of Amorimia based on a collection from an unknown locality also in the State of Ceará, $A$. septentrionalis. Due to its distribution be restricted to Northeastern Brazil, Anderson compared this new species to Amorimia rigida, a recently untangled species complex from Brazil (Almeida et al. 2016b). Contraty to Anderson's comments, $A$. septentrionalis is not morphologically similar to the $A$. rigida complex, but to $A$. amazonica, as previously stated by Niedenzu (1928). This species belongs to the subg. Uncinae, due to sharing several morphological characters, such as abaxially glabrous sepals, obovate to spatulate petals, base cuneate, claws plane, and styles uncinate at apex. It is morphologically related to A. pubiflora and $A$. tumida due to their pubescent filaments or anthers. However, A. septentrionalis is morphologically more similar to A. pubiflora due to their leaf blades with a pair of glands at base, inflorescences with more than 10 flowers, pedicel thin in fruit, filaments glabrous, styles glabrous near apex, and samaras pale green to ocher. Amorimia septentrionalis can be differentiated by its tomentose and lenticellate stems ( $v s$. velutine without lenticels), leaf blades cuneate or rounded at base, tomentose ( $v s$. cordate or subcordate at base, velutine), and petals adaxially sparsely sericeousvelutine ( $v s$. adaxially glabrous).

2.7. Amorimia tumida R.F.Almeida \& A.C.Marques, Phytotaxa305(3): 180.2017. Holotype:BRAZIL. Rio de Janeiro: Mun. Cardoso Moreira, Serra da Bandeira, Mata Estacional Semidecidual Baixo-Montana, 17-XII-2013, fr., M.N. Coelho \& I.G. Costa 2794 (RB barcode 839289!; isotypes: CEPEC!, HUEFS barcode 70656 !).

Figures 39-40

Woody vines; branches striated, sparsely lenticellate, lenticels brown, sparsely sericeous- velutine to glabrous at age; stipules $0.5-0.6 \mathrm{~mm}$ long, narrowly triangular, sericeous, interpetiolar on branches, epipetiolar on inflorescences, persistent to deciduous. Leaves opposite, reduced in inflorescences; petioles 4.3-6.6 mm long, canaliculate, sparsely sericeous-velutine to glabrous at age, eglandular at apex; leaf blades 4-9.5 $\times 2.1-5.1 \mathrm{~cm}$, plane, not bullate, elliptic, base obtuse, margin slightly revolute in sicco, apex acuminate, adaxially glabrous, abaxially sparsely sericeous-velutine to glabrescent at age, eglandular; midvein adaxially impressed, abaxially prominent, 8-9 pairs of secondary veins, arching $48-55^{\circ}$, opposite to subopposite, both sides impressed, reticulum impressed on both sides. Thyrsi (pseudoracemes), axillary; main axis $8.45-9.75 \mathrm{~cm}$ long, cylindrical, striated, sericeous-velutine; cincinni 6-8, 1-flowered, decussate; reduced leaves absent; bracts 1.3-3.3 × 1-1.6 mm, lanceolate, plane, petiolate, deflexed to the peduncle, 1 pair of marginal glands near base, both sides sericeous-velutine; peduncle $11-20 \times 0.7-1 \mathrm{~mm}$, laterally flattened, sericeousvelutine; bracteoles 1.1-1.8 $\times$ 0.5-0.6 mm, oblong, concave, sessile, subopposite, inserted $2.5-4 \mathrm{~mm}$ below the apex of peduncles, deflexed to the peduncle, eglandular, both sides sericeous-velutine. Flowers diameter unknown; floral buds (fragments) $5.5-8 \times 5-6 \mathrm{~mm}$; pedicels (in fruits) $6-8 \times 1-1.5 \mathrm{~mm}$, cylindrical, tumid, sericeous to glabrescent. Sepals $3.5-4 \times 2.5-3 \mathrm{~mm}$, narrowly oblong, not appressed to the androecium, apex obtuse to rounded, revolute at anthesis, both sides sericeous-velutine; glands greenish turning brown at age, 3-3.1 $\times 1.3-1.5 \mathrm{~mm}$. Petals unknown. Stamens opposite to petals probably longer than those opposite to sepals; filaments $1.8-2 \times 0.5-0.7 \mathrm{~mm}$, connate $1-1.5 \mathrm{~mm}$ long at base, both sides entirely pubescent; anthers probably monorphic pubescent. Ovary unknown, probably each carpel with primordial dorsal and lateral wings, probably sericeous-velutine; styles 3 , cylindrical at base and apex, parallel at base and middle, apex apiculate, entirely sericeous-velutine, anterior style 2-2.1 mm long, posterior styles 2.5-2.8 mm long; stigma lateral, crateriform. Samaras metallic green in vivo and in sicco; dorsal wing 10-15 × 5-6 mm, depressed ovate, margin entire, sinuate, both sides sericeous-velutine; lateral wings $1.6-2.3 \times 1.8-3 \mathrm{~cm}$, flabelliform, margin erose, sinuate, upper angle $15^{\circ}$, lower angle $55^{\circ}$ from the nut, both sides sericeousvelutine; nut 5-6 $\times 5-6 \mathrm{~mm}$, narrowly ovoid, sericeousvelutine; areole $8.6-10 \times 2.6-3.5 \mathrm{~mm}$, narrowly-ovoid. Seeds $6.1-9 \times 3.5-6.5 \mathrm{~mm}$, testa rugose with lateral crests. 
Distribution, habitat and phenology: Amorimia tumida is known only from Semi-deciduous forests in northern Rio de Janeiro State, Brazil (figure 33), fruiting in December.

Conservation status: despite recent efforts on recollecting Amorimia tumida in its type locality on Northern State of Rio de Janeiro, I was unable to locate it in the field. The collector of the type specimen did not record geographic coordinates for this specimen, and little was written in the specimen's label about its habitat and locality. Unfortunately, this species remains represented by a single collection from Semideciduous Forests associated to rocky outcrops within the Atlantic Forest of Serra da Bandeira, Northern
State of Rio de Janeiro. Thus, this species should be regarded as Data Deficient (DD).

Etymology: the epithet makes reference to its tumid pedicels in fruiting, a unique feature in the genus.

Taxonomic notes: Amorimia tumida resembles $A$. andersonii (a member of $A$. subg. Amorimia) on the shape and position of bracts and bracteoles, disposition of sepals at anthesis, color of sepal glands in anthesis, indumenta of filaments, shape of styles, and shape of the dorsal wing of samaras. However, it differs of $A$. andersonii on leaf blade shape and presence of glands at base, fewer secondary veins, veins joining the primary vein in an acute angle, fewer flowers in inflorescences, pedicel tumid in fruits, filaments
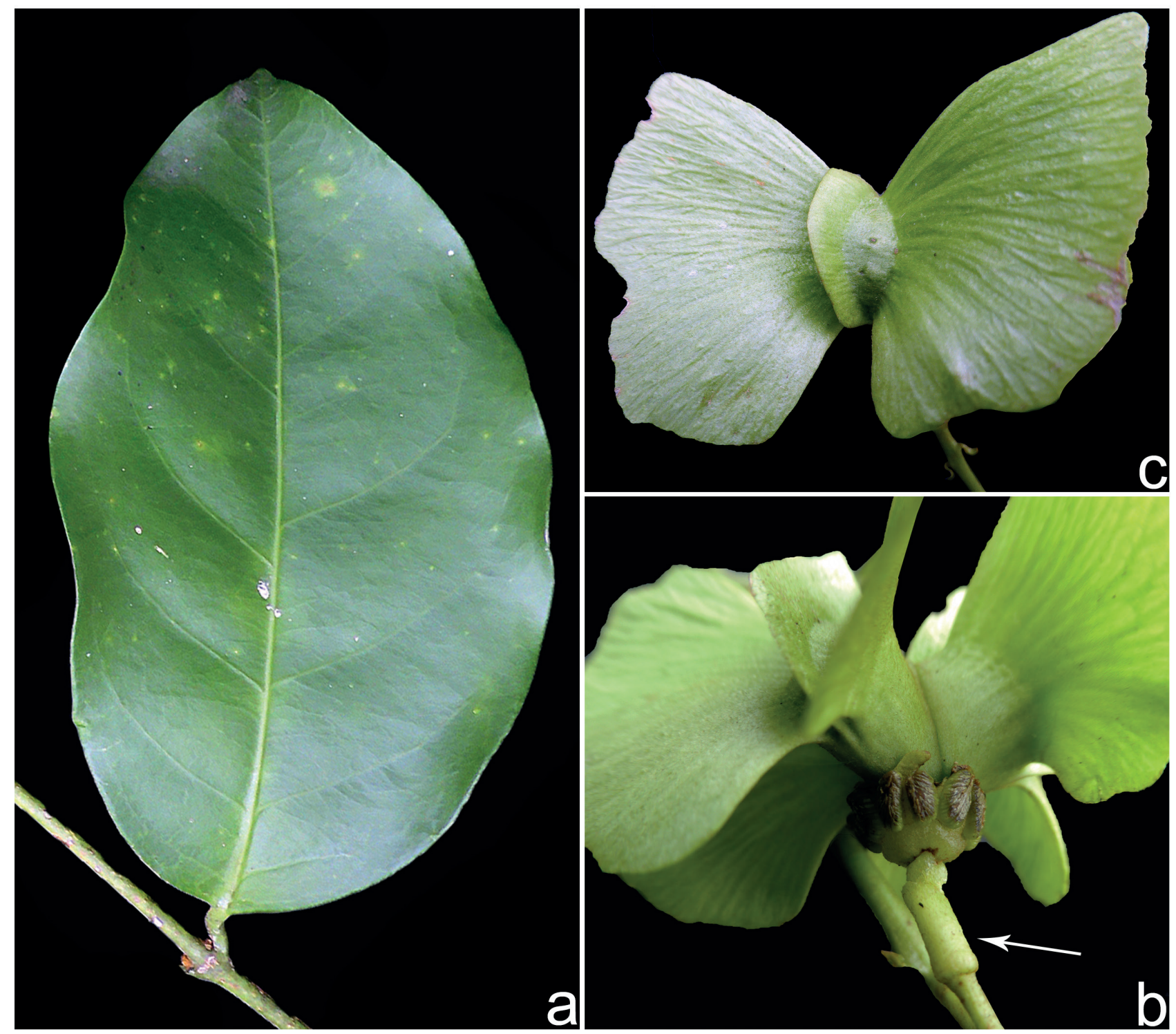

Figure 39. Amorimia tumida R.F. Almeida \& A.C. Marques: a. leaf in adaxial view. b. samara in side view showing the tumid pedicel. c. samara in frontal view (photographs by M.N. Coelho). 

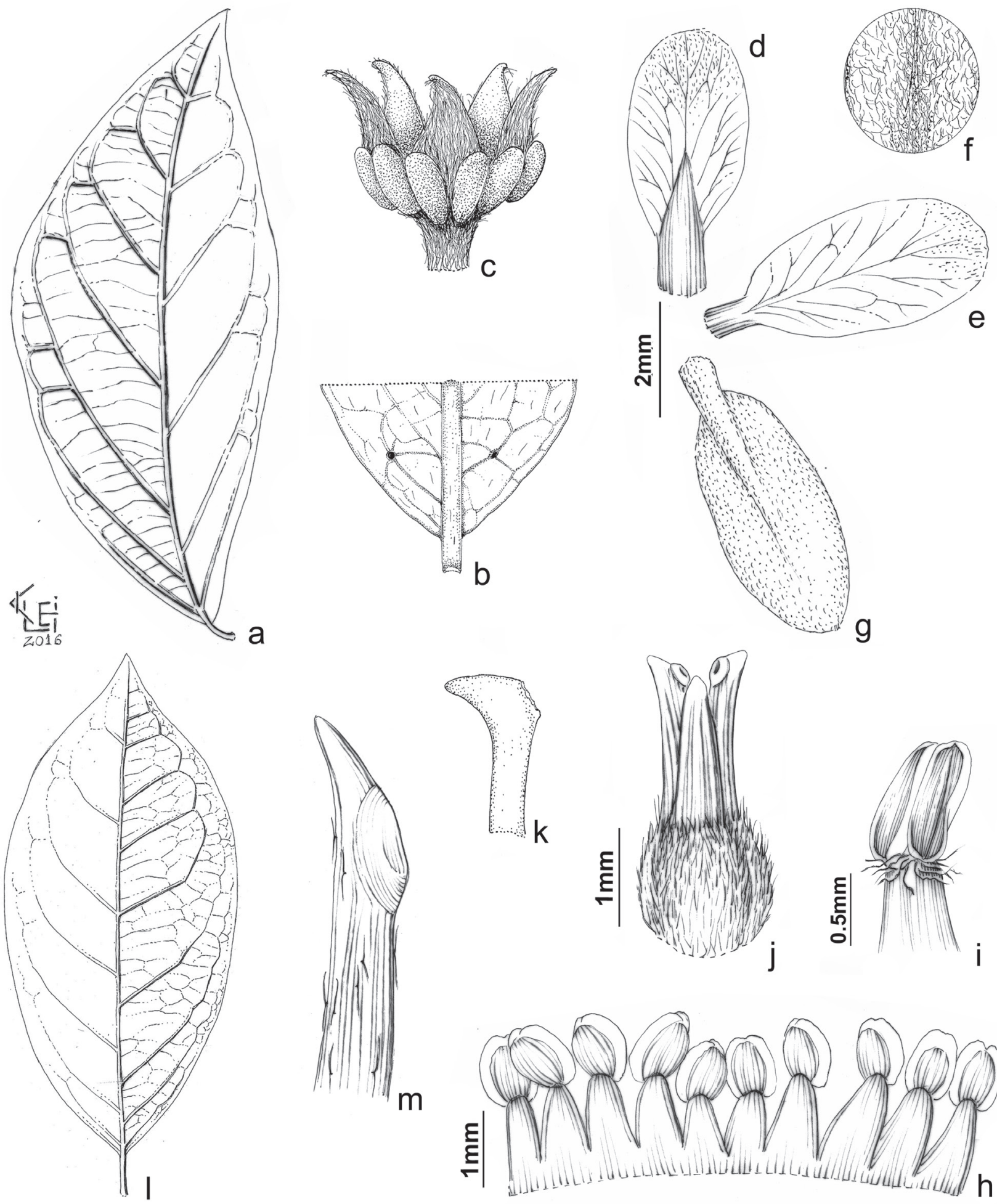

$\mathrm{m}$

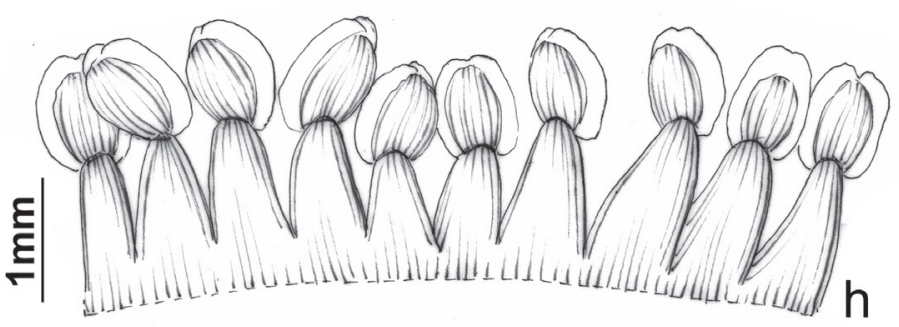

Figure 40. Amorimia septentrionalis W.R. Anderson: a. abaxial side of a leaf evidencing vein pattern. b. detail of the abaxial side of a leaf evidencing a pair of glands. c. sepals. d. adaxial side of a posterior petal. e. adaxial side of a posterio-lateral petal. f. detail of the indumentum on the abaxial side of lateral petals. g. abaxial side of an anterio-lateral petal. h. androecium. i. detail of a stamen evidencing anther hairs. j. gynoecium. k. detail of the uncinate apex of a style (drawings by Klei Sousa and Karin Weishaar Douthit). Amorimia tumida R.F. Almeida \& A.C. Marques: 1. abaxial side of a leaf evidencing vein pattern and a pair of glands. m. uncinate apex of a style (drawings by Klei Sousa). 
completely sericeous, styles completely sericeous, samaras sericeous. Nonetheless, it can be easily differentiated by its leaf blades without a pair of glands at base, inflorescences up to 8-flowered, pedicel thickened in fruit, filaments pubescent, style pubescent near apex, and samaras metallic green to ocher.

\section{Acknowledgements}

I would like to thank Klei Sousa for the drawings; the University of Michigan Herbarium for the partial drawings of $A$. kariniana, A. rigida, and $A$. septentrionalis; the staff and curators of all herbaria (ALCB, AMAZ, ASE, BAH, BM, BHCB, BOTU, CEN, CEPEC, CESJ, CGMS, COL, CPAP, CRVD, CTES, CUZ, EAC, ESA, F, FLOR, FUEL, FURB, FZB, G, GH, GUA, HAS, HB, HCF, HEPH, HRB, HRCB, HSJRP, HST, HUCP, HUCS, HUEFS, HUEM, HUESC, HUFG, HUFU, HUPG, HURB, HUT, IAC, IAN, ICN, INPA, IPA, JPB, L, LIL, K, MAC, MBM, MBML, MG, MICH, MO, MPU, NY, OUPR, P, PACA, PAMG, PEUFR, PMSP, R, RB, RBR, RFA, S, SI, SMF, SP, SPF, SPSF, U, UB, UEC, UFP, UFMS, UFMT, UFRN, UPCB, US, USZ, W, VIC, and VIES) for their assistance and loans; André Marcio Araújo Amorim and Denise Maria Trombert Oliveira for financial support for fieldwork; Marco Octavio de Oliveira Pellegrini for support on fieldwork and valuable comments on an early version of the manuscript; and Christiane Anderson, and an anonymous reviewer, for extensive and valuable comments on the manuscript. RFA was sponsored by a FAPESB Ph.D. fellowship (grant \#BOL0584/2013), and by a Cuatrecasas Award (Smithsonian Institution). Fieldwork was supported by Reflora Malpighiales Project (grant \#563548/2010-0), and Universal Malpighiales Project (grant \#486079/2013-9). This study is part of my Ph.D thesis carried out from 20132017 on the Programa de Pós-Graduação em Botânica from the Universidade Estadual de Feira de Santana, Bahia, Brazil.

\section{Literature cited}

Almeida, R.F. 2017. Systematics and diversification of Amorimia W.R.Anderson (Malpighiaceae). Tese de Doutorado, Universidade Estadual de Feira de Santana, Feira de Santana, Brazil.

Almeida, R.F., Francener, A. \& Amorim, A.M.A. 2016a. A generic synopsis of Malpighiaceae in the Atlantic Forest. Nordic Journal of Botany 34: 285-301.
Almeida, R.F., van den Berg, C. \& Amorim, A.M.A. 2016b. Untangling the Amorimia rigida complex, a puzzling group of lianescent Malpighiaceae from Eastern Brazil. Phytotaxa 284: 1-23.

Almeida, R.F., Mello, A.C.M.P., Oliveira, D.M.T. \& Amorim, A.M.A. 2017a. Leaf anatomy and macromorphology uncover a new species of Amorimia (Malpighiaceae) from Southeastern Brazil. Phytotaxa 305: 179-190.

Almeida, R.F., Amorim, A.M., Corrêa, A.M.S. \& van den Berg, C. 2017b. A new infrageneric classification for Amorimia (Malpighiaceae) based on morphological, phytochemical and molecular evidence. Phytotaxa 313: 231-248.

Anderson, C.E. 1997. Monograph of Stigmaphyllon (Malpighiaceae). Systematic Botany Monographs 51: 1-313.

Anderson, W.R. 1981. Malpighiaceae. In: The botany of the Guayana Highland - Part XI. Memoirs of the New York Botanical Garden 32: 21-305.

Anderson, W.R. 1987. Notes on Neotropical Malpighiaceae - II. Contributions from the University of Michigan Herbarium 16: 55-108.

Anderson, W.R. 2006. Eight segregates from the Neotropical genus Mascagnia (Malpighiaceae). Novon 16: $168-204$.

Anderson, W.R \& Davis, C.C. 2007. Generic adjustments in Neotropical Malpighiaceae. Contributions from the University of Michigan Herbarium 25: 137-166.

Arruda, F.P., Caldeira, F.H.B., Ducatti, K.R., Bezerra, K.S., Marcolongo-Pereira, C., Lee, S.T., Cook, D., Riet-Correa, F., Colodel, E.M. 2017. Experimental poisoning by Niedenzuella stannea in cattle and corresponding detection of monofluoracetate. Ciência Rural 47: e20160761.

Assis, T.S., Medeiros, R.M.T., Araújo, J.A.S., Dantas, A.F.M. \& Riet-Correa, F. 2009. Intoxicações por plantas em ruminantes e equídeos no Sertão Paraibano. Pesquisa Veterinária Brasileira 29: 919-924.

Assis, T.S., Medeiros, R.M.T., Riet-Correa, F., Galiza, G.J.N., Dantas, A.F.M. \& Oliveira, D.M. 2010. Intoxicações por plantas diagnosticadas em ruminantes $\mathrm{e}$ equínos e estimativa das perdas econômicas na Paraíba. Pesquisa Veterinária Brasileira 30: 13-20.

Bachman, S., Moat, J., Hill, A.W., Torre, J. \& Scott, B. 2011. Supporting Red List threat assessments with GeoCAT: geospatial conservation assessment tool. $e$-Infrastructures for data publishing in biodiversity science. ZooKeys 150: 117-126.

Barbosa, R.R., Silva, I.P. \& Soto-Blanco, B. 2008. Development of conditioned taste aversion to Mascagnia rigida in goats. Pesquisa Veterinária Brasileira 28: 571-574. 
Barros, M.A.B. \& Meneguetti, D.U.O. 2014. Relato de prováveis mortes súbitas em bovinos causadas pela ingestão de Amorimia (Mascagnia) sepium (Malpighiaceae) no estado de Rondônia, Brasil. South American Journal of Basic Education, Technical and Technological 1:3-9.

Becker, M., Caldeira, F.H.B., Carneiro, F.M., Oliveira, L.P., Tokarnia, C.H., Riet-Correa, F., Lee, S.T. \& Colodel, E.M. 2013. Importância da intoxicação por Amorimia pubiflora (Malpighiaceae) em bovinos em Mato Grosso: reprodução experimental da intoxicação em ovinos e bovinos. Pesquisa Veterinária Brasileira 33: 1049-1056.

BFG (The Brazilian Flora Group). 2015. Growing knowledge: an overview of Seed Plant diversity in Brazil. Rodriguésia 66: 1085-1113.

Borboleta, L.R., Labarrere, C.R., Ribeiro, A.F.C., Paes-Leme, F.O., Paes, P.R.O., Ocarino, N.M. \& Melo, M.M. 2011. Perfil bioquímico sanguíneo na intoxicação experimental com extrato de Mascagnia rigida (A.Juss.) Griseb. (Malpighiaceae) em coelhos. Arquivos Brasileiros de Medicina Veterinária e Zootecnia 63: 1113-1123.

Borboleta, L.R., Silva, E.F., Oliveira, M.S., Michel, A.F.R.M., Ferraz, V.P., Leite, R.C. \& Melo, M.M. 2012. Cardiac evaluation after experimental intoxication by Amorimia rigida (Malpighiaceae) extracts in rabbits. Journal of Venomoues Animals and Toxins including Tropical Diseases 18: 295-305.

Cameron, K.M., Chase, M.W., Anderson, W.R. \& Hills, H.G. 2001. Molecular Systematics of Malpighiaceae: Evidence from plastid $r b c L$ and matK sequences. American Journal of Botany 88: 1847-1862.

Carvalho, S.L. \& Pitelli, R.A. 1992. Levantamento e análise fitossociológica das principais espécies de plantas daninhas de pastagens da região de Selvíria, Mato Grosso do Sul. Planta Daninha 10: 25-32.

CEPF (Critical Ecosystem Partnership Fund). 2011. The Atlantic Rainforest. Available in http://www.cepf.net/ Pages/default.aspx (access in 15-II-2017).

Davis, C.C., Anderson, W.R. \& Donoghue, M.J. 2001. Phylogeny of Malpighiaceae: Evidence from chloroplast $n d h F$ and trnL-F nucleotide sequences. American Journal of Botany 88: 1830-1846.

Davis, C.C., Bell, C.D., Mathews, S., Donoghue, M.J. 2002. Laurasian migration explains Gondwanan disjunctions: Evidence from Malpighiaceae. Proceedings of the National Academy of Sciences of the United States of America 99: 6833-6837.

Davis, C.C. \& Anderson, W.R. 2010. A complete generic phylogeny of Malpighiaceae inferred from nucleotide sequence data and morphology. American Journal of Botany 97: 2031-2048.

Duarte, A.L., Medeiros, R.M.T \& Riet-Correa, F. 2013. Intoxicação por Amorimia spp. em ruminantes. Ciência Rural 43: 1294-1301.
ESRI-Environmental Systems Research Institute. 2010. ArcGIS, version 9.3.1. Redlands, California: Environmental Systems Research Institute.

Garcez, W.S., Garcez, F.R., Zanella, D.F.P., Hamerski, L., Ferreira, A.G., Barison A. \& Abot, A.R. 2009. Two unusual epicatechin and epiafzelechin derivatives from Mascagnia pubiflora, a plant toxic to cattle. Journal of the Brazilian Chemical Society 20: 674-679.

Gates, B. 1982. Monographs of Banisteriopsis, and Diplopterys (Malpighiaceae). Flora Neotropica 30: 1-238.

Grisebach, A.H.R. 1858. Malpighiaceae. In: C.F.P. Martius. Flora Brasiliensis 12: 1-92.

IUCN (União Internacional para a Conservação da Natureza). 2012. IUCN red list categories and criteria: Version 3.1. Gland, Switzerland and Cambridge, U. K.: IUCN Species Survival Commission.

Johnson, D.M. 1986. Revision of the Neotropical genus Callaeum (Malpighiaceae). Systematic Botany 11: 335-353.

Jussieu, A.H.L. 1833. Malpighiaceae. In: A.F.C.P. SaintHilaire. Flora Brasiliae Meridionalis, 4 ed., 3: 1-14.

Lago, E.P., Melo, M.M., Araújo, R.B., Nascimento, E.F., Silva, E.F. \& Melo, M.B. 2009. Perfis eletrocardiográfico e ecodopplercardiográfico de ovinos após ingestão da suspensão aquosa de Mascagnia rigida Griseb. (Malpighiaceae). Arquivos Brasileiros de Medicina Veterinária e Zootecnia 61: 853-862.

Lee, S.T., Cook, D., Riet-Correa, F., Pfister, J.A., Anderson, W.R., Lima, F.G. \& Gardner, D.R. 2012. Detection of monofluoroacetate in Palicourea and Amorimia species. Toxicon 60: 791-796.

Marchese, C. 2015. Biodiversity hotspots: a shortcut for a more complicated concept. Global Ecology and Conservation 3: 297-309.

McNeill, J., Barrie, F.R., Buck, W.R., Demoulin, V., Greuter, W., Hawksworth, D.L., Herendeen, P.S., Knapp, S., Marhold, K., Prado, J., Prud'Homme Van Reine, W.F., Smith, G.F., Wiersema, J.H. \& Turland, N.J. 2012. International Code of Botanical Nomenclature (Melbourne Code). Adopted by the Eighteenth International Botanical Congress Melbourne, Australia, July 2011. Regnum Vegetabile 154. A.R.G. Gantner.

Medeiros, R.M.T., Neto, S.A.G., Barbosa, R.C., Lima, E.F. \& Riet-Correa, F. 2002. Sudden bovine death from Mascagnia rigida in Northeastern Brazil. Veterinary and Human Toxicology 44: 286-288.

Melo, M.M., Junior, D.V., Pinto, M.C.L., Silveira, J.B., Ferraz, V., Ecco, R. \& Paes P.R.O. 2008. Intoxicação experimental com extratos de Mascagnia rigida (Malpighiaceae) em camundongos. Arquivos Brasileiros de Medicina Veterinária e Zootecnia 60: 631-640.

Mello, A.C.M.P. 2017. Leaf and floral anatomy of Amorimia (Malpighiaceae) and their phylogenetic implications. Masters dissertation. Universidade Federal de Minas Gerais, Belo Horizonte, Brazil. 
Morton, C.V. 1932. Five new South American species of Mascagnia. Proceedings of the Biological Society of Washington 45: 49-54.

Myers, N., Mittermeier, R.A., Mittermeier, C.G., da Fonseca, G.A.B., Kent, J. 2000. Biodiversity hotspots for conservation priorities. Nature 403: 853-858.

Neto, S.A.G., Sakamoto, S.M. \& Soto-Blanco, B. 2013. Inquérito epidemiológico sobre plantas tóxicas das mesoregiões Central e Oeste do Rio Grande do Norte. Ciência Rural 43: 1281-1287.

Niedenzu, F. 1914. Malpighiaceae americanae III. Arbeiten aus dem botanischen Institut des Kgl. Lyceum hosianum in Braunsberg, pp. 1-61.

Niedenzu, F. 1926. Malpighiaceae novae. Arbeiten aus dem botanischen Institut der Staatlichen Akademie (vorm. Kgl. Lyceum hosianum) in Braunsberg, pp. 59-64.

Niedenzu, F. 1928. Malpighiaceae. In: A. Engler (ed.). Das Pflanzenreich IV 141: 1-870.

Nogueira, V.A., Peixoto, T.C., França, T.N., Caldas, S.A. \& Peixoto, P.V. 2011. Intoxicação por monofluoracetato em animais. Pesquisa Veterinária Brasileira 31: 823-838.

Pavarini, S.P., Soares, M.P., Bandarra, P.M., Gomes, D.C., Bandinelli, M.B., Cruz, C.E.F. \& Driemeier, D. 2011. Mortes súbitas em bovinos causadas por Amorimia exotropica (Malpighiaceae) no Rio Grande do Sul. Pesquisa Veterinária Brasileira 31: 291-296.

Pessoa, C.R.M., Medeiros, R.M.T. \& Riet-Correa, F. 2013. Importância econômica, epidemiologia e controle das intoxicações por plantas no Brasil. Pesquisa Veterinária Brasileira 33: 752-758.

Radford, A.E., Dickison, W.C., Massey, J.R. \& Bell, C.R. 1974. Vascular Plant Systematics. Harper \& Row, New York.

Särkinen, T., Iganci, J.R.V., Linares-Palomino, R., Simon, M.F. \& Prado, D.E. 2011. Forgotten forests issues and prospects in biome mapping using Seasonally Dry Tropical Forests as a case study. BMC Ecology 11: 1-27.

Schons, S.V., Mello, T.L., Riet-Correa, F. \& Schild, A.L. 2011. Poisoning by Amorimia (Mascagnia) sepium in sheep in northern Brazil. Toxicon 57: 781-786.

Silva, D.M., Riet-Correa, F., Medeiros, R.M.T. \& Oliveira, O.F. 2006. Plantas tóxicas para ruminantes e equídeos no Seridó Ocidental e Oriental do Rio Grande do Norte. Pesquisa Veterinária Brasileira 26: 223-236.

Silva, I.P., Lira, R.A., Barbosa, R.R., Batista, J.S. \& Soto-Blanco, B. 2008. Intoxicação natural pelas folhas de Mascagnia rigida (Malpighiaceae) em ovinos. Arquivos do Instituto Biológico 75: 229-233.

Soares, M.P., Pavarini, S.P., Adrien, M.L., Quevedo, P.S., Schild, A.L., Peixoto, P.V., Cruz, C.E.F. \& Driemeier, D. 2011. Amorimia exotropica poisoning as a presumptive cause of myocardial fibrosis in cattle. Journal of Veterinary Diagnostic Investigation 23: $1226-1229$.
speciesLink. 2017. Rede de coleções speciesLink. Available in http://www.splink.org.br/index?lang=pt (access in 15-II-2017).

Spjut, R.W. 1994. A systematic treatment of fruit types. The New York Botanical Garden, New York.

Stafleu, F.A. \& Cowan, R.S. 1986. Taxonomic literature. A selective guide to botanical publications and collections with dates, commentaries and types, 2 ed., vol. 6 . Regnum Vegetabile 115. A.R.G. Gantner Verlag, Rugell.

Thiers, B. 2017 (and continuously updated). Index Herbariorum: A global directory of public herbaria and associated staff. New York Botanical Garden's Virtual Herbarium. Available in http://sweetgum.nybg.org/ih/ (access in 15-II-2017).

Tokarnia, C.H., Canella, C. \& Döbereiner, J. 1961. Intoxicação por um "tinguí” (Mascagnia rigida Griseb.) em bovinos no Nordeste do Brasil. Arquivos do Instituto de Biologia Animal 4: 203-215.

Tokarnia, C.H. \& Döbereiner, J. 1973. Intoxicação por Mascagnia pubiflora em bovinos no Estado de Mato Grosso. Pesquisa Agropecuária Brasileira 8: 61-68.

Tokarnia, C.H. \& Döbereiner, J. 1985. Intoxicação por Mascagnia aff. rigida (Malpighiaceae) em bovinos no norte do Estado do Espírito Santo. Pesquisa Veterinária Brasileira 5: 77-91.

Tokarnia, C.H., Peixoto, P.V. \& Döbereiner, J. 1990. Poisonous plants affecting heart function of cattle in Brazil. Brazil. Pesquisa Veterinária Brasileira 5: 77-91.

Tokarnia, C.H., Peixoto, P.V. \& Döbereiner, J. 1994. Aspectos clínicopatológicos complementares da intoxicação por algumas plantas tóxicas brasileiras. Pesquisa Veterinária Brasileira 14: 111-122.

Tokarnia, C.H., Armién, A.G., Peixoto, P.V., Barbosa, J.D., Brito, M.F. \& Döbereiner, J. 1996. Estudo experimental sobre a toxidez de algumas plantas ornamentais em bovinos. Pesquisa Veterinária Brasileira 16: 5-20.

Tokarnia, C.H., Döbereiner, J. \& Peixoto, P.V. 2000. Plantas Tóxicas do Brasil. Helianthus, Rio de Janeiro.

Tokarnia, C.H., Döbereiner, J. \& Peixoto, P.V. 2002. Poisonous plants affecting livestock in Brazil. Toxicon 40: 1635-1660.

Vasconcelos, J.S., Riet-Correa, F., Dantas, A.F.M., Medeiros, R.M.T., Galiza, G.J.N., Oliveira, D.M. \& Pessoa, A.F.A. 2008. Intoxicação por Mascagnia rigida (Malpighiaceae) em ovinos e caprinos. Pesquisa Veterinária Brasileira 28: 521-526.

Weberling, F. 1965. Typology of inflorescences. Botanical Journal of the Linnean Society 59: 15-221.

Weberling, F. 1989. Morphology of flowers and inflorescences. Cambridge University, Cambridge, $348 \mathrm{pp}$.

WWF (World Wildlife Fund). 2017. Tropical and subtropical dry broadleaf forests. Available in http://www.worldwildlife.org/biomes/tropical-andsubtropical-dry-broadleaf-forests (access in 15-II-2017). 


\section{Collectors' list}

S.leg 1545 KH 2013/0134821 (2.5.); Academia Brasileira de Ciências 780 (1.6.); Acevedo-Rodríguez 14780 (2.1.); Agra 5864 (2.6.); Almeida 800 (2.6.), 549 (1.4.), 561 (1.7.), 562 (1.7.), 594 (1.2.), 614 (1.6.), 615 (1.3.); Alvarenga 5 (2.6.); Amorim 319 (1.5.), 2483 (1.5.), 2772 (1.8.), 3565 (1.7.), 3801 (1.6.), 4102 (1.5.), 4127 (1.5.), 6856(1.1.), 7425 (1.5.), 7426(1.5.), 7512 (1.5.), 7525 (1.5.), 7526 (1.5.), 7528a(1.5.), 7529 (1.5.); Andrade 3098 (2.6.); Andrade-Lima 2822 (2.6.), 7664 (1.6.); Andreata 373 (1.3.), 708 (1.3.); Aona 3852 (1.8.), 3892 (1.8.), 4208 (1.6.); Arantes s.n. HUEFS 211196 (2.5.), s.n. HUEFS 211197 (2.5.), s.n. HUFU 28411 (2.5.); Araújo 838 (1.7.), 1550 (2.6.); Arbocz 656 (2.5.); Arteta s.n. COL 50614 (2.3.); Asplund 16617 (2.4.), 17586 (2.4.); Assis 1522 (1.5.); Ataíde 252 (2.6.); Azevedo 13 (2.6.); Barbosa 1532 (2.6.); Barbour 4256 (2.1.); Barros 160 (1.5.), 4909 (1.3.); Barroso s.n. ALCB 1930 (1.2.), s.n. ALCB 1937 (1.2.); Bastos 266 (1.2.), 420 (1.6.), 818 (1.2.); Batatinha 01 (1.6.); Bautista 780 (1.6.); Belém 3551 (1.5.); Bezerra s.n. EAC 624 (2.6.), s.n. EAC 1163, s.n. EAC 27570 (2.6.), s.n. HUEFS 203649 (2.6.); Bicudo 88 (2.5.); Black 55-18227 (2.6.); Blanchet 1660 (1.5.), 1720 (1.5.), s.n. P 04843525 (1.5.); Bocage 53684 (2.6.); Bondar 1245 (2.6.); Botero s.n. COL 52205 (2.3.); Britez s.n. UPCB 32213 (1.4.); Brito 23 (1.2.), 28 (1.2.); Brozec 12 (1.7.); Buttura 567 (1.4.); Caliente 609 (2.5.); Campos 2015 (2.2.), 2490 (2.2.), 2658 (2.2.), 3266 (2.2.), 4846 (2.2.); Cardoso 40 (1.6.), 493 (1.6.), 518 (1.6.); Carvalho 4 (2.6.), 29 (1.6.), 111 (1.6.), 114 (1.6.), 296 (1.6.); Carvalho-Sobrinho 2353 (1.6.); Castañeda 9252 (2.3.), 9292 (2.3.), 9646 (2.3.), 10040 (2.3.); Castro 1193 (1.8.), 1281 (1.6.), s.n. EAC 23955 (2.6.), s.n. EAC 23959 (2.6.); Caxambu 6011 (1.4.); 7035 (1.4.); Cervi 8134 (1.4.); Cestaro 47 (2.6.); Coelho 2794 (2.7.), 47896 (2.6.); Corbett s.n. HUEFS 204337 (2.5.), s.n. SP 45967 (2.5.); Claussen s.n. P 4843527 (1.7.); Cordeiro 1217 (1.4.), 1997 (1.4.), 2678 (1.8.); Costa 271 (2.1.); 363 (2.5.), 1630 (1.2.), 1998 (1.2.), 2006 (1.2.), 2124 (1.2.); Couto 35 (1.6.), 1130 (1.5.); Croat 51085 (2.1.); Cruz 32 (1.6.); Cunha 741 (1.7.); Daly 9637 (2.1.), 13263 (2.1.); Dantas 104 (1.3.), 176 (1.3.), 283 (1.3.), 610 (1.3.); Dawson 15006 (1.8.); Dayton s.n. IAN 097706 (2.6.); Demuner 1101 (1.5.); Deslandes 10 (2.6.); Döbereiner 398 (2.6.), 399 (2.6.), 400 (2.6.), 401 (2.6.), 402 (2.6.), 403 (2.6.), 404 (2.6.), 405 (2.6.), 406 (2.6.), 496 (2.6.), 498 (1.6.), 538 (2.6.), 774 (2.5.), 790 (2.6.), 795 (2.5.), 803 (2.5.), 858 (1.7.),
901 (2.5.), 1673 (1.7.), 1677 (1.5.), 1765 (1.5.), 1771 (1.5.), 1773 (1.5.), 1774 (1.5.), s.n. NY 01018898 (1.5.), s.n. NY 01018899 (1.5.); Drouet 2261 (2.6.), 2279 (2.6.); Ducke 1960 (2.6.), 2244 (2.6.), 2474 (2.6.); Durigon 570 (1.4.); Dusén 14093 (1.4.); Emrich s.n. PACA 26863 (1.4.), s.n. PACA 30022 (1.4.) s.n. PACA 32867 (1.4.), s.n. PACA 52618 (1.4.); Encarnación 26053 (2.1.), 26055 (2.1.); Espinosa 901 (1.5.); Eugenio 1297 (2.6.); Fagerlind 242 (2.4.); Falcão 1070 (2.6.); Farag 309 (1.3.); Farfán 779 (2.1.); Farney 3963 (1.3.), 4650 (1.3.); Fernandes 403 (1.3.), 5965 (2.6.), s.n. EAC 3060 (2.6.), s.n. EAC 21394 (2.6.), s.n. EAC 25940 (2.6.), s.n. EAC 29054 (2.6.), s.n. HUEFS 203641 (2.6.), s.n. HUEFS 203644 (2.6.), s.n. HUEFS 203648 (2.6.); Ferreira 233 (1.2.), 339 (1.6.), s.n. PAMG 11405 (2.5.); Ferreyra 4744 (2.2.); Fiaschi 2761 (1.7.); Foester s.n. IAN; Fontana 8002 (2.6.); Forzza 5534 (1.5.); Foster 11965 (2.1.); Fox 19 (1.4.); Francisco 11 (1.7.); Freitas s.n. JPB 54378 (1.7.), s.n. VIC 26216 (1.7.); Freitas Filho 182 (2.6.); Gadelha Neto 32 (2.6.), 82 (2.6.), 2649 (2.6.); Garcez s.n. HUEFS 207597 (2.5.); Gardner 5394 (1.3.); Gatto 01 (1.6.); Gentry 26915 (2.1.), 37660 (2.1.), 44992 (2.1.); Glaziou 12493 (1.7.), 13601 (1.7.), 20757 (2.5.); Gomes 1094 (2.6.), s.n. IAN 140059 (2.6.); Gottsberger 24-25173 (1.7.); Graham 205 (2.1.), 866 (2.1.); Grings 752 (1.4.), 1186 (1.4.), 1774 (1.4.); Groppo 983 (1.5.); Grupo Pedra do Cavalo 8 (1.6.), 1021 (1.6.), 1031 (1.6.); Guaglianone 2128 (1.4.); Guedes 7291 (1.6.), 12067 (1.6.), 13101 (1.6.), 16327 (1.7.), 18404 (1.5.), 23072 (1.8.); Gusmão 26277 (2.6.); Hagelund 3210 (1.4.); Harling 3026 (2.4.); Hatschbach 3780 (1.4.), 18685 (1.4.), 20871 (1.4.), 21887 (2.5.), 37957 (1.4.), 42983 (1.4.); Haught 3070 (2.4.), 3927 (2.3.); Hayward s.n. (1.4.); Heringer 462 (1.6.), 925 (2.6.); Hilaire 1501 (1.7.), s.n. P barcode 02429221 (2.5.); Irgang 384 (1.4.); Klug 4259 (2.2.); Knapp 7575 (2.1.); Kollmann 3726 (1.5.); Krapovickas 39391 (1.4.), 39599 (1.4.), 44714 (1.4.); Krieger 10298 (1.3.); Kuhlmann 3534 (1.3.), 16053 (2.6.); Irwin 17573 (2.5.); Jaccoud 68 (2.5.); Jacques 357 (2.5.); Jardim 4228 (1.3.), 5350 (1.5.); Jesus 1299 (1.6.), 1745 (2.5.); Jost 495 (1.8.); Lara s.n. HUEFS 204344 (2.5.); Leal 192 (2.6.), 246 (1.8.); Lewis 1971 (1.8.); Lima 154 (1.5.), 163 (1.8.), 1763 (2.6.), 2877 (1.3.); Linneo 873 (2.1.); Lira 68-217 (2.6.); Loefgren 881 (2.6.); Lopes 655 (1.7.), 698 (1.5.), 1340 (1.8.); Lordelo 56307 (1.6.); Lowrie 259 (2.1.); Lucena 608 (2.6.); Luetzelburg 23600 (2.6.), 27022 (2.6.); Macedo 2112 (1.8.); Malheiros s.n. HUEFS 204343 (2.5.), s.n. SP 39746 (2.5.); Marchett 155 (1.4.); Marinho 31 (1.8.), 
396 (1.8.), 654 (1.1.), 676 (1.1.); Marino 203 (2.5.); Marques 2 (2.6.), 4 (2.6.), 5 (1.5.), 6 (1.8.), 7 (1.7.), 13 (1.2.), 14 (1.2.), 15 (1.6.), 16 (1.6.); Martinelli 2232 (1.5.), 2240 (1.5.); Martins s.n. EAC 8652 (2.6.), HUEFS 203645 (2.6.); Matos 84 (1.6.), 325 (1.6.), s.n. EAC 5431 (2.6.), s.n. HUEFS 204342 (2.6.); Mattos 22457 (1.4.), 22640 (1.4.); Mattos-Silva 3136 (1.5.), 5117 (1.7.); Mauad 128 (1.5.); Melichenko s.n. IAC 23057 (2.5.); Mello s.n. BAH 2055 (2.6.); Melo 1334 (1.7.), 3936 (1.2.), 4790 (1.6.), 6370 (1.6.), 6372 (1.6.), 8557 (1.2.), 8563 (1.2.); Mendes s.n. HUEFS 204340 (2.5.), s.n. HUEFS 204341 (2.5.); Menezes 30 (2.6.); Mentz s.n. ICN 95118 (1.4.); Miranda 2215 (2.6.), 3606 (2.6.), 6171 (1.6.); Molleta s.n. UFMT (2.5.); Mojica 1 (2.3.); Moraes 938 (2.6.), s.n. CEPEC 78956 (2.6.), IAN 1943 (2.6.), RB 104082 (2.6.); Mori 14435 (1.6.); Moro 113 (2.6.); Moura 3 (1.2.), s.n. HUEFS 69135 (1.6.), s.n. HUEFS 69137 (1.6.); Nascimento 38 (1.6.), 83 (1.6.); Neves 89 (1.6.); Nicolau 1482 (1.4.); Noblick 1839 (1.6.), 3876 (1.6.), 4228 (1.6.); Nunes 266 (1.6.), 1218 (1.6.), s.n. EAC 13267 (2.6.), s.n. RB 571 (1.6.); Oliveira 299 (1.6.), 658 (1.6.), 751 (1.5.), 1553 (1.6.), 1554 (1.6.), 1611 (1.8.), 1736 (2.6.); Owlee 1109 (2.4.); Pace 350 (1.5.); Paixão 676 (1.7.); Parreira 01 (2.5.); Passos s.n. EAC 19107 (2.6.), s.n. EAC 19708 (2.6.), s.n. EAC 19709 (2.6.), s.n. TEPB 6581 (2.6.), s.n. TEPB 6582 (2.6.), s.n. TEPB 6583 (2.6.); Pennell 4033 (2.3.); Pereira 1 (2.6.), 37-84, s.n. UFMT 43014 (2.5.); Pickel 26 (2.6.), 278 (2.6.), s.n. NY 00476096 (2.6.); Pinheiro 94 (2.6.), 997 (2.6.), 998 (2.6.), 1132 (1.7.); Pinto 05-1980 (1.2.), 122 (2.6.), s.n. ALCB 1929 (1.2.), s.n. ALCB 17936 (1.2.); Pirani 5462 (1.8.), s.n. HUEFS 203426 (1.8.); Pohl 5778 (2.5.), 5780 (1.3.), s.n. F 872521 (2.5.), Pott 4204 (2.5.), 10411 (2.5.), 13270 (2.5.); Queiroz 54 (1.6.), 3259 (1.8.), 4539 (1.8.), 4590 (1.8.), 4701 (1.8.), 10607 (1.6.), 10655 (1.6.); Rambo 39952 (1.4.), 41303 (1.4.), 52768 (1.4.); Reis s.n. CEPEC 99176 (1.5.); Rezende 3556 (2.5.); Ribas 8101 (1.4.); Ribeiro s.n. IAC 18036 (2.5.); Richa 49 (2.6.); Riedel 503 (1.5.); Ritter s.n. F 2111927 (1.4.); Rocha-Silva s.n. SP
012033 (2.5.); Rodrigo s.n. JPB 54379 (1.7.), s.n. SP 337133 (1.7.), VIC 23366 (1.7.); Rodrigues-Silva 335 (1.7.); Rojas 753 (2.1.); Rolland 25 (2.6.); Roque 881 (2.6.); Rubio 2445 (2.4.); Rusby 512 (2.1.); Saddi 865 (1.7.); Salas 1 (2.3.), 418 (2.2.); Salino 3703 (2.5.), 3953 (1.7.), 4658 (1.8.), 4713 (1.8.); Salzmann s.n. G barcode 00352762 (1.5.); Sampaio 3219 (1.5.); Santana 542 (1.6.); Santos 21 (1.5.), 354 (1.7.), s.n. EAC 32666 (2.6.), s.n. HUEFS 64423 (1.2.), s.n. HUEFS 80820 (2.6.); SAP 243 (1.8.); Schenkel s.n. ICN 95120 (1.4.); Schlindwein 532 (1.4.), 534 (1.4.); Schuch 5721 (1.3.); Schultz 61 (1.4.), 463 (1.4.); Schwacke 3008 (2.1.), 12939 (1.4.); Schwarz s.n. W 68981 (2.1.); Seccas 54 (2.6.); Sehnem 1390 (1.4.); Seibert 2171 (2.1.); Senna 135 (1.4.); Shepherd 4409 (2.4.), Silva 85 (1.6.), s.n. HST 17938 (1.6.), s.n. HUEFS (2.6.), s.n. IAC 18641 (2.5.), s.n. MOSS (2.6.), s.n. UFRN (2.6.); Silva-Filho s.n. MPUC 17129 (1.4.); Silveira s.n. EAC 21293 (2.6.), s.n. HUEFS 203642 (2.6.); Siqueira 561 (1.5.); Sobral s.n. MBM 73963 (1.4.); Solomon 6515 (2.1.); Souza 5155 (1.8.), 5254 (1.8.), 26565 (1.6.), 28690 (2.6.), s.n. BAH 509 (1.7.); SouzaSilva 15 (1.6.), 20 (1.6.), 612 (1.6.), 650 (1.6.); Spruce 4227 (2.1.); Stannard 5312 (1.8.); Staviski 649 (2.6.); Steinbach 339 (2.1.), 6606 (2.1.); Sucre 1421 (1.3.); Teixeira 19 (2.6.); Thomas 11954 (1.7.), 13476 (1.7.); Tiepolo 632 (1.4.); Timaná 2427 (2.1.); Toledo s.n. IAC 18708 (2.5.); Torrend s.n. ALCB 1928 (1.6.), HUEFS 204347 (1.6.); Trieder 33074 (1.4.); Ule 9040 (2.6.), 9478 (2.1.); Uribe s.n. COL 423253 (2.3.); Valenzuela 1062 (2.1.); Vandeman 3309 (2.1.); Vanilda 146 (1.6.); Vanni 2903 (1.4.); Vásquez 25843 (2.1.); Viana 1397 (1.6.); Vianna s.n. ICN 9660 (1.4.), s.n. U 1367689 (1.4.); Vidal 17730 (2.6.); Vidal-Senege s.n. P 06173021 (2.1.); Vieira 1401 (1.5.), s.n. HUEFS 203425 (2.5.), RB 315272 (2.5.), SPF 23655 (2.5.); Vinha 1204 (1.5.); von Ihering s.n. HUEFS 204345 (2.6.), s.n. SP 30234 (2.6.); Waechter 1176 (1.4.); Warming 838 (1.7.); Wasum 914 (1.4.); Weigend 5735 (2.1.); Woytkowski 5459 (2.1.); 7183 (2.2.), 7200 (2.2.), 7202 (2.2.). 